\title{
Experimental spinal cord stimulation in neuropathic pain models
}

Citation for published version (APA):

Truin, M. (2011). Experimental spinal cord stimulation in neuropathic pain models. [Doctoral Thesis, Maastricht University]. Maastricht University. https://doi.org/10.26481/dis.20110630mt

Document status and date:

Published: 01/01/2011

DOI:

10.26481/dis.20110630mt

Document Version:

Publisher's PDF, also known as Version of record

\section{Please check the document version of this publication:}

- A submitted manuscript is the version of the article upon submission and before peer-review. There can be important differences between the submitted version and the official published version of record.

People interested in the research are advised to contact the author for the final version of the publication, or visit the DOI to the publisher's website.

- The final author version and the galley proof are versions of the publication after peer review.

- The final published version features the final layout of the paper including the volume, issue and page numbers.

Link to publication

\footnotetext{
General rights rights.

- You may freely distribute the URL identifying the publication in the public portal. please follow below link for the End User Agreement:

www.umlib.nl/taverne-license

Take down policy

If you believe that this document breaches copyright please contact us at:

repository@maastrichtuniversity.nl

providing details and we will investigate your claim.
}

Copyright and moral rights for the publications made accessible in the public portal are retained by the authors and/or other copyright owners and it is a condition of accessing publications that users recognise and abide by the legal requirements associated with these

- Users may download and print one copy of any publication from the public portal for the purpose of private study or research.

- You may not further distribute the material or use it for any profit-making activity or commercial gain

If the publication is distributed under the terms of Article $25 \mathrm{fa}$ of the Dutch Copyright Act, indicated by the "Taverne" license above, 


\section{Experimental Spinal Cord Stimulation In \\ Neuropathic Pain Models}




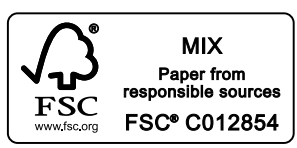

C 2011 Michiel Truin, Maastricht

ISBN: 978-94-6108-181-0

Printed by: Gildeprint Drukkerijen - Enschede

Cover design: Michiel Truin en Janneke Grutters 


\title{
Experimental Spinal Cord Stimulation in Neuropathic Pain Models
}

\author{
PROEFSCHRIFT
}

Ter verkrijging van de graad van doctor aan de Universiteit Maastricht, op gezag van de Rector Magnificus, Prof. Mr. G.P.M.F. Mols, volgens het besluit van het College van Decanen, in het openbaar te verdedigen op donderdag 30 juni 2011 om 14.00 uur

door

Michiel Truin 


\section{Promotor}

Prof. dr. M. van Kleef

\section{Copromotor}

Dr. E.A.J Joosten

\section{Beoordelingscommissie}

Prof. dr. K. van Overbeeke (voorzitter)

Prof. dr. M. de Baets

Dr. R.T.M. van Dongen

Dr. C. Faber

Prof. dr. B. van Hilten

The studies in this thesis were performed within TREND (Trauma Related Neuronal Dysfunction), a knowledge consortium that integrates research on CRPS type 1, and is supported by a Dutch government grant (BSIK03016). 


\section{Contents}

$\begin{array}{ll}\text { Abbreviations } & 7\end{array}$

$\begin{array}{lll}\text { Chapter } 1 \text { General introduction } & 11\end{array}$

$\begin{array}{lll}\text { Part I Mouse models } & 27\end{array}$

Chapter 2 Sensitized uninjured primary afferent related behavioral 29 changes in mice with following motor fiber injury.

Chapter 3 Spinal Cord Stimulation in a mouse chronic neuropathic pain model

Chapter 4 The effect of Spinal Cord Stimulation in mice with chronic neuropathic pain after partial ligation of the sciatic nerve

Part II Improving Spinal Cord Stimulation 67

Chapter 5 Increased efficacy of early Spinal Cord Stimulation in an animal model of neuropathic pain

Chapter 6 The successful pain relief in non-responders to Spinal Cord Stimulation: The combined use of ketamine and Spinal Cord Stimulation

$\begin{array}{lll}\text { Chapter } 7 & \text { General discussion } & 97\end{array}$

$\begin{array}{ll}\text { Bibliography } & 109\end{array}$

$\begin{array}{ll}\text { Summary } & 135\end{array}$

$\begin{array}{lr}\text { Samenvatting } & 139\end{array}$

Co-authors \& affiliations $\quad 143$

$\begin{array}{ll}\text { Dankwoord } & 147\end{array}$

$\begin{array}{ll}\text { Curriculum Vitae } & 153\end{array}$

$\begin{array}{ll}\text { Publications } & 157\end{array}$ 

Abbreviations 



\section{Abbreviations}

$\begin{array}{ll}\text { ANOVA } & \text { Analysis of Variance } \\ \text { AMPA } & \text { a-Amino-3-hydroxy-5-Methyl-4-isoxazolePropionic Acid } \\ \text { CCI } & \text { Chronic Constriction Injury } \\ \text { CGRP } & \text { Calcitonin Gene-Related Protein } \\ \text { CNS } & \text { Central Nervous System } \\ \text { CPIP } & \text { Chronic Post Ischemia Pain } \\ \text { CRPS } & \text { Complex Regional Pain Syndrome } \\ \text { DLF } & \text { DorsoLateral Funiculus } \\ \text { DPO } & \text { Days Post Operative } \\ \text { DRG } & \text { Dorsal Root Ganglion } \\ \text { DRT } & \text { Dorsal Root Transection } \\ \text { EA } & \text { Electro-Acupuncture } \\ \text { EAA } & \text { Excitatory Amino Acid } \\ \text { FBSS } & \text { Failed Back Surgery Syndrome } \\ \text { GABA } & \text { Gamma-Amino Butyric Acid } \\ \text { Glu } & \text { Glutamate } \\ \text { HLA } & \text { Human Leukocyte Antigen } \\ \text { IAA } & \text { Inhibitory Amino Acid } \\ \text { IASP } & \text { International Association for the Study of Pain } \\ \text { i.t. } & \text { intrathecal } \\ \text { LTD } & \text { Long Term Depression } \\ \text { LTP } & \text { Long Term Potentiation } \\ \text { MAPK } & \text { Mitogen-Activated Protein Kinase } \\ \text { MHC } & \text { Major Histocompatability Complex } \\ \text { MPE } & \text { Maximum Possible Effect } \\ \text { NMDA } & \text { N-methyl D-aspartate } \\ \text { NS } & \text { Nociceptive Specific } \\ \text { PCP } & \text { Phenylcycline } \\ \text { PWT } & \text { Paw Withdrawal Threshold } \\ \text { SD } & \text { Sprague-Dawley } \\ \text { SCS } & \text { Spinal Cord Stimulation } \\ \text { SP } & \text { Substance P } \\ \text { SSEP } & \text { Somato Sensory Evoked Potential } \\ \text { TCA } & \text { Tricyclic Antidepressants } \\ \text { TNF } & \text { Tumor Necrosis Factor } \\ \text { TTX } & \text { TetraodonToXine } \\ \text { VRT } & \text { Ventral Root Transection } \\ \text { WDR } & \text { Wide Dynamic Range } \\ & \end{array}$





\section{Chapter}

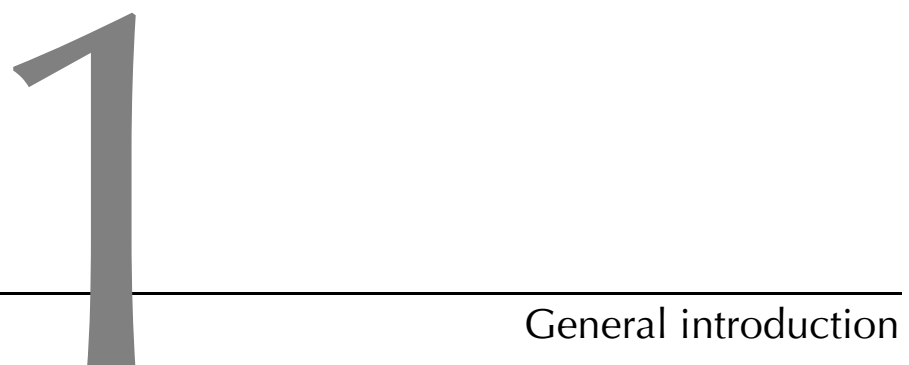





\section{Neuropathic pain}

Neuropathic pain has been defined by the IASP as: "pain initiated or caused by a primary lesion or dysfunction in the nervous system" [132]. Hence, neuropathic pain can be the result of an injury or dysfunction affecting the peripheral or central nervous system as a consequence of direct damage to the nerve, an infection (herpes zoster) or a metabolic disease (diabetes). Neuropathic pain is pathological and constitutes a maladaptive response to a previous injury. This in contrast to nociceptive pain, which functions as a protection mechanism to prevent any further damage. To diagnose neuropathic pain and distinguish it from nociceptive pain it is helpful to analyse the exact quality of somatosensory abnormalities. Patients with neuropathic pain almost always have areas of abnormal sensation or hypersensitivity in the affected area. The abnormal sensations can be presented as spontaneous positive symptoms or paraestesias (i.e. skin crawling sensations or tingling) and spontaneous (not stimulus-induced) ongoing pain (shooting electrical and/or burning sensations). Many patients with neuropathic pain also have evoked pain (i.e. stimulus-induced pain and hypersensitivity). Patients usually report mechanical and thermal hypersensitivity. Two types of hypersensitivity can be distinguished. First, allodynia is defined according to the IASP as pain due to a stimulus which does not normally provoke pain. In cases of mechanical allodynia, even gentle mechanical stimuli such as slight bending of hairs or the touch of clothing can evoke severe pain. Second, hyperalgesia is defined as an increased response to a stimulus which is normally painful, such as a pin prick or exaggerated pain due to heat or cold.

The availability of experimental animal models have increased the knowledge of the molecular and cellular mechanisms underlying neuropathic pain. These animal models, which are almost exclusively based on neuropathic pain induced by a peripheral nerve injury, show comparable signs of hypersensitivity to stimulus-induced pain like mechanical and thermal stimuli as seen in man [95]. However spontaneous pain related behaviors presented in animal models of neuropathic pain are still under debate. The hypersensitivity to induced stimuli are the result from an increase in the sensitivity of primary afferent nociceptors in the vicinity of the injury (peripheral sensitization) $[12,166]$ and from increased excitability of pain transmission neurons located in the spinal cord (central sensitization) [234]. As a consequence, the response of a pain transmission neuron to a stimulus is increased. For instance, one of the major pain transmission neurons in the dorsal horn of the spinal cord, the wide dynamic range (WDR) neurons have shown to exhibit higher spontaneous discharge rates and increased after-discharges to non-noxious stimuli in animals with neuropathic pain $[159,163]$. Increased spinal release of the excitatory neurotransmitter glutamate from the sensitized peripheral afferents is thought to be the initiator of central sensitization. 


\section{Perception and nociception}

Perception begins in the periphery, where the peripheral terminals of primary sensory afferent fibers respond to a variety of stimuli and conveys this information to the dorsal horn of the spinal cord. There are three main types of sensory fibers in the peripheral nervous system, $A \delta$-fibers, $A \beta$-fibers and C-fibers. Each has different properties allowing them to respond to and transmit different types of sensory information. A $\beta$-fibers are large, rapidly conducting myelinated fibers. These fibers have low activation threshold and normally respond to light touch and are responsible for conveying innocuous tactile information and thus do not contribute to pain. By contrast, small diameter, unmyelinated slow conducting Cfibers and medium diameter, thinly myelinated more rapidly conducting A $\delta$-fibers have high activation thresholds and respond to noxious stimuli. Both A $\delta$ - and Cfibers can be termed as nociceptors or 'pain fibers', mediating rapid, acute pain and the delayed diffuse, dull pain respectively. All central terminals of primary afferent fibers terminate in the dorsal horn of the spinal cord, which is organized into different laminae.

These laminae can be distinguished based on their characteristic morphoanatomical qualities as defined by Molander in the rat [146]. Most nociceptive $\mathrm{A} \delta$ - and C-fibers terminate in the superficial laminae I-II (or substantia gelatinosa), whereas A $\beta$-fibers predominately innervate laminae III-V [213]. In the substantia gelatinosa, nociceptive-specific (NS) pain transmission neurons are located and receive input from $A \delta$ - and C-fibers only. Cells which receive input exclusively from $A \beta$-fibers are proprioceptive, only respond to touch and are located in laminae III-VI. A second type of neuron, the WDR which is predominantly found in lamina $V$, receives input from all three types of sensory fibers. Finally, these transmission neurons will project the signals to higher brain centers for the perception of pain or light touch.

The normal signal transduction from the periphery to higher brain centers is coordinated at the level of the spinal cord where the spinal cord acts as a "pain gate". Opening and/or closing of the gate is a balanced process between excitation and inhibition. The main excitatory amino acids (EAA) in the nervous system are glutamate and aspartate and are released from the primary afferents neuron endings. The transmission neurons are under local modulation of GammaAmino Butyric Acid (GABA) releasing interneurons (Figure 1.1). GABA is the main inhibitory neurotransmitter in the central nervous system and GABAergic interneurons can be found in abundance in the superficial layers of the dorsal horn [214]. GABA is thought to exert its effect both through inhibition of presynaptic release of EAAs and post-synaptic by hyperpolarizing post-synaptic neurons. Stimulation of pre-synaptic $\mathrm{GABA}_{\mathrm{B}}$ receptors by GABA suppresses EAA release through the inhibition of $\mathrm{Ca}^{2+}$ influx [139]. Post-synaptically, GABA exerts its effect mainly through the $\mathrm{GABA}_{\mathrm{A}}$ receptor and binding results in an increased $\mathrm{Cl}^{-}$influx. This $\mathrm{Cl}^{-}$influx hyperpolarizes the cell, thereby decreasing the likelihood 
of that neuron propagating an action potential [16] (Figure 1.1). Under physiological conditions the excitation and inhibition are in balance and the spinal pain gate functions correctly. When this balance is disturbed, for example due to damage to peripheral nerves, the spinal pain gate remains open which may result in the development of neuropathic pain [45].

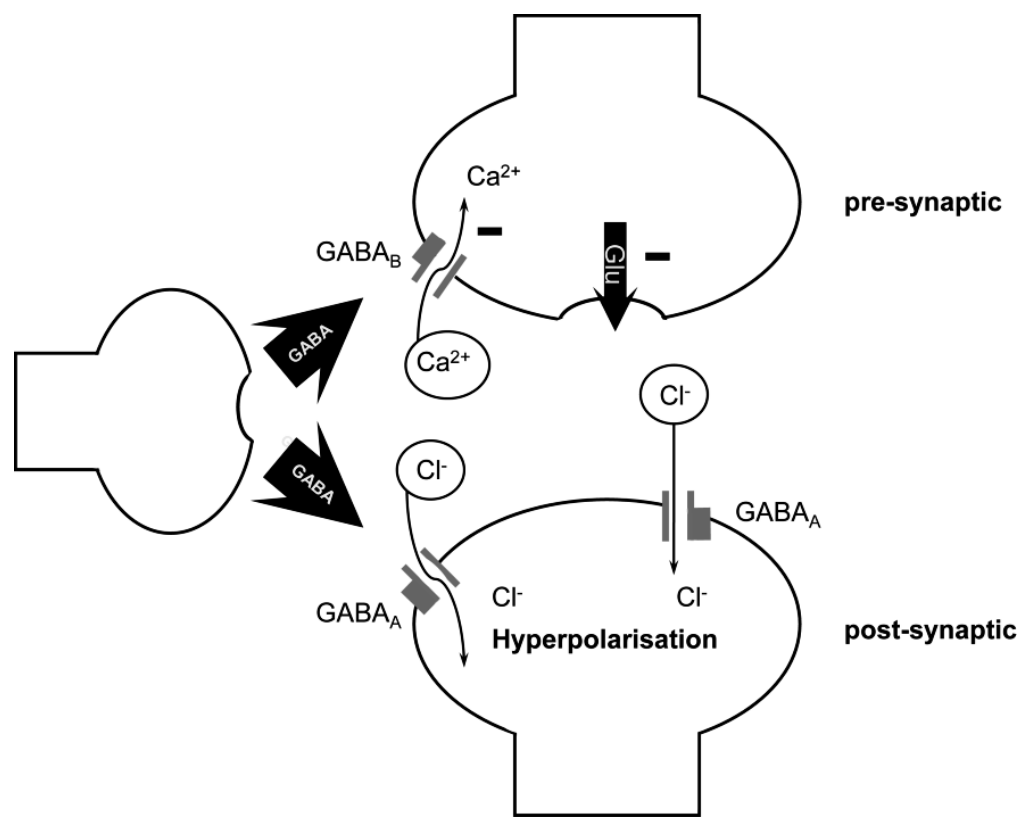

Figure 1.1. Pre- and postsynaptic effect of GABA on signal transduction. Binding of GABA, released from a GABAergic interneuron, to the pre-synaptic $\mathrm{GABA}_{\mathrm{B}}$ receptor results in an influx of $\mathrm{Ca}^{2+}$. This $\mathrm{Ca}^{2+}$ influx results in a decreased glutamate (Glu) release from the pre-synaptic terminals. Binding of GABA to the post-synaptic $\mathrm{GABA}_{\mathrm{A}}$ receptor will result in the influx of $\mathrm{Cl}^{-}$in the post-synaptic cell, thereby hyperpolarizing the post-synaptic neuron. Both the decreased glutamate release from primary afferents and hyperpolarisation of a post-synaptic transmission neuron result in a decreased signal transduction.

\section{Molecular aspects of neuropathic pain}

The increased synaptic efficacy, or central sensitization of post-synaptic neurons in the dorsal horn largely depends on the activation of postsynaptic N-methyl-Daspartic acid (NMDA) receptors [237] and trafficking of the $\alpha$-amino-3-hydroxy-5methyl-4-isoxazolepropionic acid (AMPA) receptor from the cytosol to the postsynaptic membrane. NMDA receptors can be found in abundance in the brain and spinal cord and are crucial for neuronal communication and synaptic function. Different NMDA receptors have been identified based on their sub-unit 
composition. Until now, seven sub-units have been identified: one NR1, four NR2 (A-D) and two NR3 (A and B). Most native NMDA receptors appear to function as heterotetrameric assemblies composed of two NR1 and two NR2 subunits. The NMDA receptors are therefore NR2-containing receptors in which NR1/NR2 heterodimers appear to be the functional unit [25]. In particular, the assembly of NR1 and the NR2B sub-unit are thought to play a crucial role in the occurrence of central sensitization and neuropathic pain $[65,80]$. They can be found in the superficial dorsal horn [140] but are also present on many other spinal neurons [116].
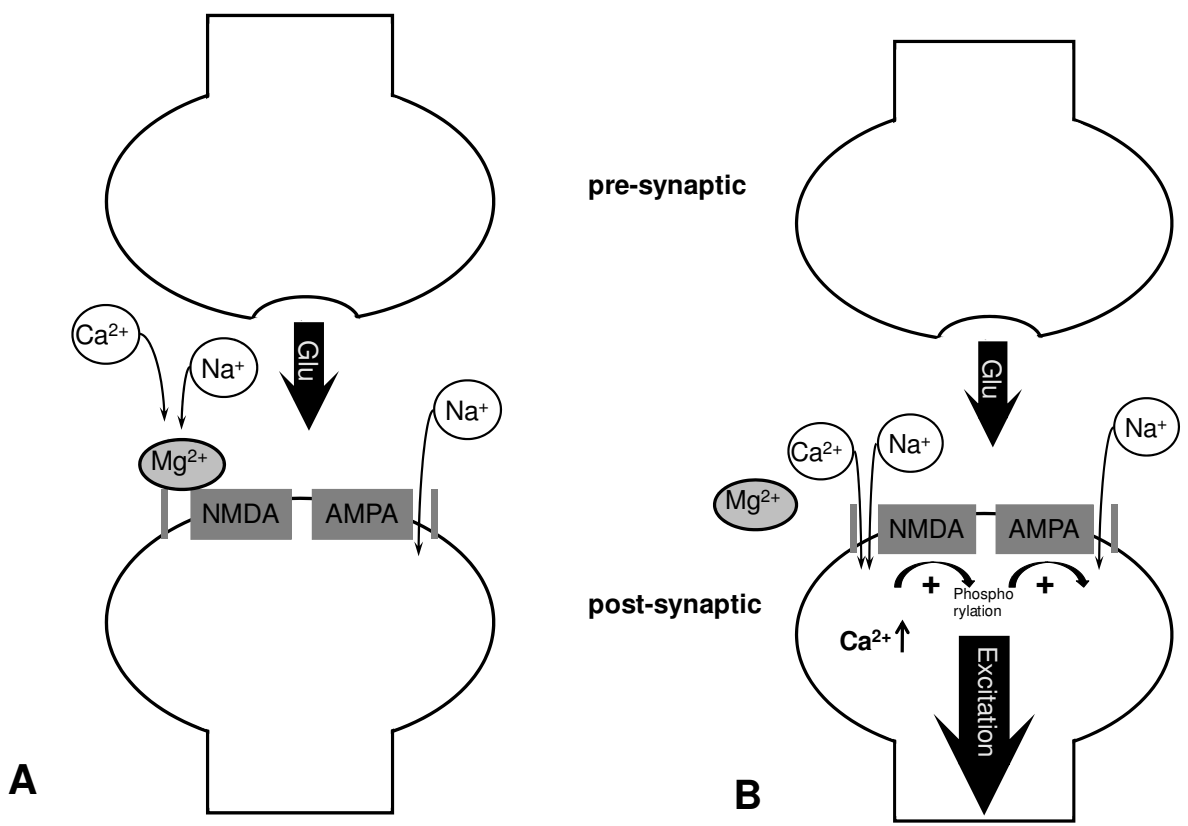

Figure 1.2. (A) $\mathrm{Na}^{+}$influx through the AMPA receptor is not enough to result in excitation. (B) Induction of central sensitization. The central terminals of primary afferents release the neurotransmitter glutamate (Glu). Glutamate binds to ionotropic AMPA and NMDA receptors. Through removal of the $\mathrm{Mg}^{2+}$ block of the NMDA receptor, $\mathrm{Ca}^{2+}$ influx increases, resulting in activation (phosporylation) of the NMDA and AMPA receptors by proteinkinases. Activated NMDA receptors become permeable to $\mathrm{Na}^{+}$, thereby contributing to the signal transduction. The increased $\mathrm{Ca}^{2+}$ furthermore results in the trafficking of AMPA and NMDA receptors to the cell membrane.

Normal glutamatergic synaptic transmission occurs through non-NMDA receptors such as the AMPA receptor. NMDA receptor contribution is limited due to the voltage-dependent $\mathrm{Mg}^{2+}$ block acting on the NMDA receptor [128]. As a result of the increased afferent input the $\mathrm{Mg}^{2+}$ block is removed, initiating the activation of 
protein kinases through the influx of $\mathrm{Ca}^{2+}$, altering channel opening time of the NMDA and AMPA receptor through receptor phosphorylation and promotion of trafficking of AMPA and NMDA receptors to the synaptic membrane $[82,236]$. This results in a hypersensitive state of that neuron to input from primary afferents and neuropathic pain (Figure 1.2).

Alongside the activation of the NMDA receptor, the inhibitory system is impaired and animals with a nerve injury show a reduction in the level of GABA [78] and its synthesizing enzyme glutamate decarboxylase [147] in the dorsal horn. Therefore, the hypersensitivity in animal models with a peripheral nerve injury is attributed to the increased activation of the NMDA receptor and the impaired GABAergic inhibition [45].

Taken together, increased hypersensitivity through activation of NMDA receptors, trafficking of AMPA receptors and impaired GABAergic inhibition in the dorsal horn of the spinal cord results in an permanent opening of the spinal pain gait resulting in neuropathic pain.

\section{Complex Regional Pain Syndrome}

Already in the $19^{\text {th }}$ century, Claude Bernard described symptoms of neuropathic pain associated with intense autonomic dysregulation in soldiers from the American Civil War, which later became to know as "causalgia" or Complex Regional Pain Syndrome (CRPS) type 2 (CRPS-2). In patients with CRPS, neuropathic pain predominates and is frequently associated with positive sensory symptoms, including allodynia and hyperalgesia [235] and is furthermore characterized by autonomic and motor disturbances [133]. The incidence of CRPS ranges from 5.5 to 26.2 per 100.000 person years [39,177] and predominantly affects women. The current IASP diagnostic criteria make a distinction between CRPS type 1 (CRPS-1) (formally known as Reflex Sympathetic Dystrophy), where there is no clear cause of the syndrome and CRPS-2 where there is clear evidence of nerve injury [133]. The underlying mechanisms of CRPS are largely unknown, although hyperactivity of the autonomic nervous system is often suggested to be associated with CRPS. Furthermore, other mechanisms besides increased activity of the sympathetic nervous system may also account for signs of sweating, trophic changes and vasoconstriction [51,63,212] as sympatholytic blocks are not always successful [23]. The neuropathic pain in patients with CRPS can be the result of peripheral and central sensitization, as damaged sensory neurons may barrage the dorsal horn with input, resulting in spontaneous pain, allodynia and hyperalgesia. On the other hand, in patients with CRPS-1, where there are no clear signs of nerve damage, local and/or central inflammatory processes are thought to play a pivotal role [198]. Fluid from blisters on the affected extremities of CRPS-1 patients reveals pro-inflammatory mediators like interleukin- 6 and tumor necrosis factor-alpha (TNF- $\alpha$ ) [77]. Also the presence 
of pro-inflammatory cytokines interleukin-1 and TNF- $\alpha$ in the central nervous system are reported in CRPS-1 patients [1,219].

Numerous experimental animal models of CRPS have been developed in rats based on clinical observations. These models entail direct damage to the peripheral nerve (sciatic nerve or its branches) $[10,41,54,187,230]$, the spinal nerve [92,189] or Dorsal Root Ganglion (DRG) [200] and mimic the clinical signs like pain, vasomotor changes, oedema and motor/trophic changes to some extent. Because of the induced direct damage to the nerves, these animal models can be best described as examples of CRPS type 2. Also attempts have been undertaken to model CRPS type 1. One of the first models, the L5 Ventral Root Transection (L5 VRT) model was introduced in the rat in 2002 [107]. In order to evaluate the role of inflammation in the absence of any direct damage to sensory neurons, Li and colleagues developed an animal rat model in which only the L5 ventral motor axons were transected [107]. Hence, sensory afferents located on the dorsal root remained intact. They demonstrated that signs of neuropathic pain (mechanical allodynia and thermal hyperalgesia) could develop without direct damage to primary sensory neurons as long as their peripheral axons are exposed to an environment of Wallerian degeneration. Two other examples of animal models for CRPS-1 are the tibial fracture model [64] and the chronic post-ischemia pain model [28]. Both models exhibit signs of mechanical allodynia, thermal hyperalgesia and oedema.

The term syndrome is derived from the Greek word sundromè and means "run together" and as many syndromes CRPS is seen as a multifactorial disease. In such a disease, environmental and genetic factors both play a role during the development of CRPS. The first link between CRPS and a possible genetic factor was provided by Mailis and Wade [122] and since then the role of genes that might predispose to the development of CRPS have been appreciated $[40,62,89]$. These genes are associated with the human leukocyte antigen (HLA) system, which is part of the major histocompatability complex (MHC) and are thought to mainly predispose for the occurrence of dystonia in CRPS-1 patients. Although no association was reported between the HLA system and neuropathic pain in these patients, certain allelic variants of the MHC could influence susceptibility to the development of neuropathic pain in rats [46]. To study the contribution of genetic factors in a disease one can use family or twin studies. At the experimental level most genetic studies use mice. The laboratory mouse is exceptionally suitable for this purpose because it displays easy mutagenesis and over $95 \%$ of the mouse genome is similar to that in human $[37,158]$. Furthermore, a wide range of inbred strains of mice are available which allows genetic control within a strain. Genetic variability between strains can also be compared. Different mouse strains have been reported to respond different to the induction of pain, thereby indicating a variability between strains [144]. For instance, after a peripheral nerve injury, the 129 mouse strain was more susceptible to the manifestation of mechanical hypersensitivity as compared to the C57BL/6 mouse strain [144]. By determining 
genetic predispositions to neuropathic pain, pharmacogenomics holds the promise of an individually tailored approach, not only to the effective treatment of neuropathic pain, but also in the prevention of unwanted reactions and adverse effects.

\section{Treatment of neuropathic pain}

Because neuropathic pain is reported to be the most debilitating symptom of CRPS, treatment of the pain is the first priority. Although different treatment strategies are proposed for the treatment of neuropathic pain in patients with CRPS [204,205], the evidence for effective pain relief remains limited. The most commonly used drugs in neuropathic pain as in CRPS are the tricyclic antidepressants (TCAs). The TCA amitriptyline has the best documented efficacy in the treatment of neuropathic pain [18], although there is no evidence that TCAs are effective in reducing pain in patients with CRPS-1 [160].The antiepileptic drug gabapentin (Neurontin) is effective in the treatment of painful diabetic neuropathy and postherpetic neuralgia [4], probably by inhibiting the glutamate release [27]. However, its efficacy in CRPS-1 and CRPS-2 has not been proven [5,221]. Other drugs, like the 2-adrenergic agonist clonidine and the $\mathrm{GABA}_{\mathrm{B}}$ receptor agonist baclofen have shown to contribute to the pain relieve in patients with neuropathic pain $[73,168]$. Another drug which is reported to be effective in neuropathic pain is ketamine. Ketamine, a non-competitive NMDA-receptor antagonist, is used in a sub-anaesthetic dose and is appreciated in a wide range of pain settings [224] since the 1960s. Although appreciated, the clinical evidence in successful pain relief is moderate to weak [30,74,192]. In animal models of neuropathic pain, ketamine was able to reduce pain related behavior and decreased dorsal horn neuronal hyperactivity $[126,152,165]$. Ketamine exerts its effect through the binding to the phenylcycline (PCP) site inside the NMDA receptor thereby blocking the $\mathrm{Ca}^{2+}$ influx [170]. Although it has been suggested that ketamine may mediate its analgesic effect through other receptors like the opiod receptor in the central nervous system (CNS) [31], the high affinity of ketamine for the NMDA makes it likely that the analgesic effect of ketamine acts primarily through the NMDA receptor $[49,130]$.

Because of the widespread distribution of the NMDA receptor in the CNS, the use of ketamine as an analgesic is hampered by a variety of clinical side effects. These include hallucinations, dizziness, vivid dreams and confusion [38], and thus ketamine is not used as a standard therapy to treat neuropathic pain. Even though there is solid evidence for pharmacotherapeutic treatment, there are still a large group of patients that are pharmaco-resistant and thereby do not receive adequate treatment. These patients might benefit from neuromodulation of the central nervous system, for instance spinal cord stimulation. 


\section{Spinal Cord Stimulation}

The electrical modulation of the dorsal column or Spinal Cord Stimulation (SCS) for neuropathic pain was first applied in 1967 by Shealy [188]. The pain relieving effect of SCS was then explained by the so called Gate Control Theory by Melzack an Wall [131]. This theory stated that the dorsal horn of the spinal cord acts as a gate, which can be opened and closed. According to the theory, the transmission cells ( $T$ cells (NS and WDR)), which are activated by both A $\beta$ fibers and $\mathrm{C} / \mathrm{A} \delta$-fibers, activate neural mechanisms that produce pain perception. $\mathrm{A}$ Gate cell, or G cell (possibly a GABAergic interneuron) is capable of modulating the activity in afferent pathways (of both large and small fibers) to the T cell, before it gets to the T cell. The G cell activity is, in turn, modulated by activity in both large and small primary afferent fibers. Activation of the small A $\delta$ or C-fibers inhibits the activation of the $G$ cell thereby keeping the spinal pain gate open. Activation of A $\beta$-fibers (for instance by SCS) however, activate the G-cell, which in turn inhibits activation of the T cell by $A \beta, C$ and $A \delta$-fibers, thereby closing the gate (Figure 1.3).

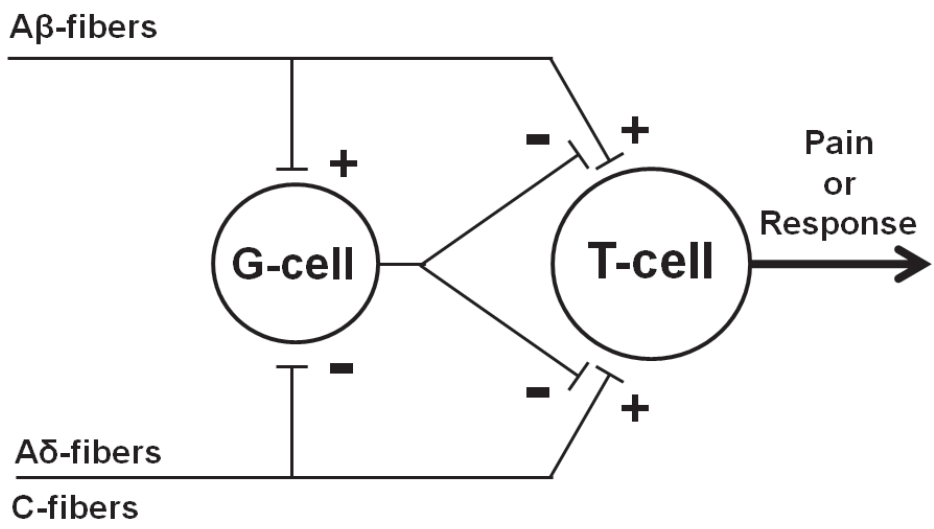

Figure 1.3. The Gate Control Theory is based on the interaction of different classes of neurons in the dorsal horn: the A $\delta$ - and C-fibers that hold the gate open through inhibition of the G-cell and the A $\beta$ fibers that tend to close the gate by activating the G-cell. The activated G-cell inhibits signal transmission at the T-cell site by the decreased release of EAA from the primary afferents.

Since then, SCS has emerged as a therapy for especially pharmacological resistant neuropathic pain. In particular in patients with Failed Back Surgery Syndrome (FBSS) and CRPS-1, SCS has proven to offer satisfactory and long lasting pain relief in about $60 \%$ of the patients. Consequently, still $40 \%$ of these patients with FBSS or CRPS-1 do not respond to SCS with satisfactory pain relief (non-responders tot SCS) $[85,86,98]$. 
In 1995, Meyerson and colleagues developed and experimental animal model of neuropathic pain and SCS in the rat [135]. With this animal model, which used SCS parameters comparable to that used in patients a comparable percentage of responders to SCS was noted as observed in patients with CRPS and FBSS $[85,101]$. This animal model made it possible to study the effect of SCS on neuropathic pain at the cellular and biochemical level. For instance, it was shown that SCS resulted in a decreased tactile hypersensitivity (responders to SCS) and this correlated with increased extracellular GABA levels in the dorsal horn in rats with neuropathic pain [206]. Subsequent experiments revealed that SCS induced a decrease in the extracellular EAAs glutamate and aspartate in the dorsal horn and an increase in GABA levels in animals with neuropathic pain [36]. Interestingly, in animals with neuropathic pain that did not responded to SCS (non-responders), both levels of EAAs and GABA were almost unaffected by SCS $[36,206]$. The SCS induced EAA decrease in SCS responders could be prevented through the intrathecal application of a selective GABA $\mathrm{B}_{\mathrm{B}}$ antagonist (CGP 55845) [36]. Furthermore, the intrathecal application of baclofen or gabapentin could potentiate the attenuation of neuropathic pain by SCS [35,231] Electrophysiological evidence showed that in rats with neuropathic pain, SCS induced long lasting depression of hyper-excitable WDRs by attenuating both spontaneous and evoked after-discharges [242] and that SCS at the same time reduced the Cfiber evoked central sensitization [232]. These results indicate a spinal segmental modulation of excitatory and inhibitory molecules to be pivotal in the mechanisms underlying the pain relieving effects of SCS. From a clinical point of view this was further substantiated by a combination of SCS and local intrathecal administration of baclofen in patients with neuropathic pain [109,179].

Although the primary effect of SCS has been attributed to the antidromic activation of ascending dorsal column fibers inducing segmental neurochemical changes in the dorsal horn, there is evidence that orthodromic activation occurs involving supraspinal mechanisms via a loop in which the descending dorsolateral funiculus (DLF) plays a role in the attenuation of neuropathic pain $[53,173]$.

Despite the success of spinal cord stimulation in patients with FBSS or CRPS-1, approximately $40 \%$ of patients with a permanent implanted SCS device report unsatisfactory pain relief $[85,86]$ and patients with good pain relief report that the beneficial effect of SCS dissolves over time $[87,88]$. Furthermore, 30\% of patients do not respond to the mandatory trial SCS and thereby do not receive a permanent implant [85,99]. Taken together, there is still the need for improvement on the SCS therapy in neuropathic pain syndromes.

\section{Improvement of SCS in the treatment of neuropathic pain}

An important issue in further improvement of SCS as treatment for neuropathic pain is the establishment of predictors or prognostic factors for the success of SCS. 
It is important to select the patients with care and use standardized selection criteria for misdiagnosis because the latter may lead to inappropriate treatment $[68,69]$. Using specific prognostic factors can help to increase the success of pain relief. In patients with CRPS-1, it was shown that an absent or altered conductivity in the dorsal column, measured by somatosensory evoked potentials (SSEPs), was a negative predictor for success of SCS induced pain relief [193]. Furthermore, the absence of brush-evoked allodynia was associated with a good outcome of SCS [197]. Alongside better patient selection, optimal timing of the procedure might improve the outcome of the treatment [203]. From a mechanistically point of view, early intervention in the process of central sensitization might result in prevention, or at least a delay in the onset of central sensitization, thereby possibly preventing or delaying the induction of neuropathic pain. With respect to the success of spinal cord stimulation several case studies have reported an improved outcome when SCS was applied at an early stage of CRPS- 1 $[71,178,223]$. Therefore, the combination of stringent patient selection and optimal timing of the intervention might improve overall outcome of the pain relieving effect of SCS.

Another strategy may be the combined treatment of neuropathic pain using both SCS neuromodulation and pharmacotherapy. From animal studies it became clear that the combined use of sub-effective dosages of currently used drugs combined with SCS could potentiate the effect of SCS induced pain relief. In animals with neuropathic pain that did not respond to SCS (non-responders), the intrathecal addition of a low dose of muscimol $\left(\mathrm{GABA}_{\mathrm{A}}\right.$ agonist) and baclofen $\left(\mathrm{GABA}_{B}\right.$ agonist) resulted in successful pain relief in these animals [33]. The combined treatment of baclofen and SCS was successfully applied in a pilot study in patients with neuropathic pain [109]. Also the combined use of SCS and low dose of gabapentin and pregabalin (GABA analogues) were able to convert SCS nonresponder into SCS responding rats [231]. Furthermore, clonidine and serotonin could also convert SCS non-responder into SCS responders presumably by facilitating GABAergic inhibition [181,201]. Recently it was reported that intrathecal clonidine in combination with SCS could enhance the pain relieving effect of SCS in patients with neuropathic pain. All pharmacological augmentation of SCS previously described is directed at the inhibitory component of spinal transmission (spinal pain gate). It might therefore be of interest to approach the problem of non-responders to SCS not only from the inhibitory side, but also from the excitatory side i.e. antagonising the NMDA receptor in combination with SCS. Taken together, the combined use of pharmacotherapy and spinal cord stimulation looks promising, especially when the effect of SCS appears to diminish over time. 


\section{Measurement of pain related behavior in animal models of neuropathic pain}

Assessing pain related behaviour in animals is mostly based on evoked withdrawal responses to active stimuli (von Frey monofilaments or heat source) $[67,141]$. However, these evoked responses measure not pain itself but rather the hypersensitivity that often accompanies pain. Indeed, patients with neuropathic pain report mechanical allodynia and/or thermal hyperalgesia, however the spontaneous pain is reported more frequently [6].

To test the effect of a painful procedure in animals, hypersensitivity to mechanical stimuli is traditionally tested using von Frey monofilaments. Each filament varies in thickness and thereby exerts a different amount of pressure. The smallest filament that elicits a brisk withdrawal response is considered the threshold stimulus. When an animal responds to a "smaller" filament (i.e. less pressure) after the painful intervention, as compared to before the intervention, this is interpreted as pain. This pressure or mechanical induced pain is referred to as mechanical allodynia. The same principle is true for thermal hyperalgesia, where decreased latencies to a thermal source after a painful intervention is interpreted as pain. Although these tools are of use in the assessment of behavioral changes, pain is a more complex process, including the processing and modulation in higher brain centers. The withdrawal from mechanical or heat stimuli are predominantly a

spinally mediated reflex and do not necessarily include activation of higher brain centers. It was therefore proposed that the measurement of spontaneous behavior emitted by the animal might be a better representation of the neuropathic pain that animal is experiencing [142]. In this respect, the CatWalk, which is an automated technique to measure the spontaneous gait of the animal, could proof useful. The CatWalk has been suggested to be an alternative tool to measure mechanical allodynia in animals with inflammatory or neuropathic pain $[58,226]$. Gait analysis represent to a certain extend spontaneous behavior and thus changes in gait, due to neuropathic pain, might be a representation of spontaneous pain and could therefore be related to pain as presented in the clinic. The CatWalk consists of a glass walkway, which the animal has to cross voluntarily and the locomotion can be analyzed using individual paw parameters and parameters related to interlimb coordination.

\section{Objective and research questions}

The objectives of this thesis were to 1 . Develop a mouse model of neuropathic pain and spinal cord stimulation and 2. Increase the number of responders to spinal cord stimulation in an experimental model of neuropathic pain.

In the context of the first objective, a mouse model for CRPS-1 was developed and next spinal cord stimulation was implemented in a mouse model of neuropathic pain. The CatWalk was used in addition to the von Frey monofilaments to assess 
pain related behaviour and the effect of spinal cord stimulation on pain related behaviour.

With respect to the second objective the possibility to increase the number of responders to spinal cord stimulation in a rat model of neuropathic pain, either through an early intervention (early SCS) in the process of central sensitization or through the use of drug-enhanced spinal cord stimulation, was explored.

The following research questions according to the two objectives were formulated:

1) a. Does indirect inflammation of uninjured sensory afferents in the dorsal root result in signs of neuropathic pain in mice and are these changes strain dependent?

b. Does spinal cord stimulation in mice with neuropathic pain result in the attenuation of neuropathic pain?

2) a. Does early spinal cord stimulation result in an increased number of responders to spinal cord stimulation as compared to late spinal cord stimulation in a rat model of neuropathic pain?

b. Does the combined use of a sub-therapeutic intrathecal dose of ketamine and spinal cord stimulation result in an increased number of responders to spinal cord stimulation as compared to spinal cord stimulation per se in a rat model of neuropathic pain?

In chapter 2 the $\mathrm{C} 57 \mathrm{BL} / 6$ and 129J mice were used to test if an indirect inflammation of the dorsal root by means of a L5 ventral root transection (VRT) results in behavioral signs of neuropathic pain using the von Frey monofilaments and the CatWalk. This was based on the results of a L5 VRT as previously reported in the rat [107]. Because the L5 VRT did not result in any changes in behaviour the partial ligation of the sciatic nerve (Seltzer) model was used in the following experiments for the induction of neuropathic pain. The Seltzer model is known to induce signs of neuropathic pain in mice [125] and in rats [187]. This model of neuropathic pain is frequently used in combination with spinal cord stimulation $[36,181,197]$. Chapter 3 and 4 are devoted to the question whether spinal cord stimulation in $\mathrm{C} 57 \mathrm{BL} / 6$ mice with behavioral signs of neuropathic pain is technically possible and if this results in the alleviation of the neuropathic pain. The behavioral changes were assessed using the von Frey monofilaments. The CatWalk gait analysis was included for the evaluation for the use in the assessment of behavioural signs of neuropathic pain and the effect of spinal cord stimulation on this pain related behaviour. Chapter 5 deals with the question if early spinal cord stimulation (after 1 day of neuropathic pain) results in more "responders" to spinal cord stimulation by comparing the results with that of late spinal cord stimulation (after 16 days of neuropathic pain). Behavioral changes were assessed based on paw withdrawal thresholds using the von Frey monofilaments. The effect of a low sub-effective dose of intrathecal ketamine in 
combination with spinal cord stimulation in animals with neuropathic pain that did not respond to spinal cord stimulation as a monotherapy is studied in chapter 6. Chapter 7 summarizes the main research findings of this thesis and offers a general discussion, addressing some considerations related to the research and several recommendations for clinical practice and further research. 



\section{Part I}

Mouse models 



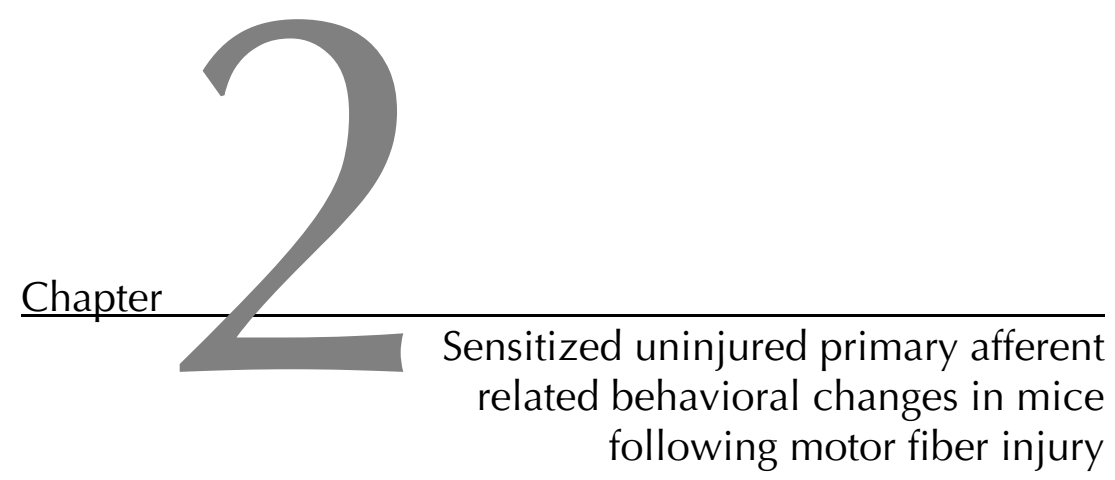

M. Truin

G. Koopmans

E. Pragt

M. van Kleef

E.A.J. Joosten 


\section{Abstract}

\section{Background}

In patients with chronic neuropathic pain there is often no clear sign of damage to peripheral nerves. It is believed that ectopic discharges from uninjured sensory afferents and not the injured ones are important for the development of neuropathic pain. Biochemical changes observed after selective lesioning of motor fibers (L5 Ventral Root Transection (VRT)) suggest an important role for inflammatory mediators in the development of neuropathic pain in mice. However, it is not clear if injury to the L5 motor fibers results in similar behavioral symptoms of neuropathic pain in mice as reported in rats. We therefore studied the behavioral changes after selective lesioning of L5 motor fibers in C57BL/5 mice as well as in the more sensitive 129J mice.

\section{Materials and Methods}

The behavioral effect of the L5 VRT (C57BL/6 $(N=11)$ and 129J $(N=11))$ and sham-VRT $(\mathrm{C} 57 \mathrm{BL} / 6(N=5)$ and $129 \mathrm{~J}(N=7))$ was tested using von Frey monofilaments and the CatWalk gait analysis. The chronic constriction injury model $(\mathrm{CCl})$ in $\mathrm{C} 57 \mathrm{BL} / 6$ mice was used as a control group.

\section{Results}

After selective sectioning of L5 motor fibers, no behavioral signs of neuropathic pain could be observed in C57BL/6 and 129J mice over time using von Frey monofilaments and the CatWalk gait analysis.

\section{Conclusions}

Selective lesioning of motor fibers in two mouse strains did not result in behavioral signs of neuropathic pain. 


\section{Introduction}

Chronic neuropathic pain can be a consequence of mechanical and/or inflammatory responses after peripheral nerve injury [105]. Whether peripheral nerve injury is a prerequisite for chronic neuropathic pain is, however, a matter of debate. For instance, the Complex Regional Pain syndrome type 1 (CRPS-1) typically develops with small or no obvious nerve lesion at the periphery, although spontaneous pain, hyperalgesia and allodynia are present. Important characteristics of CRPS- 1 are signs of inflammation in the periphery $[77,182]$. Almost all animal models of chronic neuropathic pain induce direct $[10,41,92,187]$ damage to peripheral sensory neurons and it has been suggested that ectopic discharges from injured Dorsal Root Ganglia (DRG) neurons might contribute to neuropathic pain $[189,244]$. However, it has also been suggested that ectopic discharges from uninjured afferents contribute to the development of neuropathic pain [136,238]. Indeed, in the L5 spinal nerve lesion model for chronic neuropathic pain, allodynia and hyperalgesia could not be reversed or prevented by transection of the L5 dorsal root [108], indicating that the uninjured afferents and not the injured ones are important for the development of neuropathic pain. In these models, including the chronic constriction injury $(\mathrm{CCl})$ model, signs of neuro-inflammation and Wallerian degeneration have been demonstrated to contribute to the generation of neuropathic pain $[32,149,167]$.

In order to evaluate the role of inflammation in the absence of any direct damage to sensory neurons, Li and colleges developed a rat model in which only the L5 ventral root (motor axons) is transected [107]. They demonstrated that in the rat neuropathic pain could develop without direct damage to primary sensory neurons as long as their peripheral axons where exposed to an environment of Wallerian degeneration. Furthermore, recent data suggest an important contribution of increased TNF- $\alpha$ in the up-regulation of the tetraodontoxinesensitive (TTX-S) sodium channels Nav 1.3 and Nav 1.8 in dorsal root ganglia (DRG) following selective transection of L5 motor fibers in mice [72], although no behavioral data on these mice are reported. The over-expression of Nav 1.3 and Nav 1.8 is thought to be critical for the development of neuropathic pain $[11,44,91]$.

Most rat models of neuropathic pain have been implemented in the mouse $[125,144,149,191]$ and then became a valuable addition to study the underlying mechanisms of the development and maintenance of neuropathic pain. Furthermore, it was also reported that distinct inbred mouse strains respond differently to peripheral nerve injury, thereby presenting the possibility to study the genetic basis of pain. For instance, the 129/J mouse strain was shown to be highly sensitive to a peripheral nerve injury compared to the $\mathrm{C} 57 \mathrm{BL} / 6$ mouse strain [144].

In the present study, we aimed at understanding the behavioral consequences of an indirect inflammation of primary sensory neurons using selective lesioning of 
the $\mathrm{L} 5$ motor fibers in the $\mathrm{C} 57 \mathrm{BL} / 6$ and $129 \mathrm{~J}$ mouse. In order to be able to detect sensory related behavioral changes we used the von Frey monofilaments in combination with the CatWalk gait analysis. The CatWalk is suggested to be a very sensitive tool to detect even small sensory and/or motor related gait changes in various experimental pain models $[58,226]$.

\section{Materials and methods}

\section{Animals and surgery}

In this experiment, a total of $27 \mathrm{C} 57 \mathrm{BL} / 6$ and 17 129 J mice, weighing 20-25 g, were used. The animals were individually housed under temperature controlled conditions at $21 \pm 1^{\circ} \mathrm{C}$, with a normal 12:12 h light/dark cycle. The animals had free access to food pellets and water. All experiments were performed in accordance with the European Directive for the Protection of Vertebrate Animals used for Experimental and Other Scientific Purposes (86/609/EU) and were approved by the Committee for Experiments on Animals of Maastricht, The Netherlands. Surgery was performed in anesthetized animals under aseptic conditions. Induction and maintenance of general anesthesia was performed by isoflurane inhalation. Constant body temperature was maintained by placing the animal on an automated heating pad (37.5 $\left.\pm 0.5^{\circ} \mathrm{C}\right)$, (IDEE, University Maastricht). If signs of extreme weight loss ( $>15 \%$ of bodyweight at delivery), absence of grooming or cachectic behavior were observed, the animal was euthanized. Two different injury procedures were used to induce tactile hypersensitivity. The L5 Ventral Root Transection (VRT) was performed according to the procedures described by Li et al. [107]. Briefly, after a midline skin incision in the L4-S1 region, the left L5 vertebra was freed of its muscular attachment. An L5 hemilaminectomy was performed, and the dura mater and arachnoid membrane were incised. The L5 ventral root was identified as it lays at the most lateral side of the spinal canal, just beneath the dorsal root. The ventral root was gently pulled out and carefully transected at two places approximately 1-2 mm apart and this portion was removed. In the sham group, all procedures of operation were identical to the experimental group, except that the exposed ventral root was not transected. After surgery, the wound was washed with saline and closed in layers with 5-0 silk sutures. The $\mathrm{CCl}$ model was used as a control group. The chromic gut used in this model induces local inflammation, invasion of immune cells and production of immune modulators [26,94,148,157], which contributes to the development and signs of neuropathic pain. The procedure was performed as described by Bennett and Xie [10] with minor modifications [149]. Shortly, the right sciatic nerve was exposed and 4 ligatures of 8.0 non-absorbable silk were loosely placed around the nerve, proximal to the sciatic trifurcation, with a distance between the ligatures of approximately $1 \mathrm{~mm}$. In the sham group, all procedures of operation were identical to the experimental group, with the 
exception of the placement of the ligatures around the sciatic nerve. After surgery, the wound was washed with saline and closed in layers with 5-0 silk sutures.

\section{Behavioral testing}

Paw withdrawal thresholds (PWTs) were tested with the use of von Frey monofilaments. All animals were tested before the nerve injury (pre-nerve injury), 7, 14 and 28 days post-operative (DPO). Testing took place in a set of Plexiglas cages with an elevated wire mesh floor. Prior to the testing, the animals were allowed to adapt for 60 minutes. After acclimatization, the von Frey filaments were applied, in an order of increasing stiffness, through the wire mesh floor to the mid-plantar surface of the left or right hind paw until the filaments bent slightly. Testing was performed following the "up and down" method [24]. The animals were tested using 10 von Frey monofilaments (Stoelting CO, IL, USA) with logarithmically incremental stiffness $(0.008,0.04,0.07,0.16,0.4,0.6,1.0$, $1.4,2.0$ and $4 \mathrm{~g})$, corresponding to the linear perceived intensity $(1.65,2.36$, $2.44,2.83,3.22,3.61,3.84,4.08,4.17,4.31$ and 4.56). These values are achieved according to the formula: $\log 10$ ( $\mathrm{mg} \times 10$ ) (Stoelting). Additionally, the CatWalk gait analysis system was used to test possible gait changes resulting from the VRT or CCl operation. The CatWalk analysis has been described in detail for rat and mouse $[66,96,225]$. In short, the CatWalk system consists of a glass walkway which contains light from a white fluorescent source. The light rays from this source are completely reflected internally. When an object touches the glass runway, the light is reflected downwards, where it is detected by a video camera (Sony 3CCD Color Video Camera; DXC-990/990P). This signal is then digitized, which allows analysis by the CatWalk program software [66]. With the CatWalk, a vast variety of static and dynamic gait parameters can be measured. Some of these parameters, including mean intensity of paw placement and stance duration of the hind paw, have been linked to mechanical allodynia and neuropathic pain [226]. In the present study the following individual paw parameters were used: 1 . Regularity index (degree of interlimb coordination), 2. Mean Intensity (average intensity of the pixels at the maximum paw-floor contact, a measure of the relative force being exerted on the floor i.e. favoring), 3. Stance Phase Duration (time contact of the paw with the glass floor, another measure of favoring) and 4. Print Area (total surface area of the glass floor contacted by the paw during the stance phase).

\section{Statistical analysis}

The tactile withdrawal thresholds to von Frey filaments are presented as mean \pm standard error of the mean (SEM) for the perceived intensity followed by the withdrawal thresholds in grams. The CatWalk parameter Intensity is expressed in arbitrary units (au), Print Area in $\mathrm{mm} 2$ and Stance duration in seconds. For 
statistical analysis of differences in the withdrawal thresholds over time, the nonparametric Friedman test, followed by Dunn's post hoc test, was used. For comparison between VRT and Sham-VRT, data were analyzed using a twofactorial ANOVA with the factors time and intervention, with time as repeated measures factor. For comparison between strains data were analyzed using a twofactorial ANOVA with the factors time and strain, with time as repeated measures factor the different groups. One-way ANOVA and unpaired students t-test were used when appropriate. $p<0.05$ was considered to be statistically significant in all tests.

\section{Results}

\section{General observations}

All the operated mice appeared healthy and well groomed. Following an initial drop in the first days after injury, bodyweight steadily increased in the three groups and no differences between the groups were noted during the 4 weeks follow-up period (data not shown).

\section{Development of mechanical hypersensitivity (von Frey)}

Pre-nerve injury PWTs of all the groups were comparable (Figure 2.1A-D). In the $\mathrm{C} 57 \mathrm{BL} / 6$ mice that received the $\mathrm{CCI}(\mathrm{N}=11)$, PWT of the ipsilateral paw $2.3 \pm$ $0.1(0.6 \pm 0.1 \mathrm{~g})$ was significantly decreased at DPO 28 as compared to pre-nerve injury PWT $3.8 \pm 0.1(1.0 \pm 0.1 \mathrm{~g})(p<0.01)$ (Figure 2.1A). PWTs of the ipsilateral paw of the $\mathrm{C} 57 \mathrm{BL} / 6$ mice after L5 VRT $(N=11)$ did not differ between pre-nerve injury values $4.0 \pm 0.1(0.8 \pm 0.1 \mathrm{~g})$ and PWTs at DPO $73.8 \pm 0.1(0.6 \pm 0.1 \mathrm{~g})(p$ $>0.05)$. At DPO 28, PWT of the ipsilateral paw was $3.8 \pm 0.1(0.8 \pm 0.2 \mathrm{~g})$, which was not statistically different compared to pre-nerve injury PWTs (Figure 2.1A). As for the 129J animals that received the L5 VRT $(N=10)$, pre-nerve injury PWT of the ipsilateral paw was $4.1 \pm 0.1(1.1 \pm 0.1 \mathrm{~g})$. Seven days after the L5 VRT, PWT of the ipsilateral paw was $4.0 \pm 0.1(0.6 \pm 0.1 \mathrm{~g})$ which did not significantly differ from pre-nerve injury PWT (Figure 2.1B). No effect on withdrawal thresholds of the ipsilateral paw could be noted after the sham L5 VRT in both strains (Figure 2.1 A and $B)$. 

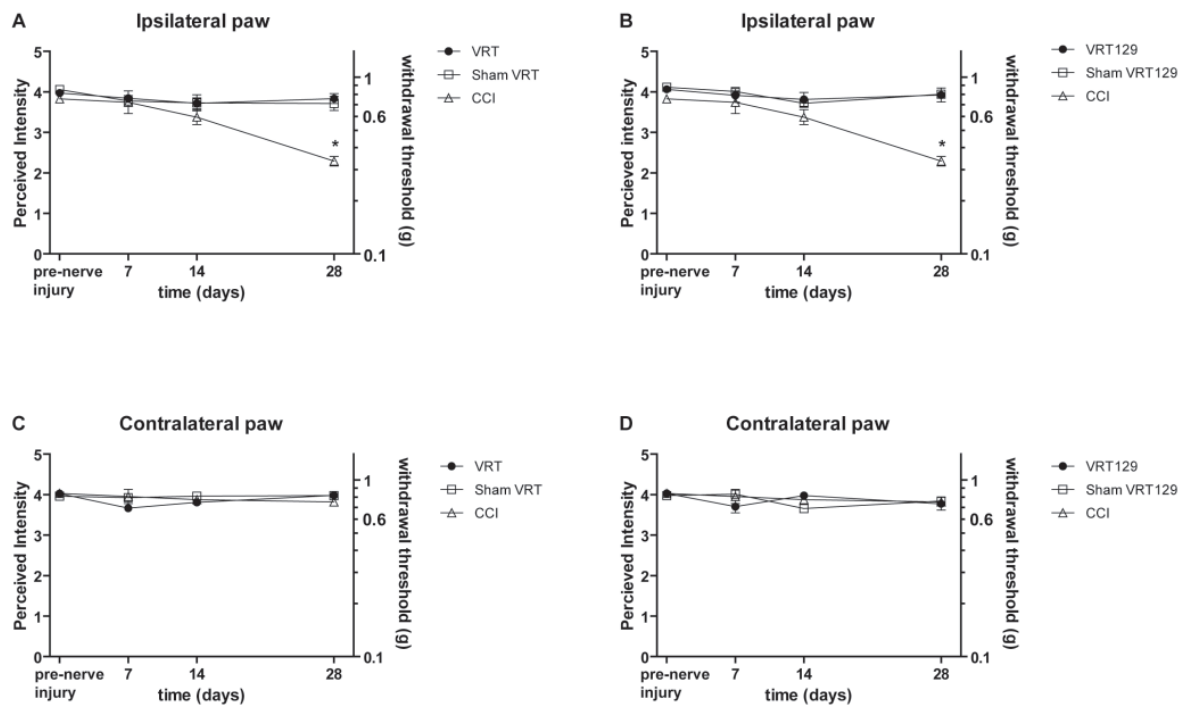

Figure 2.1. Withdrawal thresholds to von Frey monofilaments. After L5 VRT $(N=11)$ or sham-L5 VRT $(\mathrm{N}=5)$ in $\mathrm{C} 57 \mathrm{BL} / 6$ mice, no effect on the withdrawal thresholds could be observed. The $\mathrm{CCl}$ in C57BL/6 mice $(N=11)$ resulted in a significant decrease of the withdrawal threshold of the ipsilateral paw compared to pre-nerve injury $(A)$. After L5 VRT $(N=10)$ or sham-L5 VRT $(N=7)$ in 129 J mice, no effect on the withdrawal thresholds could be observed (B). No effect on the withdrawal thresholds of the ipsi- and contralateral paw could be observed in both strains after L5 VRT, sham-L5 VRT or CCI (C and D). ${ }^{*} p<0.05$ compared to pre-nerve injury for $\mathrm{CCl}$ mice.

As for the effect of the L5 VRT on the withdrawal threshold of the contralateral paw in C57BL/6 and 129J mice, no decrease in PWTs could be observed (Figure 2.1C and D). Although a modest decrease of the PWT of the contralateral paw of the 129) mice could be noted at DPO $73.7 \pm 0.2(0.7 \pm 0.1 \mathrm{~g})$, this was not statistically different compared to pre-nerve injury PWTs $4.0 \pm 0.1(1.0 \pm 0.2 \mathrm{~g})(p$ $>0.05)$ (Figure 2.1D). The sham-VRT in the C57/BL6 $(N=5)$ and 129J $(N=7)$ did not result in any changes of the contralateral PWT (Figure 2.1C and D). No changes of the contralateral PWT of the $\mathrm{CCl}$ animals were noted (Figure 2.1D).

\section{Gait changes (CatWalk)}

All CatWalk parameters of the ipsilateral paw and contralateral paw were comparable at pre-nerve injury (Figure 2.2). 

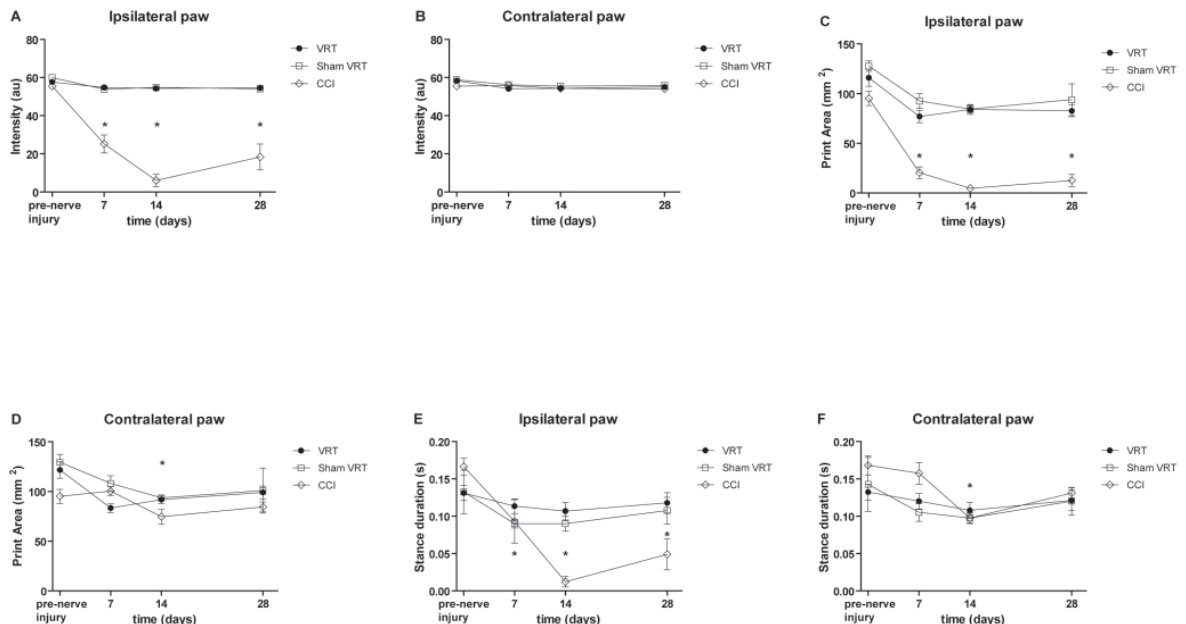

Figure 2.2. CatWalk parameters Intensity (A and B), Print Area (C and D) and Stance duration (E and F) after CCI $(N=11)$, L5 VRT $(N=11)$ and sham-L5 VRT $(N=5)$ in C57BL/6 mice. The Intensity, Print Area and Stance duration of the ipsilateral paw of mice with $\mathrm{CCl}$ showed a decrease from DPO 7 compared to pre-nerve injury and remained decreased up to DPO 28 (A, C and E). The Intensity and Print Area of the ipsilateral paw of the L5 VRT mice showed a significant decrease over time (A and C), however, the sham-L5 VRT resulted in a comparable decrease with respect to the same parameters (A and C). The L5 VRT or sham-L5 VRT showed no effect on the Stance duration of the ipsilateral paw (E). The Print Area and Stance duration of the contralateral paw of $\mathrm{CCI}$ mice were significantly decreased at DPO 14 (D and F). The Intensity and Print Area of the contralateral paw of the L5 VRT mice showed a significant decrease over time, however, the sham-L5 VRT resulted in a comparable decrease in the same parameters (B and D). A L5 VRT or sham-L5 VRT had no effect on the Stance duration of the contralateral paw $(\mathrm{F}) .{ }^{*} p<0.05$ compared to pre-nerve injury for $\mathrm{CCl}$ mice.

The Intensity, Print Area and Stance duration of the ipsilateral paw all significantly decreased after $\mathrm{CCl}$, with a maximum decrease at DPO 14 (Figure 2.2A, C and E). No significant decrease in CatWalk parameters could be observed in the contralateral paw after $\mathrm{CCl}$, with the exception of the Stance duration, which was significantly decreased at DPO 14 compared to pre-nerve injury (Figure 2.2B, D and $\mathrm{F}$ ).

The effect of a L5 VRT was compared to that of a L5 Sham-VRT. The L5 VRT or L5 Sham-VRT in C57BL/6 mice both had an effect over time. Intensity [time: F3,33 = $16.8, p<0.01$ ] and Print Area [time: F3,33 $=10.2, p<0.01$ ] of the ipsilateral paw were both significantly decreased, however no difference could be observed between the L5 VRT and L5 Sham-VRT (Intensity [intervention: F1,11 $=0.4, p=$ 0.5 ] and Print Area [intervention: F1,11 $=2.26, p=0.16$ ]) (Figure 2.2A and C). 
The L5 VRT affected the contralateral paw where the Intensity [time: F3,33 = 8.4, $p<0.01$ ] and Print Area [time: F3,33 $=6.1, p<0.01$ ] were significantly decreased but again, no difference between VRT and Sham-VRT could be observed (Intensity [intervention: F1,11 $=4.0, p=0.07$ ] and Print Area [intervention: $\mathrm{F} 1,11=2.23, p=0.16$ ]) (Figure 2.2B and D). The stance duration of the ipsi- and contralateral paw were unaffected by the L5 VRT or L5 Sham-VRT (Figure 2.2E and F).

To examine the effect of mouse strain on pain behaviour, we compared the effect of the L5 VRT in C57BL/6 and 129J mice. In both strains, the L5 VRT resulted in a significant time effect with respect to the Intensity of the ipsilateral [time: F3, $45=$ 3.1, $p<0.05$ ] as well as the contralateral paw [time: F3,45 $=2.9, p<0.05$ ] Although an effect over time was observed, no strain effect could be observed for the Intensity for both the ipsilateral [strain: $\mathrm{F} 1,15=0.2, p=0.7$ ] and contralateral paw [strain: F1,15 =0.1, $p=0.8$ ]) (Figure 2.3A and B).
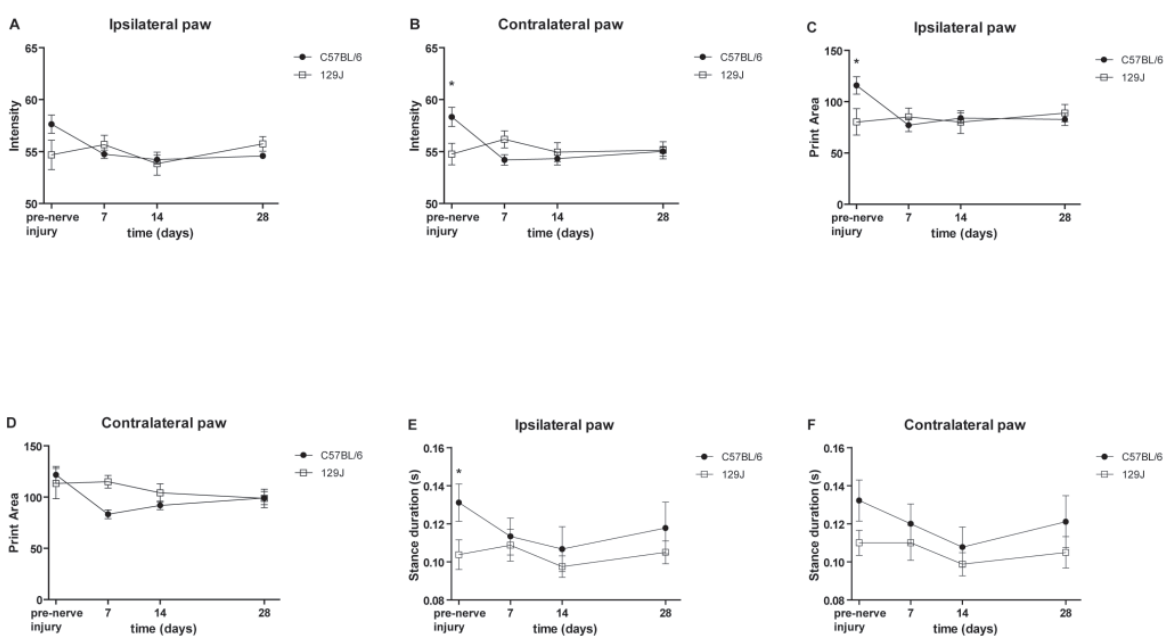

Figure 2.3. Effect of a L5 VRT in C57BL/6 $(N=11)$ and 129J $(N=10)$ on the CatWalk parameters Intensity, Print Area and Stance duration. For both 129J and C57BL/6 mice, L5 VRT resulted in an effect over time on the Intensity of the ipsi- and contralateral paw. However no difference between strains was observed (A and B). None of the other CatWalk parameters were affected of the ipsi- and contralateral paw of both strains $(C$ and $D)$. The Intensity of the contralateral paw at pre-nerve injury of the C57BL/6 mice was significantly increased compared to the 129J mice (B). Furthermore, the Print Area and Stance duration of the ipsilateral paw after a L5 VRT were significantly increased in the C57BL/6 mice as compared to 129J mice (C and D). ${ }^{*} p<0.05$ between C57BL/6 and 129J mice.

The Print Area and Stance duration of the ipsi- and contralateral paw did not show a time or strain effect as a result from the L5 VRT (Figure 2.3C-F). Pre-nerve 
injury values of the Intensity of the contralateral paw of the C57BL/6 $(58.3 \pm 0.9$ $\mathrm{au})$ and $129 \mathrm{~J}(54.8 \pm 1.0)$ were statistically different $(p<0.05)$. The L5 VRT did not affect the Print Area of the ipsilateral paw $\left(\mathrm{C} 57 \mathrm{BL} / 6115.9 \pm 8.6 \mathrm{~mm}^{2}\right)$ and (129) $\left.80.2 \pm 12.9 \mathrm{~mm}^{2}\right)(p<0.05)$ and the Stance duration $(\mathrm{C} 57 \mathrm{BL} / 60.13 \pm 0.01$ sec) and (129J $0.1 \pm 0.01 \mathrm{sec})(p<0.05)$ (Figure 2.3A, C and E).

\section{Discussion}

In the present study we demonstrated that the L5 VRT in C57BL/6 as well as in 129j mice did not result in the development of mechanical hypersensitivity as measured with the von Frey. Even changes in gait as measured with the CatWalk, suggested to be indicative for pain behavior, were not observed after the L5 VRT in both mouse strains. Our $\mathrm{CCl}$ control group however displayed behavioral signs of neuropathic pain using both the von Frey and CatWalk. Our observations in mice are not in line with those reported in rats [107], in which bilateral long lasting mechanical allodynia was observed. In rats, sectioning of the L5 efferent motor nerve resulted in a massive inflammation response and Wallerian degeneration, as seen by abundant infiltration of macrophages in the L5 ventral root, DRG and sciatic nerve. This inflammation is thought to "prime" uninjured adjacent sensory neurons, resulting in mechanical allodynia and thermal hyperalgesia $[107,240]$. The critical role of Wallerian degeneration in the induction of neuropathic pain was shown in mice (C57BL/Wld) with delayed Wallerian degeneration $[119,120]$. These animals presented with a reduced thermal hyperalgesia compared to normal C57BL/6 animals after CCI [149]. With respect to mechanical allodynia, no difference in the onset could be observed between $\mathrm{C} 57 \mathrm{BL} / 6$ and $\mathrm{C} 57 \mathrm{BL} / \mathrm{Wld}$ animals, although mechanical allodynia remained present for an extended period of time in C57BL/Wld mice [199], indicating a different role for Wallerian degeneration in the onset of thermal hyperalgesia and mechanical allodynia. In a recent publication, C57BL/6 mice displayed an over- expression of the tetraodontoxine-sensitive sodium channels Nav1.3 and Nav1.8 in the L4 and L5 DRG after L5 VRT [72], while L5 Dorsal Root Transection (DRT) in the rat fails to do so [13]. This over-expression of these sodium channels is thought to be critical for the development of neuropathic pain $[11,52,103]$. Furthermore, L5 VRT in rats results in activation of microglia and p38 MAPK (mitogen-activated protein kinase) which has been shown to be involved in the development of neuropathic pain [83], in both L4 and L5 DRGs. From this it was suggested that motor fiber injury might initiate neuropathic pain through uninjured primary afferents, which sequentially activate spinal microglia, leading to central sensitization. The central sensitization may contribute to the mechanical allodynia and thermal hyperalgesia produced by L5 VRT in rats. Despite the biochemical changes reported after a L5 VRT in rats and mice [72], in the present study we did not observe any changes in pain related behavior in the C57BL/6 and the more sensitive 129J mouse using two behavioral analysis. 
Indeed, biochemical changes are not a prerequisite for changes in pain related behavior. For instance, in Nav1.3 knockdown mice, neuropathic pain developed normally [150] and double knockouts of both Nav1.7 and Nav1.8 did not affect the development of neuropathic pain [114,151], thereby suggesting that upregulation of these channels is not a prerequisite for behavioral signs of neuropathic pain. Furthermore, DRG Nav1.8 was strongly downregulated [161] and no upregulation of Nav1.3 or correlate with pain behavior was observed after axotomy [162].

To exclude the possibility that the genetic background of our mice could be responsible for the absence of mechanical allodynia after a L5 VRT, we also used the more sensitive 129J strain. The 129J has been reported to be a very sensitive mouse strain to the induction of pain in a study on the effect of different measures of nociception in 11 mouse strains [144]. However also in the 129J mice, the L5 VRT did not result in the induction and maintenance of chronic neuropathic pain and the behavior was comparable to that of the C57BL/6 mice. Mogil and colleagues used different nociceptive assays, like hind limb denervation, carrageenan injection, formalin test and peripheral nerve injury. From this research, it was concluded that the 129J strain was more sensitive to peripheral nerve injury as compared to the C57BL/6 strain [144]. Interestingly, the same group recently reported that the percentage of maximal allodynia is almost comparable between C57BL/6 and 129 mice after a spared nerve injury [143]. In a model of lumbar radiculopathy, it was reported that the 129J mice had distinctly more tactile allodynia compared to other strains tested [102]. On the other hand, this distinction was not as clear in paclitaxel-induced neuropathic hypersensitivity [195]. These results indicate that beside strain, the pain model and testing methods used could also influence the outcome.

Furthermore, anatomical differences between rat and mouse may eventually underlie the observed differences in our study. The sciatic nerve in C57BL/6 mice predominantly receives fibers originating from the L3 and L4 spinal nerves [171], where the rat sciatic nerve receives the vast majority of its sensory fibers from the L4 and L5 spinal nerves [208]. True Blue labeling of the sciatic nerve in C57BL/7 mice revealed only a small percent (approximately $5 \%$ of total) of the L5 DRG was labeled [171]. This may implicate that a transection of the L5 ventral root in the mouse only affects a minority of the uninjured primary afferents and therefore does not result in behavioral signs of neuropathic pain. On the other hand, L5 VRT in rats resulted in upregulation of TNF- $\alpha$ in the ipsilateral L4 and L5 DRG [241], supposedly through projections of the L5 DRG to the L4 DRG. It was shown that after L5 spinal nerve transection in rats IB4 staining was not only reduced in the dorsal horn at the L5 level, but also in the dorsal horn of L4, suggesting the important contribution of L5 fibers to sensory neurons located in the L4 dorsal horn [190].

The transection of the L5 ventral root in C57BL/6 and 129J mice had no effect on the CatWalk parameters measured. The use of the CatWalk to measure pain 
related gait changes in animal models of pain was first suggested by Vrinten en Hamers [226]. They observed that in $\mathrm{CCl}$ operated rats, the time course of CatWalk changes paralleled that of von Frey measured mechanical allodynia. Since then, the use of the CatWalk for neuropathic pain models has been questioned based on the assumption that in most models damage to motor fibers is at least partially responsible for the observed gait changes $[143,164,216]$. With respect to our recent findings, the gait changes observed are probably the result of the surgery, rather than the selective injury of motor fibers or neuropathic pain, as the sham-VRT resulted in the same gait changes as seen in the L5 VRT. This is supported by the fact that the regularity index, a measure for interlimb coordination, was unaffected by the L5 VRT.

In summary, $\mathrm{CCl}$ in $\mathrm{C} 57 \mathrm{BL} / 6$ mice resulted in signs of tactile hypersensitivity. Indirect inflammation of intact sensory fibers due to the selective sectioning of the L5 motor fibers in C57BL/6 and 129J mice did not result in increased response to von Frey induced tactile stimulation or changes in gait as measured by the CatWalk. 


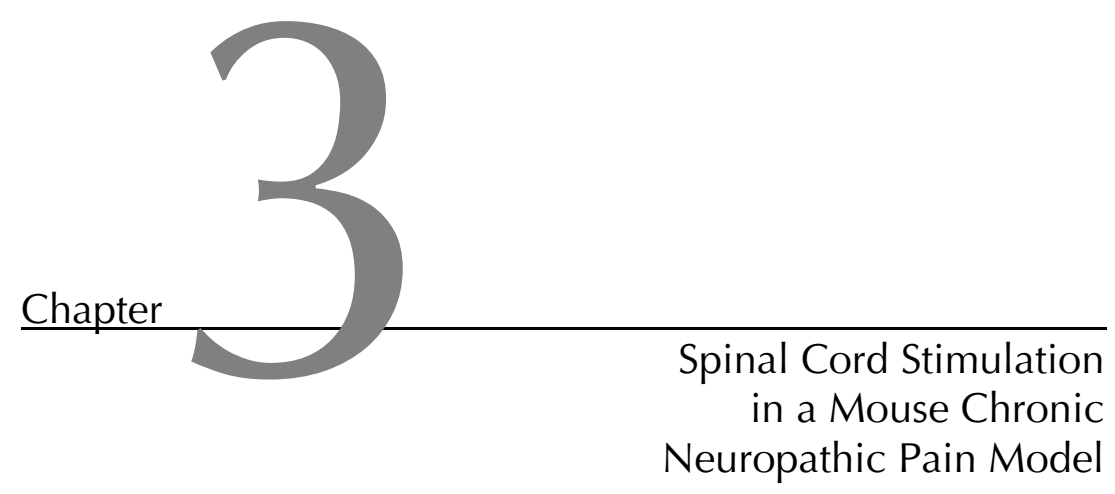

M. Truin

P. van Venrooij

V. Duysens

R. Deumens

M. van Kleef

E.A.J. Joosten

Neuromodulation 2007, 10(4): 358-362 


\section{Abstract}

Objective

Development of a spinal cord stimulation (SCS) system in a mouse model of chronic neuropathic pain.

\section{Materials and Methods}

Male C57BL/6 mice $(N=6)$ underwent a partial ligation of the sciatic nerve. Development of mechanical hyperalgesia was tested using the withdrawal response to tactile stimuli with the von Frey test. An SCS system was implanted on day 14 . On day 16 , the mice were stimulated for $30 \mathrm{~min}(\mathrm{f}=50 \mathrm{~Hz}$; pulse width $0.2 \mathrm{~ms}$ and stimulation at 2/3 of motor threshold). Repeated measure analysis of variance (ANOVA) and paired Student's t-test with Bonferroni correction were used to evaluate the development of mechanical hyperalgesia and the therapeutic effect of SCS.

\section{Results}

Five out of six mice developed marked mechanical hyperalgesia in the nervelesioned paw that persisted for the duration of the study (16 days). No changes contralateral to the injury were observed. In four out of five mice a successful implantation of the electrodes followed by stimulation was achieved. Then, SCS resulted in a fast and robust increase of withdrawal threshold back to pre-injury levels. After termination of the SCS, the withdrawal threshold of the ipsilateral paw slowly decreased. No effect of SCS on the contralateral paw was noted.

\section{Conclusion}

The development of a mouse SCS system is described that is practical in use, is reproducible and shows a comparative therapeutic effect in treatment of chronic neuropathic pain as reported in rat. 


\section{Introduction}

Spinal Cord Stimulation (SCS) has been used as an established treatment for chronic pain for almost 40 years now. Shealy and coworkers were the first to electrically stimulate the spinal cord dorsal columns to treat chronic, intractable pain in humans [188]. Presently more than 14,000 new systems for SCS are implanted every year. From then until now, the knowledge of the exact mechanisms by which SCS acts upon chronic pain is limited.

To improve insights about the mechanisms underlying chronic pain and SCS, experimental animal models are needed. The most commonly used animal models for chronic neuropathic pain are based on injury to the sciatic nerve in the rat. Peripheral nerve injury leads to profound behavioral changes in sensory functions [9]. A frequently used experimental model used for peripheral nerve injury is the partial ligation of the sciatic nerve according to Seltzer [187] that is known to result in profound chronic neuropathic pain (ie, allodynia, spontaneous pain). In Seltzer-injured rats, the onset of the tactile allodynia is rapid (one to three days) and persists for several weeks [187].

With respect to experimental SCS in treatment of chronic neuropathic pain mainly the Seltzer type, or partial sciatic nerve injury, is used [112,134]. In Seltzerinjured rats, SCS results in a robust and fast increase of the von Frey withdrawal threshold of the injured limb that is still noticeable $30 \mathrm{~min}$ after SCS cessation [197]. Interestingly, the spared nerve injury also has been reported recently to be a good experimental model in testing the effects of SCS in the rat [106].

In order to make further steps toward understanding molecular mechanisms involved in SCS after chronic neuropathic pain, an experimental model utilizing the mouse could be of great use. A major future advantage for studying SCS in the mouse is that it will allow studies at the molecular level based on genetically modified animals. Hence, the main aim of the present investigation was to develop an experimental mouse model of chronic neuropathic pain and SCS.

As in the rat, a tight ligation of $1 / 3$ and $1 / 2$ of the diameter of the sciatic nerve (Seltzer lesion) was reported to induce chronic neuropathic pain in the male C57BL/6 mouse [125]. The partial nerve injury markedly decreased paw withdrawal thresholds to both thermal and mechanical stimuli on the injured side. Whereas the thermal allodynia resolved by 49 days, the mechanical allodynia persisted for 70 days [125]. Based on these data, it was concluded that the partial ligation in the $\mathrm{C} 57 \mathrm{BL} / 6$ mouse sciatic nerve is a valuable model, designed to examine the biochemical basis of neuropathic pain in mice with specific gene modifications. In the present investigation, we intended to extend this model for analysis of SCS in neuropathic pain. 


\section{Animals}

For all experiments, a total of six male C57BL/6 mice (Charles River), weighing 20-25 g, were used. After ligation of the sciatic nerve the mice were housed individually. Housing was kept at a constant room temperature of $21 \pm 2{ }^{\circ} \mathrm{C}$ and under a standard $12 \mathrm{~h}$ normal light:dark cycle. Water and food pellets were available ad libitum. The wellbeing of the animals was monitored daily and documented by the responsible researcher (M. Truin).

\section{Material and Methods}

\section{General}

Animal experiments were performed in accordance with the European Directive for the Protection of Vertebrate Animals Used for Experimental and Other Scientific Purposes (86/609/EU) and were approved by the Committee for Experiments on Animals of Maastricht, Maastricht, The Netherlands. Surgery was performed under aseptic conditions in anesthetized animals. General anesthesia was induced by isoflurane inhalation (4\% in a 1:1 mixture with air, flow 400 $\mathrm{ml} / \mathrm{min}$ ) in a Plexiglas induction chamber. Anesthesia was maintained with an univentor $400 \AA$ injection system vaporizer (Zevenaar, The Netherlands) with an open mask system (Fluovac, Harvard Apparatus, Holliston, MA, USA) using $2 \%$ isoflurane in a 1:1 mixture with air at a flow of $250 \mathrm{ml} / \mathrm{min}$. Loss of body temperature was prevented by placing the animal on a automated heating pad $\left(37.5 \pm 0.5^{\circ} \mathrm{C}\right)$, (IDEE, Maastricht, The Netherlands).

\section{Unilateral Ligation of the Sciatic Nerve}

The unilateral sciatic nerve ligation was performed similar to the approach described in rats by Seltzer et al. (1990) [187]. In short, we used an 8/0 nonabsorbable silk suture with a reverse cutting and $3 / 8$ curve micro needle to ligate approximately $1 / 3$ to $1 / 2$ of the diameter of the left sciatic nerve. After the ligation, the wound was closed intracutaneously with a 5/0 silk suture. After surgery the mice were checked every day, for two days, to assure sutures were still in place.

\section{Implantation of the Spinal Cord Stimulation (SCS) Device}

The procedure for implanting the electrodes in the mice is comparable of that used in the rat $[33,36,112,197]$. Mainly because of the smaller size of the mice, adjustments to the materials had to be made (Medtronic Bakken Research Center BV, Maastricht, The Netherlands). The rectangular cathode (90/10 Platinum Iridium Alloy) used in the rats as described by Smits [197] was $3.00 \times 1.00 \mathrm{x}$ $0.025 \mathrm{~mm}$ and is now reduced to $2.25 \times 0.76 \times 0.025 \mathrm{~mm}$. The circular anode 
(90/10 Platinum Iridium Alloy) remained the same size (diameter of $5.80 \mathrm{~mm}$ and thickness of $0.127 \mathrm{~mm}$ ). Both leads, comprising insulated stainless steel wires and cathode/anode, were shortened to $5 \mathrm{~cm}$ as compared to the unipolar rat SCS leads which were $10 \mathrm{~cm}$. Furthermore, the two separate unipolar cathode and anode rat SCS leads with two individual unipolar connectors were replaced by one bipolar mouse SCS lead featuring one small bipolar connector (Figure 3.1).

Under general anesthesia, a laminectomy was performed at the level of T13. To identify T13, we used anatomical reference points as guidance. T12 is located at the apex of the curvature of the thorax of the mouse. Going caudally, we searched for the smallest processus spinosus. This processus spinosus is caudally marked by a prominent processus spinosus, the processus spinosus of L2. Then, the T13 vertebral body is located two segments rostral from the L2 processus. The 13th rib also was used as an anatomic landmark.

After the identification of T13, a small hole was made in the center of the lamina as rostrally as possible making sure not to penetrate the dura. After the introduction of the cathode into the epidural space, the lead was glued to the lamina of T12 using hystoacryl glue (Histoacryl ${ }^{\circledR}$, Braun, Melsungen, Germany) to prevent movement of the cathode. The anode was placed subcutaneously, as low in the abdominal wall as possible. The leads were then positioned subcutaneously, in a way that the leads could move freely without putting stress on the leads.

Next, a small incision was made at the base of the skull of the mice to tunnel the bipolar connector, including the leads subcutaneously to the incision site. The connector was then firmly cemented to the base of the skull using bone cement (Paladur®, Kulzer. Wehrheim, Germany). The periosteum of the bone was carefully removed to ensure good adhesion of the connector.

Animals were allowed to recuperate after the surgery for 48 hours, before being submitted to the SCS. We used the Grass S88 stimulator (Astro-Med, Grass Instruments, Warwick, RI, USA) fitted with a Grass SIU-5 stimulus isolator and a Grass constant current unit. The swivel (ensuring free movement of the wire connecting the SCS device and the connector) was also adjusted to minimize drag. The wire itself was improved compared to the wire used with rats $(9.1 \mathrm{~g})$, by making it lighter $(3.1 \mathrm{~g})$. The stimulus paradigm was as follows: first the motor threshold was assessed (frequency $2 \mathrm{~Hz}$, pulse width $0.2 \mathrm{~ms}$ ) and the actual stimulation was done at 2/3 of the motor threshold (frequency $50 \mathrm{~Hz}$, pulse width $0.2 \mathrm{~ms}$ ). Stimulation was maintained for $30 \mathrm{~min}$. During stimulation, the mice could move freely and did not show discomfort due to the stimulation. These settings were similar to those used in the rat experiments [197].

\section{Behavioral testing}

Before partially ligating the sciatic nerve, each mouse was tested for the behavioral response to mechanical stimuli, using the von Frey test. This is the baseline withdrawal threshold. After the ligation of the sciatic nerve, the 
withdrawal threshold was tested on day 2, 7 and 14 (days postoperative [DPO] 2, 7 and 14). Testing took place in a set of Plexiglas cages, each measuring $6.5 \times 7.5$ $X 8.0 \mathrm{~cm}$, with a wire mesh floor. We allowed the animals to habituate for 60 minutes. After acclimatization, the von Frey filaments were applied, in an order of increasing stiffness, through the wire mesh floor to the mid plantar surface of the right and left hind paw until the filaments bent slightly. We scored a positive withdrawal when the animal showed a response (brisk withdrawal) to three out of the five stimuli presented. The animals were tested using the von Frey monofilaments (North Coast Medical Inc, Morgan Hill, Ca, USA) 1.65, 2.44, 2.83, $3.22,3.61,3.84,4.08,4.17,4.31$ and 4.56 (corresponding to 0.008, 0.04, 0.07, $0.16,0.4,0.6,1.0,1.4,2.0$, and $4.0 \mathrm{~g}$ ). Immediately before SCS the animals were tested once more using the von Frey filaments (pre-SCS). During stimulation the testing was repeated after $t=15,30,45$ and $60 \mathrm{~min}(\mathrm{t}=15,30,45$ and 60). Stimulation was ceased after $30 \mathrm{~min}$.

\section{Statistical Analysis}

The data are presented as mean \pm SEM. For statistical analysis of differences in withdrawal thresholds over time, we used repeated measure analysis of variance (ANOVA). We compared each individual time point after baseline withdrawal threshold with that of the baseline withdrawal threshold using the paired Student's $t$-test. Normally, $p<0.05$ is regarded as the level of significance. However, because multiple comparisons were made, the level of significance was adjusted (post hoc correction) to $p<0.05 / 3=p<0.016$. This was also true for the comparison of von Frey values between pre-SCS and $t=15,30,45$ and 60 ( $p<$ $0.05 / 4=p<0.0125)$. Furthermore, the paired Student's $t$-test was used comparing the ipsilateral paw with the contralateral paw.

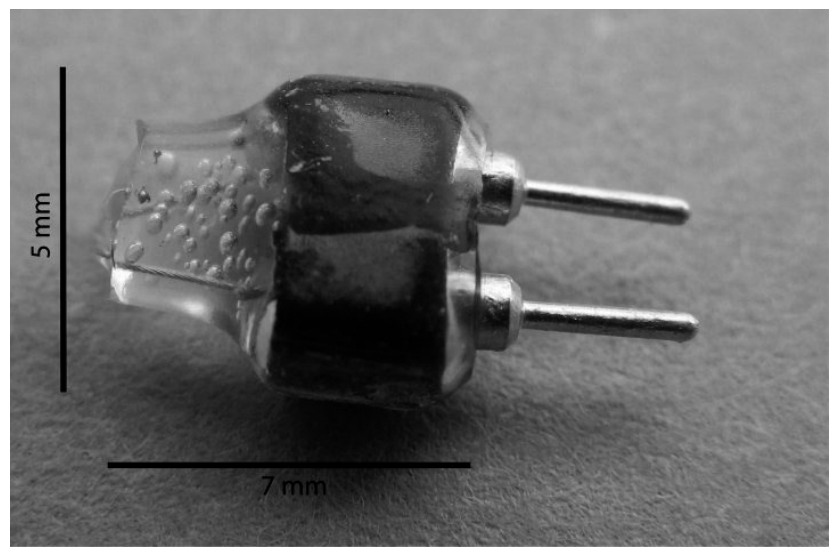

Figure 3.1. The bipolar connector $(7 \times 5 \times 2 \mathrm{~mm})$ used to place on the base of head of the mouse. The silicon coating prevents contact between the cathode and anode. 


\section{Results}

\section{General Behavior}

After the ligation of the left sciatic nerve, the mice displayed normal behavior. They could climb in the cage, hang upside down and stand on their hind legs. Body weight decreased 2-4\% after the partial ligation but returned to pre-injury values within five days. The weight loss after implantation of the electrodes was more severe: between $5 \%$ and $10 \%$. Therefore, Ringer's lactate buffer $(\mathrm{pH} 7.0)$ was administered subcutaneously (1-1.5 cc) during and immediately after surgery. This resulted in a significant reduction of weight loss. No autotomy was observed.

\section{Total Population}

Data with respect to the withdrawal response to tactile stimuli (von Frey) were only included if recordings of all time points were tested (ie, baseline, DPO2, DPO7, DPO14, pre-SCS, SCS-15, SCS-30, 45 and $60 \mathrm{~min}$ ). Finally, four out of the six animals were included. We excluded one animal because no withdrawal response could be elicited from the ipsilateral paw after ligation of the left sciatic nerve (ie, non-responder to injury). One animal could not be used for analysis, because it was in poor condition after the implantation of the SCS device. The four animals that received SCS all showed a marked increase in the withdrawal threshold of the ipsilateral paw.

\section{Development of Mechanical Hyperalgesia}

Following the partial ligation of the sciatic nerve, the withdrawal threshold of the ipsilateral paw to tactile stimuli showed a statistically significant decrease at DPO2 compared to pre-injury or baseline, $0.13 \pm 0.09 \mathrm{~g}$ and $1.00 \pm 0.0 \mathrm{~g}$, respectively (t-test, $p<0.002$ ) (Figure 3.2A). This decrease in withdrawal threshold persisted for at least two weeks. No change in withdrawal threshold to tactile stimuli of the contralateral paw was observed at any time (Figure 3.2A).

\section{Spinal Cord Stimulation}

After establishing the motor threshold $(0.73 \pm 0.08 \mathrm{~mA}$ [mean $\pm \mathrm{SEM}])$, the stimulation current was reduced to $2 / 3$ of the motor threshold $(0.5 \pm 0.05 \mathrm{~mA}$ [mean $\pm \mathrm{SEM}])$. To ensure that the implantation of the electrode did not affect the withdrawal threshold, von Frey values of DPO14 and DPO16 were compared: No significant difference was noted between pre-implantation at DPO14 $(0.22 \pm 0.13$ $\mathrm{g}[$ mean $\pm \mathrm{SEM}])$ and after the implantation of the electrodes at DPO $16(0.08 \pm$ $0.03 \mathrm{~g}$ [mean $\pm \mathrm{SEM}]$ ) (Student's $t$-test; $p<0.26$ ).

As can be deduced from Figure 3.2B, a 30-min SCS has a significant effect on the withdrawal threshold to von Frey stimuli of the ipsilateral paw: already 15 min after initiation of SCS the withdrawal threshold of the injured paw increased to $0.85 \pm 0.22$ g. (mean \pm SEM) However, no statistical significance was reached ( $t$ - 
test compared with pre-SCS, $p<0.035)$. Thirty minutes after the initiation of SCS, a significant increase in withdrawal threshold was observed, which even returned to pre-neuropathy levels: mean withdrawal level of the ipsilateral paw was $1.10 \pm$ $0.10 \mathrm{~g}$ (mean \pm SEM) ( $t$-test compared with pre-SCS, $p<0.0024)$.

After termination of the SCS, the withdrawal threshold of the ipsilateral paw slowly decreased to $0.50 \pm 0.06 \mathrm{~g}$ (mean $\pm \mathrm{SEM}$ ) at $60 \mathrm{~min}$ ( $t$-test compared with pre-SCS, $p<0.011$ ). No effect of the SCS on the withdrawal threshold of the contralateral paw was noted during the experiment (Figure 3.2B).
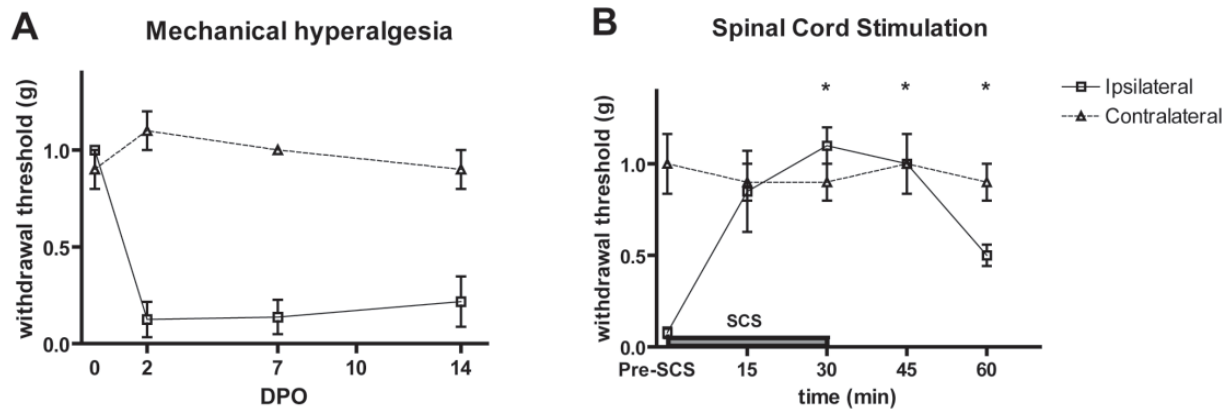

Figure 3.2. (A) All data are presented as mean \pm SEM. Decrease in withdrawal threshold of ipsilateral hind paw to mechanical stimuli (von Frey) following the Seltzer lesion $(N=4)$. No change in withdrawal threshold of the contralateral paw was observed. (B) SCS $(N=4)$ resulted in a rapid increase of the mean withdrawal threshold. After 15 minutes of stimulation withdrawal threshold reached $0.85 \pm 0.22 \mathrm{~g}$. Maximum stimulation effect to pre-neuropathy level was achieved after 30 min, which was statistically different compared to pre-SCS values $(p<0.0024)$. Statistical significance $(p<0.0125)$ is indicated with asterisks. After cessation of SCS, withdrawal threshold gradually decreased to $0.50 \pm 0.06 \mathrm{~g}$. SCS did not affect the withdrawal threshold of the contralateral paw.

\section{Discussion}

In the present study, we demonstrate and confirm that the onset, progression, and duration of the changes in withdrawal response to tactile stimuli induced by a partial nerve injury evoked pain condition in the $\mathrm{C} 57 \mathrm{BL} / 6$ strain of mouse are similar to those previously reported in rats and mice $[125,187]$. A new finding is that 30 minutes of SCS ( $\mathrm{f}=50 \mathrm{~Hz}$, pulse width $2 \mathrm{msec}$.) induced a rapid and robust increase in the withdrawal threshold to tactile stimulation of the ipsilateral paw of the $\mathrm{C} 57 \mathrm{BL} / 6$ male mouse. No changes contralateral to the nerve injury were noted. Thirty minutes after initiating SCS, a significant increase in the withdrawal threshold to tactile stimuli in the $\mathrm{C} 57 \mathrm{BL} / 6$ mice was observed, which even returned to pre-neuropathy levels. In the Sprague-Dawley rat, a maximal pain relieving effect also was noted after $30 \mathrm{~min}$ of SCS, although pre-neuropathy levels were not reached $[33,36,206]$. However, in a recent study, a differential effect of SCS related to the severity of mechanical allodynia was reported: a return 
to pre-neuropathy levels was noted only for mild allodynic rats, whereas in severe allodynic male Sprague-Dawley rats SCS did not lead to a statistically significant increase of withdrawal thresholds to tactile stimuli [197]. Although in the present small-scale study SCS seems to be a more efficient therapy for mechanical hyperalgesia in chronic neuropathic $\mathrm{C} 57 \mathrm{BL} / 6$ mice than in rats, a large scale study needs to be performed in which mice will be subdivided into groups based on the level of allodynia similar to those previously described for the rat [197]. This is complicated, however, because already the pre-neuropathy levels in C57BL/6 mice significantly differ from the Sprague Dawley rat $(\mathrm{C} 57 \mathrm{BL} / 6$ mice $(1.0 \mathrm{~g})$ and Sprague Dawley rats $>60-70 \mathrm{~g}$, and assessed with the same von Frey equipment). Hence, a subdivision in $\mathrm{C} 57 \mathrm{BL} / 6$ mice with respect to the level of allodynia based on cut-off points in a consecutive order of filament stiffness as used in the rat will never result in identical subgroups.

To achieve maximal effect of the present SCS approach, it is important to stimulate at the correct location. In our opinion, this optimal location would be the position where the roots of the sciatic nerve leave the central nervous system at L3-L6. This corresponds with the vertebral body of T13. Although we cannot exclude the possibility that SCS of levels other than L3-L6 could be therapeutically beneficial, the present results indicate that SCS of L3-L6 has optimal effects (ie, withdrawal thresholds returning to baseline levels). Previously the localization of the electrode at T13 was verified in rat by X-ray [197]. Unfortunately, verification of the correct position of the electrode using X-ray was not possible in our mice. Attempts were made, but due to the lower bone density in the mouse as compared to the rat and the relative low resolution of the X-ray machine, they were unsuccessful. Therefore, verification of the position of the electrode in these mice was done retrospectively, after sacrificing the animals at the end of the experiment. Based on the anatomical landmarks (ie the vertebral body of T13), the correct localization of the electrode was verified.

It should be noted that various difficulties were encountered in developing this SCS model in mice. One of the major complications was the mobility of mice as compared to rats. Mice continuously scratch and groom which results in a relative high percentage of animals in which the plugs located in the animals neck were dislocated. To solve this problem, we developed one small bipolar connector that then needs to be firmly cemented to the base of the skull.

Again, the mobility of the mice formed a complication in the correct assessment of behavioral changes as measured with the von Frey. Whereas rats can be assessed for tactile allodynia after a habituation of only $10 \mathrm{~min}$ [197], mice need to habituate to the von Frey cages for at least $60 \mathrm{~min}$. Consequently, SCS was performed while animals were located within the von Frey test cage. Then, appropriate and correct assessment of tactile allodynia in these mice was possible. 


\section{Conclusions}

We demonstrate that SCS in the mouse is technically possible and results in a fast and significant pain relief in allodynic mice. It is our understanding that with the present developed SCS system in neuropathic mice it will be possible to study the underlying molecular mechanism of SCS and pain relief in genetically modified animals. 


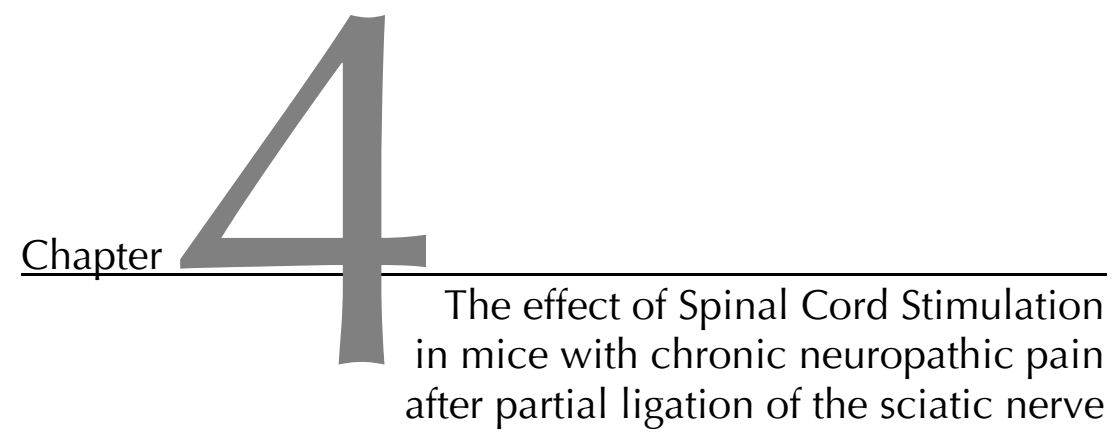

M. Truin

M. van Kleef

Y. Verboeket

R. Deumens

W. Honig

E.A.J. Joosten

Pain 2009, 145 (3): 312-318 


\section{Abstract}

\section{Background}

The effect of Spinal Cord Stimulation (SCS) in chronic neuropathic pain is inversely related to severity of mechanical allodynia and the underlying mechanisms are poorly understood. To understand these mechanisms further we aimed to develop a model of SCS in a neuropathic mouse. Further, the CatWalk analysis, which is claimed to be an improved test for mechanical allodynia and therapeutic intervention, was used to analyze the effect of SCS on mechanical allodynia.

\section{Methods}

Male $\mathrm{C} 57 \mathrm{BL} / 6$ mice $(N=31)$ underwent partial ligation of the sciatic nerve. After 14 days an electrode was implanted and the effect of SCS $(N=22)$ on mechanical allodynia was tested. Unligated mice $(N=8)$ also received SCS. Behavioral analysis was performed using von Frey filaments and the CatWalk system.

\section{Results}

The withdrawal threshold showed a significant decrease which remained over time. Changes in CatWalk parameters were observed after 2 days, but tended to diminish during the next 14 days. Thirty minutes of SCS resulted in a $100 \%$ response and return to pre-neuropathy levels of the withdrawal threshold. The effect of SCS on the withdrawal threshold was comparable for the most severe and milder allodynic animals. SCS did not affect any of the CatWalk parameters in all mice.

\section{Conclusion}

We developed a model of SCS in a chronic neuropathic pain C57BL/6 mouse. The CatWalk gait analysis does not result in detection of behavioral changes to SCS in mice with chronic neuropathic pain and control animals. This model allows future molecular-genetic studies on the mechanisms of SCS in chronic neuropathic pain. 


\section{Introduction}

Despite the use of Spinal Cord Stimulation (SCS) in the treatment of patients with chronic neuropathic pain $[85,87,98]$ understanding of the underlying mechanisms of its favorable effects is still relatively limited. In the experimental field, new insights have been obtained on mechanisms that could be responsible for the effect of SCS in chronic neuropathic pain models $[33,112,134]$. The most commonly used neuropathic pain model to study experimental SCS is the partial ligation of the sciatic nerve or Seltzer model [187]. The Seltzer ligation results in a fast and pronounced development of mechanical allodynia (as assessed with von Frey filaments) and thermal hyperalgesia in rats and this persists for more than 7 months [187].

In Seltzer injured rats, SCS results in a robust and fast increase in the von Frey withdrawal threshold of the injured limb which is still noticeable 60 min after cessation of SCS [197]. Similar to the clinical situation, $30-50 \%$ of rats with chronic neuropathic pain do not respond adequately to SCS $[36,97,206]$. Moreover, an inverse relationship of the degree of mechanical allodynia and the effect of SCS was shown [197]. From these experimental data, it was suggested that the selection and subdivision of patient groups similar to those defined in the rat model [197] may provide better pre-treatment prediction of possible therapeutic benefits of SCS. Indeed, after the analysis of various prognostic factors in 36 CRPS-I patients treated with SCS, the degree of brush-evoked allodynia was shown to be a prognostic factor of SCS treatment outcome [222].

To analyze the underlying mechanisms of SCS in experimental chronic neuropathic pain further it might be important to develop an SCS model in mice with chronic neuropathic pain. An SCS mouse model might allow us to study the molecular-genetic mechanisms of neuropathic pain and SCS, using mice with specific gene modifications. In a pilot experiment we have already described the technical requirements and aspects to apply SCS in chronic neuropathic C57BL/6 mice [217]. It has been reported that C57BL/6 Seltzer injured mice develop mechanical allodynia [125] similar to that described in the Sprague-Dawley rat $[134,187]$.

Whereas testing of mechanical allodynia using the von Frey withdrawal threshold is presently used as the gold standard, another method, the CatWalk gait analysis is additionally used in experimental pain research. The CatWalk is a fully automated objective gait analysis and was initially developed to analyze gait changes after spinal cord injury in rats [66]. In addition, the CatWalk has been suggested as a novel rapid and highly objective alternative to the von Frey method for measuring mechanical allodynia in rat animal models of chronic neuropathic pain [226] or acute inflammatory pain [58].

Therefore, the aim of this investigation was twofold: first to study the effect of SCS on mechanical allodynia in the Seltzer injured C57BL/6 mouse using the von Frey 
assessment and second to implement the CatWalk gait analysis for detection of pain-related gait changes after SCS in mice with chronic neuropathic pain.

\section{Methods}

\section{Animals}

In this experiment a total of 39 male C57BL/6 mice (Charles River), weighing 20$25 \mathrm{~g}$, were used. In 31 mice, the sciatic nerve was ligated and 8 mice served as controls, only receiving SCS. Housing was kept at a constant room temperature of $21 \pm 2{ }^{\circ} \mathrm{C}$ and humidity $(55 \pm 15 \%$ ) under a normal 12:12 h light/dark cycle. Water and food were available ad libitum. The well-being of the animals was monitored daily and documented by the responsible researcher.

\section{General}

Animal experiments were performed in accordance with the European Directive for the Protection of Vertebrate Animals Used for Experimental and Other Scientific Purposes (86/609/EU) and were approved by the Committee for Experiments on Animals of Maastricht, The Netherlands. Surgery was performed under aseptic conditions in anesthetized animals. General anesthesia was induced by isoflurane inhalation ( $4 \%$ in a 1:1 mixture with air, flow $400 \mathrm{ml} / \mathrm{min}$ ) in a Plexiglas induction chamber. Anesthesia was maintained with an univentor 400® injection system vaporizer (Zevenaar, Holland) with an open mask system (Fluovac, Harvard Apparatus) using $2 \%$ isoflurane in a 1:1 mixture with air flow of $250 \mathrm{ml} / \mathrm{min}$. Loss of body temperature was prevented by placing the animal on a automated heating pad $\left(37.5 \pm 0.5^{\circ} \mathrm{C}\right)$, (IDEE, University of Maastricht).

Animals that showed signs of extreme weight loss (> $15 \%$ of bodyweight at delivery), cachectic behaviour, or who did not groom, were euthanized.

\section{Unilateral ligation of the sciatic nerve}

A unilateral sciatic nerve ligation was performed similar to the approach described in rats by Seltzer [187] and Malmberg and Basbaum [125]. In short, the left sciatic nerve was exposed by blunt dissection and carefully freed from surrounding connective tissue. Just distal to the posterior biceps semitendinosus, but proximal to the little fat pad that lies a few millimeters distal to this site, the ligation was performed. We used an $8 / 0$ non-absorbable silk suture with a reverse cutting and $3 / 8$ curve micro needle to ligate approximately $1 / 3-1 / 2$ of the diameter of the left sciatic nerve. After the ligation, the wound was closed intracutaneously with a 5/0 silk suture. After surgery the mice were inspected daily, to assure sutures were still in place. 


\section{Implantation of the Spinal Cord Stimulation (SCS) device}

The procedure for implanting the electrodes in the mice was done under general anesthesia. Fourteen days post-operatively (DPO 14) a laminectomy was performed at the level of T13. Using the 13th rib as an anatomical landmark to identify the processus spinosus of $\mathrm{T13}$, a small hole was made in the centre of the lamina as rostrally as possible making sure not to penetrate the dura. After the introduction of the cathode into the epidural space, the cathode was glued to the lamina of T12 using hystoacryl glue (Histoacryl $®$, Braun) in order to prevent movement of the lead. The anode was placed subcutaneously, in the abdominal wall as low as possible. The two leads were then positioned subcutaneously, in a way that they could move freely without putting stress on the leads.

Next a small incision was made at the base of the skull of the mice to tunnel the bipolar connector including the leads subcutaneously to the incision site. The periosteum of the bone was carefully removed to ensure good adhesion of the connector. The connector was then firmly cemented to the base of the skull using bone cement (Paladur ${ }^{\circledR}$, Kulzer).

Animals were allowed to recuperate after the surgery for $48 \mathrm{~h}$, before being submitted to the SCS. For SCS we used a Grass S88 stimulator (Astro-med Grass Warwick, USA) fitted with a Grass SIU-5 stimulus isolator and a Grass constant current unit. The stimulus paradigm was as follows: first the motor threshold was assessed (frequency $2 \mathrm{~Hz}$, pulse width $0.2 \mathrm{~ms}$ ) and the actual stimulation was performed at $2 / 3$ of the motor threshold (frequency $50 \mathrm{~Hz}$, pulse width $0.2 \mathrm{~ms}$ ). Spinal cord stimulation was maintained for $30 \mathrm{~min}$. During SCS, the mice could move freely. No signs of discomfort were noted due to the stimulation. The settings used for SCS were similar to those used by others in the rat [197] and mice [217].

To study the effect of SCS in control animals, electrodes were implanted into nonligated mice. These animals were also allowed to recuperate after the surgery for $48 \mathrm{~h}$, before being submitted to SCS.

\section{Behavioral testing}

von Frey withdrawal threshold to mechanical stimuli

Before partially ligating the sciatic nerve each mouse was tested for the behavioral response to mechanical stimuli, using the von Frey test. This is the baseline withdrawal threshold (pre-operation threshold). The control animals were tested before receiving the SCS implant (baseline).

After the ligation of the sciatic nerve, the withdrawal threshold was tested on 2, 7 and 14 days post-operatively. Mice that did not show a decrease in withdrawal threshold of more than one von Frey filament compared to baseline were excluded and we qualified them as "non-allodynic". Testing was done in a set of Plexiglas cages, each measuring $6.5 \times 7.5 \times 8.0 \mathrm{~cm}$, with a wire mesh floor. The animals were allowed to adapt for 60 minutes before testing. After 
acclimatization, the von Frey filaments were applied, in an order of increasing stiffness, through the wire mesh floor to the mid-plantar surface of the right or left hind paw until the filaments bent slightly. A positive withdrawal was scored when the animal showed a response (brisk withdrawal) to 3 out of the 5 stimuli presented. The animals were tested using 10 von Frey monofilaments (North Coast Medical Inc., Ca, USA) with logarithmically incremental stiffness (0.008, $0.04,0.07,0.16,0.4,0.6,1.0,1.4,2.0$, and $4.0 \mathrm{~g}$ ).

Immediately before SCS the animals were tested for the presence of mechanical allodynia using the von Frey filaments (pre-SCS). Von Frey analysis was done at several time points after the initiation of SCS $(t=15,30,45,60,75$ and 90 minutes). Spinal cord stimulation was ceased after $30 \mathrm{~min}$.

To study the effect of SCS related to the severity of mechanical allodynia, the allodynic mice were categorized based on their response to the various von Frey filaments just before SCS (pre-SCS) (Table 1).

\section{Catwalk gait analysis}

To exclude a possible effect of the stimulation of the hind paws by the von Frey filaments on CatWalk parameters, CatWalk data collection always was done on the same day and before the von Frey testing.

In general, mice cross the CatWalk runway easily and at a constant speed. CatWalk gait analysis was done on the same day as the von Frey test during the first 14 days of the experiment, or baseline for the control animals. During the SCS-protocol CatWalk data were obtained pre-SCS and after $30 \mathrm{~min}$ of stimulation and at $60 \mathrm{~min}$ after cessation of SCS. These time points were selected because a 15-min timeframe between von Frey assessments was too limited to allow us to collect appropriate CatWalk runs.

The CatWalk analysis has been described in detail $[66,96]$. In short, the CatWalk system consists of a glass walkway which contains light from a white fluorescent source. The light rays from this source are completely reflected internally. When an object touches the glass runway, the light is reflected downwards, where it is detected by a video camera (Sony 3CCD Color Video Camera; DXC-990/990P). This signal is then digitized, which allows analysis by the CatWalk program software [66]. With the CatWalk, a vast variety of static and dynamic gait parameters can be measured. Some of these parameters, including Mean intensity of paw placement, Stance duration and Swing duration of the hind paw, have been linked to mechanical allodynia and neuropathic pain [226]. Because the speed and consistency at which the animals cross the glass runway can affect various CatWalk parameters [96] a constant crossing time is a prerequisite for correct CatWalk analysis. 
In the present study the following individual paw parameters were used:

Mean intensity (expressed in arbitrary units, a.u): Mean intensity of the pixels forming the maximum area.

Stance duration (expressed in seconds): Time of contact of the paw with the glass floor.

Swing duration (expressed in seconds): Time that the paw is not in contact with the glass floor.

\section{Statistical Analysis}

The data are presented as mean \pm standard error of the mean (SEM). For statistical analysis of differences over time of the withdrawal thresholds and the CatWalk parameters over time, we used the non-parametric Friedman test, followed by a Dunn's post-hoc test. The Wilcoxon test was used to compare the withdrawal thresholds and CatWalk parameters between DPO14 and pre-SCS. For analysis of differences in weight and crossing time the repeated measure analysis of variance (ANOVA) with Bonferroni correction was used. $p<0.05$ was considered to be statistically significant.

\section{Results}

\section{General observations}

The majority of the operated mice appeared healthy and well groomed. None of the mice showed signs of autotomy after ligation of the left sciatic nerve. Paw gesture of the ipsilateral paw was slightly altered, but this did not interfere with the normal daily activities of the mice. Body weight slightly decreased after the partial ligation of the sciatic nerve but returned to pre-injury levels within 5 days. A total of 29 out of the 31 ligated animals could be used as they developed mechanical allodynia as assessed with von Frey filaments ( 2 animals were nonallodynic). For SCS 22 animals were used to study the effect on mechanical allodynia (2 animals were in poor health after implantation, in 3 animals the connector came loose from the cranium, and 2 animals did not show any reaction to the mechanical stimulation after implantation).

On the same 29 animals used for the von Frey analysis the CatWalk analysis of the gait during the development of mechanical allodynia was performed. To study the effect of SCS on gait we included 19 animals (3 additional animals were excluded compared to the von Frey analysis of SCS, because these animals were reluctant to cross the CatWalk runway.

A total of 7 out of 8 control animals could be used for von Frey and CatWalk analysis (one animal was excluded because the connector came loose). 


\begin{tabular}{rrrrr}
\hline $\begin{array}{r}\text { Pre-nerve injury } \\
\text { withdrawal } \\
\text { threshold }(\mathbf{g})\end{array}$ & $\begin{array}{r}\text { Pre-stimulation } \\
\text { withdrawal } \\
\text { threshold }(\mathbf{g})\end{array}$ & $\begin{array}{r}\text { Withdrawal } \\
\text { threshold after } \mathbf{3 0} \\
\text { min SCS }(\mathbf{g})\end{array}$ & $\begin{array}{r}\text { Percent of pre-nerve } \\
\text { injury withdrawal } \\
\text { threshold at SCS30 }\end{array}$ \\
\hline $1.0 \pm 0.40(\mathrm{n}=2)$ & 0.008 & $1.0 \pm 0.00$ & $119.05 \pm 47.76$ \\
$0.9 \pm 0.07(\mathrm{n}=6)$ & 0.02 & $0.9 \pm 0.07$ & $104.44 \pm 14.05$ \\
$1.0 \pm 0.00(\mathrm{n}=2)$ & 0.04 & $1.0 \pm 0.20$ & $120.00 \pm 20.06$ \\
& $1.0 \pm 0.63(\mathrm{n}=4)$ & 0.07 & $1.0 \pm 0.12$ & $133.33 \pm 33.33$ \\
& $0.9 \pm 0.08(\mathrm{n}=5)$ & 0.16 & $0.9 \pm 0.13$ & $108.00 \pm 7.99$ \\
\hline Mean & $1.5 \pm 0.29(\mathrm{n}=3)$ & 0.4 & $1.5 \pm 0.33$ & $90.48 \pm 0.00$ \\
\hline SEM & $\mathbf{1 . 0 3}$ & $\mathbf{0 . 1 1}$ & $\mathbf{1 . 0 8}$ & $\mathbf{1 1 1 . 3 4}$ \\
\hline
\end{tabular}

Table 4.1. Effect of SCS on the withdrawal threshold of the different "allodynic groups" ( $N=22)$. The first column depicts the absolute pre-nerve injury withdrawal threshold of the different "allodynic groups", defined by the degree of allodynia at pre-stimulation (Column 2). The third column depicts the absolute withdrawal threshold after $30 \mathrm{~min}$ of SCS. The last column depicts the effect of SCS expressed in percent of pre-nerve injury withdrawal threshold. No difference could be detected between the degree of allodynia and the response to SCS.

\section{Withdrawal threshold to mechanical stimuli after partial nerve injury}

A marked decrease in the withdrawal threshold of the ipsilateral paw at DPO2 compared to baseline was noted in Seltzer ligated animals $(N=29)$ (Figure 4.1A). Before the Seltzer operation, the mean withdrawal threshold of the ipsilateral paw of these mice were $1.00 \pm 0.05 \mathrm{~g}$. Two days after the ligation a reduction was observed to $0.16 \pm 0.03 \mathrm{~g}(p<0.001)$, which remained stable up to DPO14. The withdrawal threshold of the contralateral paw at baseline $(1.00 \pm 0.06 \mathrm{~g})$ was comparable to that of the ipsilateral paw $(1.00 \pm 0.05 \mathrm{~g})$. The partial ligation of the left sciatic nerve did not result in alterations in withdrawal threshold of the contralateral paw (Figure 4.1A).

\section{Withdrawal threshold to mechanical stimuli during and after Spinal Cord Stimulation}

A total of 29 allodynic animals were implanted with a SCS device at DPO14. Twenty-two received SCS. In this population of SCS responders $(N=22)$, the mean pre-operation withdrawal threshold (baseline) was $1.03 \pm 0.07 \mathrm{~g}$.

The mean pre-stimulation withdrawal threshold (pre-SCS) of the ipsilateral paw was $0.11 \pm 0.03 \mathrm{~g}$, which did not differ from the withdrawal threshold at DPO14 (pre-implantation) $(0.15 \pm 0.03 \mathrm{~g}, p>0.05)$.

SCS increased the mean withdrawal threshold to $0.78 \pm 0.08 \mathrm{~g}$ after $15 \mathrm{~min}$ of stimulation as compared to pre-SCS $(p<0.001)$ (Figure 4.1B). Complete return to 
pre-neuropathy levels $(1.08 \pm 0.06 g, p>0.05)$ of the withdrawal threshold was observed after 30 min of SCS (Figure 4.1B).

Thirty minutes after its initiation, SCS was terminated. The mean withdrawal threshold decreased to $0.26 \pm 0.04 \mathrm{~g}$ at $75 \mathrm{~min}$ and was not significantly different from that at pre-SCS $(p>0.05)$. During and after SCS no changes in withdrawal threshold of the contralateral paw were noted (Figure 4.1B).

No effect on the withdrawal threshold of both the ipsilateral and contralateral hind paw after implantation and SCS could be observed in the control animals (Figure 4.1C).

Based on their responses all allodynic animals were categorized to the various filaments after 16 days (pre-SCS) of chronic neuropathic pain (Table 4.1). The most "sensitive" animals responded to a filament of $0.008 \mathrm{~g}$ whereas the least "sensitive" animals responded to $0.4 \mathrm{~g}$. Pre-nerve injury withdrawal threshold of the ipsilateral paw of all mice did not differ (Table 4.1). As can be deduced from our data, the degree of mechanical allodynia is not related to the response to SCS. The most severely allodynic animals responded as well to SCS as the less allodynic animals in the sense that all the animals returned to pre-operative withdrawal threshold values after 30 min of SCS (Table 4.1).

\section{CatWalk gait analysis after partial nerve injury}

First we performed a CatWalk gait analysis after a unilateral ligation of the sciatic nerve. With respect to a reliable comparison of gait parameters between animals with nerve ligation, two aspects are of high importance: body weight and speed of gait [96,228]. First, body weight may seriously confound interpretations about CatWalk parameters such as the Mean intensity parameter of gait. Body weight decreased after the operation to $21.81 \pm 0.17 \mathrm{~g}$ compared to baseline $(22.47 \pm$ $0.16 \mathrm{~g})(95 \% \mathrm{Cl}, 0.66(0.34,0.97))$, but returned to pre-operation values within 5 days $(22.23 \pm 0.15 \mathrm{~g})(95 \% \mathrm{Cl}, 0.23(0.09,0.55))$. Second, the speed of gait may have a strong influence on gait parameters including Print area, Stance duration and Swing duration. In the present experiment no correction for speed was needed because the CatWalk crossing time did not show significant differences in the ligated animals over time (baseline $1.46 \pm 0.04 \mathrm{~s}$, DPO2 $1.46 \pm 0.25 \mathrm{~s}$, DPO7 $1.44 \pm 0.35 \mathrm{~s}$, DPO14 $1.37 \pm 0.26 \mathrm{~s}$, pre-SCS $1.38 \pm 0.06 \mathrm{~s}, \mathrm{SCS} 301.43 \pm 0.25 \mathrm{~s}$ and SCS90 $1.43 \pm 0.20 \mathrm{~s})$ (ANOVA $p>0.05$ ). 

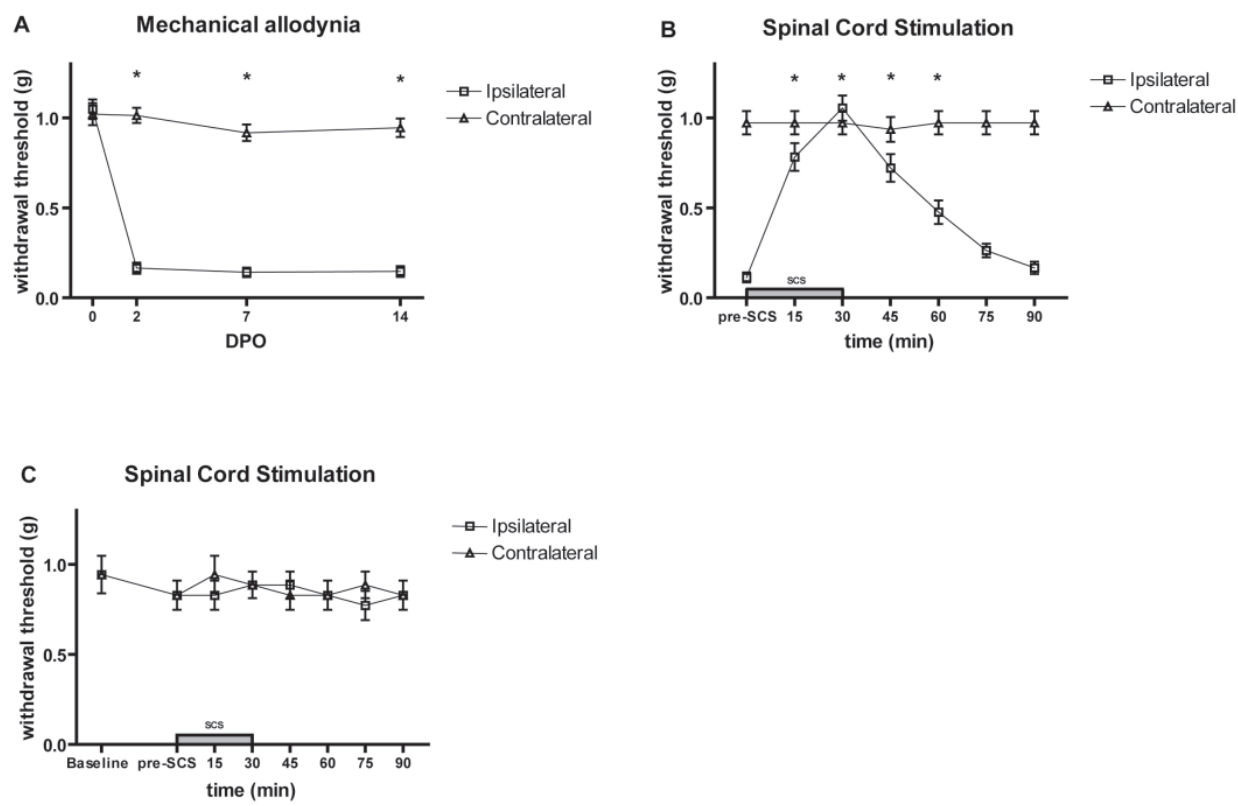

Figure 4.1. Development of mechanical allodynia after partial ligation of the sciatic nerve. All data are represented as mean \pm SEM. (A) Decrease in withdrawal threshold of the ipsilateral hind paw to mechanical (von Frey) stimulation following the Seltzer ligation $(N=29)$. No change in withdrawal threshold of the contralateral paw could be detected. (B) Spinal Cord Stimulation $(N=22)$ resulted in a rapid increase of the mean withdrawal threshold of the ipsilateral hind paw. After $15 \mathrm{~min}$ of stimulation withdrawal threshold reached $0.78 \pm 0.08$ g. Maximum stimulation effect to preneuropathy level was achieved after $30 \mathrm{~min}$, which was statistically different from pre-SCS values $(p<$ $0.001)$. Statistical significance $(p<0.05)$ compared to that at pre-SCS is indicated with asterisks. After cessation of SCS, withdrawal threshold gradually decreased to $0.26 \pm 0.04 \mathrm{~g}$ at $75 \mathrm{~min}$ and was not significantly different compared to pre-SCS $(p>0.05)$. SCS did not affect the withdrawal threshold of the contralateral paw. (C) Effect of SCS on the withdrawal threshold in non-ligated animals $(N=7)$. No effect of SCS on the withdrawal threshold of both the ipsilateral and contralateral paw could be detected.

Partial ligation of the sciatic nerve did result in a significantly changed Intensity of the ipsilateral paw print. A decrease to $74.92 \pm 6.64 \%$ at DPO2 (compared to baseline) $(p<0.001)$ was observed (Figure 4.2A). Despite the fact that from DPO2 a gradual increase was noted, still a small but significant difference could be observed between the Intensity at baseline and DPO14 (84.31 $\pm 5.71 \%, p<$ 0.05 ). No changes in Intensity of the contralateral paw were observed (Figure $4.2 \mathrm{~B})$.

The Stance duration of the left hind paw in the Seltzer group significantly decreased to $55.14 \pm 6.87 \%$ at DPO2 compared to baseline $(p<0.001)$. An increase in the Stance duration was noted over time and at DPO14 (80.64 \pm 6.08 $\%$ no significant difference could be observed compared to baseline (Figure 
4.2A). No changes in the Stance duration of the contralateral paw could be detected (Figure 4.2B).

A significant increase of the Swing duration of the ipsilateral paw was observed at DPO2 $(116.39 \pm 11.39 \%, p<0.001)$ and DPO7 $(120.90 \pm 12.89 \%, p<0.01)$ compared to baseline. At DPO 14, the Swing duration (103.07 $\pm 8.45 \%$ ) returned to baseline values (Figure 4.2A). No changes in the Swing duration of the contralateral paw were observed (Figure 4.2B).

\section{CatWalk gait analysis after Spinal Cord Stimulation}

To study the effect of the implantation procedure, the CatWalk parameters of preSCS and DPO14 were compared in the ligated animals. No effect could be detected for the Intensity and Stance duration. However an increase in Swing duration of the ipsilateral paw was noted $(103.07 \pm 8.45 \%$ at DPO14 compared to $131.46 \pm 4.37 \%$ at pre-SCS, $p=0.007)$. The implantation did not affect the CatWalk parameters of the contralateral paw.

For CatWalk analysis after SCS, 19 animals were included. Three out of the 22 animals did not cross the CatWalk appropriately. CatWalk crossing times of these remaining 19 mice at pre-SCS $(1.38 \pm 0.06 \mathrm{~s})$ did not differ from the crossing times before implantation $(1.37 \pm 0.26 \mathrm{~s}, \mathrm{p}>0.05)$.

No effect on all CatWalk parameters studied could be observed after 30 min of SCS, for both the ipsilateral and contralateral hind paw. Intensity, Stance duration and Swing duration showed no changes compared to those at pre-SCS or $60 \mathrm{~min}$ after cessation of SCS (Figure 4.2C and D).

In the control animals no changes in weight could be observed after the implantation compared to baseline $(20.57 \pm 0.20 \mathrm{~g}$ and $20.80 \pm 0.21 \mathrm{~g}$ respectively $(95 \% \mathrm{Cl}, 0.23,(-0.148,0.60))$. Also the crossing times did not differ over time (baseline $1.63 \pm 0.08 \mathrm{~s}$, pre-SCS $1.48 \pm 0.10 \mathrm{~s}$, SCS30 $1.45 \pm 0.05 \mathrm{~s}$ and SCS90 $1.36 \pm 0.05 s$ (ANOVA $p>0,05)$ and were not significantly different compared to the Seltzer animals.

Thirty minutes of SCS did not result in any changes in CatWalk parameters $(N=7)$ in either the ipsilateral or contralateral hind paw (Figure 4.2E and F). Even sixty minutes after the termination of SCS no changes in CatWalk parameters could be observed (Figure 4.2E and F). 
A

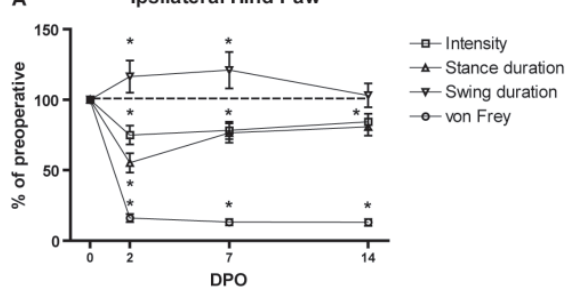

C
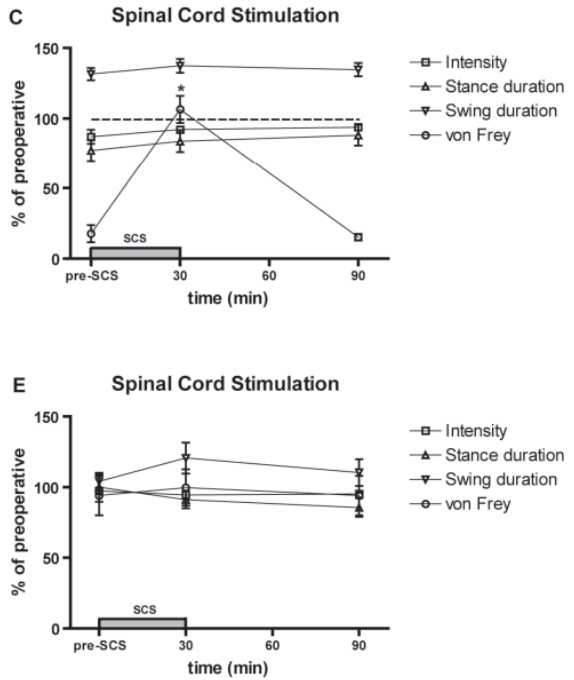
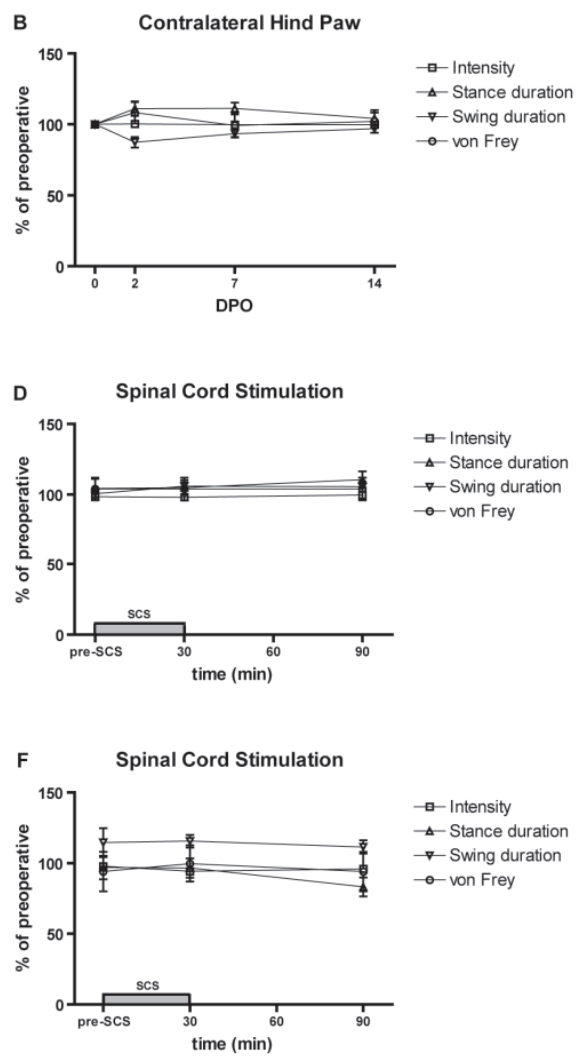

Figure 4.2. Effect of partial ligation of the left sciatic nerve and SCS on gait using the CatWalk. All data are represented as percent of pre-operative (mean \pm SEM). (A) Decrease of the Intensity to $74.92 \pm$ $6.64 \%$ at DPO2 compared to baseline. After DPO2, the Intensity started to increase to $84.31 \pm 5.71$ $\%$ of baseline at DPO14, but was still different from baseline. Reduction of the Stance duration to $55.14 \pm 6.78 \%$ at DPO2 compared to baseline. Over time the Stance duration returned to baseline and at DPO14 no significant difference could be observed compared to baseline. A statistically significant increase of the Swing duration could be observed at DPO2 and DPO 7, but returned to baseline levels at DPO14. Withdrawal threshold of the ipsilateral paw decreased to $16.02 \pm 2.88 \%$ of baseline values and remained unchanged at DPO14 (13.04 $\pm 2.43 \%$ ). (B) No effect on Intensity, Stance duration, Swing duration or on responses to von Frey filaments was observed in the contralateral paw. (C) No effect of SCS on Intensity, Stance duration and Swing duration was observed. Thirty minutes of SCS completely returned withdrawal threshold to pre-neuropathy levels. Antiallodynic effect of SCS lasted until t $=75$ min. (D) No effect of SCS on the CatWalk parameters of the contralateral paw was noted. (E, F) SCS did not result in any changes in the CatWalk parameters or withdrawal threshold (von Frey) in the non-ligated control animals $(N=7)$. Statistical significance $(p<$ $0.05)$, compared to baseline is indicated with asterisks (A and B). Statistical significance $(p<0.05)$, compared to pre-SCS is indicated with asterisks (C and D). 


\section{Discussion}

The present study demonstrates the use of SCS as a treatment for mechanical allodynia in a mouse model of chronic neuropathic pain. Complete return to preneuropathy levels was observed after $30 \mathrm{~min}$ of SCS. The anti-allodynic effect remained up to $60 \mathrm{~min}$ after cessation of SCS. We also showed that the severity of mechanical allodynia 16 days after the Seltzer ligation is not related to the therapeutic response to SCS in C57BL/6 mice with chronic neuropathic pain. The CatWalk gait analysis did detect gait changes after the Seltzer ligation, but these changes became less pronounced over time. No changes in gait were detected after 30 min of SCS.

Gait analysis using the CatWalk was initially introduced to study functional deterioration and recovery after spinal cord injury $[61,66,96]$ in rats. But it was also proposed to be a novel method to assess mechanical allodynia in a model of chronic neuropathic pain [226]. Nowadays the CatWalk is also being used in acute inflammatory pain models to detect development of mechanical allodynia [58] and behavior associated with inflammatory ankle-joint pain and therapeutic interventions [3]. In a carrageenan-induced monarthritis model the effect of the analgesics Morphine and Rofecoxib on CatWalk gait parameters was examined. It was demonstrated that Morphine and Rofecoxib could partially reverse carrageenan-induced changes in CatWalk parameters [3]. On the other hand, Morphine and Ibuprofen were not able to reverse CatWalk changes in a chronic constriction injury model, whereas they were able to inhibit mechanical allodynia when the von Frey test was used [127]. Recently, the use of the CatWalk for assessment of mechanical allodynia in acute inflammatory pain was disputed. It was reported that at 2 weeks after carrageenan injection no changes in the CatWalk parameters Intensity, Stance phase and Swing phase could be detected, whereas mechanical allodynia (as measured with the von Frey test) was still present [59]. Furthermore, a discrepancy was shown between the onset of mechanical allodynia and gait changes after complete sciatic nerve resection [43]. A clear decrease in CatWalk gait parameters was observed immediately after resection of the sciatic nerve, but mechanical allodynia (measured with the von Frey test) was found to develop only after a delay of 2 weeks [43]. On the other hand, both the CatWalk parameter Print area and von Frey values were both decreased when full motor function recovery was observed in rats with sciatic nerve crush (using the De Medinacelli method) [225].

When measuring "chronic neuropathic pain" with the use of the CatWalk, one must appreciate the type of injury used for the development of the chronic pain state. Many different experimental models of peripheral mono-neuropathy have been developed in rats, including chronic constriction injury of the sciatic nerve [10], partial ligation of the sciatic nerve [187] and ligation of the L5 root of the sciatic nerve [92]. To our knowledge, the $\mathrm{CCl}$ model, sciatic nerve crush model and the carrageenan rat model have been used to assess prolonged changes in 
gait using the CatWalk $[59,225,226]$. Because gait is a complex process, involving the function of both motor and sensory axons, damage to either of these can affect gait. Degeneration and axonal loss of all axon types were reported after chronic constriction of the sciatic nerve $[8,29,227]$ and similar pathological changes were reported in the Seltzer type ligation [117]. These results suggested that both motor and sensory axons are equally lost following damage to the sciatic nerve. Therefore the behavioral changes as measured by the CatWalk resulting from damage to the sciatic nerve at mid-thigh level (involving both motor and sensory nerve fibers) are likely to be a compromise between pain and motor-related alterations.

Obviously we were not able to detect any effect of SCS on the CatWalk gait parameters. At the same time the animals did show a profound effect of SCS on the withdrawal threshold as measured with the von Frey filaments. This strongly suggests that, in this model of chronic neuropathic pain, the CatWalk gait analysis does not allow the investigation of the effect of SCS on mechanical allodynia.

An interesting observation is the fact that in C57BL/6 mice a $100 \%$ response to SCS was observed and that no relation could be detected between the severity of mechanical allodynia and the effect of SCS.

Previously, it was shown that a subdivision of Sprague-Dawley rats into 3 groups could be made based on the degree of mechanical allodynia in Sprague-Dawley rats [197]. The most severely allodynic rats (withdrawal threshold 0.16-1.0 g) did not respond to SCS whereas the mildly allodynic rats (withdrawal threshold 8-25 g) showed a return to pre-injury withdrawal threshold after 30 min of SCS. In our mouse experiment even the most severely allodynic animals, with a reduction in withdrawal threshold to $0.8 \%$ of pre-injury values, responded optimally to SCS with a complete return to pre-injury withdrawal threshold. The difference in response to SCS after a peripheral nerve injury may be related to differential heritability of nociception. Compared to other mouse strains, the C57BL/6 is relatively resistant to mechanical allodynia $[144,145]$. It should be noted that in rats also genetic differences occur in sensitivity to mechanical allodynia [42]. Nevertheless, the findings in the Sprague-Dawley rats on the relation between efficacy of SCS and degree of mechanical allodynia have been shown to be comparable to those in patients with CRPS-I [222]. An explanation for the difference in efficacy of SCS between the Sprague-Dawley rats with chronic neuropathic pain and C57BL/6 mice with chronic neuropathic pain could be of a technical nature. The effect of SCS and the way in which the dorsal column is exited is related to current density, fiber diameter and distance between the nerve fiber and the cathode [76]. The cathode used in mice $(2.25 \times 0.76 \times 0.025 \mathrm{~mm})$ is smaller than that used in rats $(3.00 \times 1.00 \times 0.025 \mathrm{~mm})$. With consistent electrode conductivity, current density is inversely proportional to the electrode size. Whereas the contact area of the stimulating electrode decreases for specific current intensity, the current density increases [76]. This increase in current density might result in the stimulation of more fibers located in the dorsal 
funiculus, eventually resulting in a better anti-allodynic effect in allodynic mice as compared to rats.

One of the proposed mechanisms underlying the effect of SCS in neuropathic pain is the facilitation of the GABAergic system [33]. Administration of a $\mathrm{GABA}_{b}$ agonist (baclofen) potentiates the behavioral response to SCS in rats previously responding poorly to SCS. Loss of GABA and its synthesizing enzyme glutamate decarboxylase (GAD) from the dorsal horn has been reported following nerve injury in rats $[22,48,78,147]$. In contrast, an initial increase in GABA was detected and no changes in GAD were observed in mice with spared nerve injury [183]. This could be another explanation for the $100 \%$ response to SCS in mice.

Furthermore, it has been recently suggested that the anti-allodynic effect of electrical stimulation could be attributed to the down-regulation of NR1 phosphorylation in a rat model of visceral hypersensitivity [211]. The phosphorylation of the NMDA receptor has been suggested as an important component in the development of central sensitization and chronic pain $[60,246,247]$. One could speculate that SCS in chronic neuropathic pain has a more pronounced effect on the phosphorylation of the NMDA receptor and, if so, is more efficient in C57BL/6 mice than in Sprague-Dawley rats.

\section{Conclusions}

We have shown that SCS in a mouse model of chronic neuropathic pain completely abolishes mechanical allodynia. Severity of mechanical allodynia is not a prediction for the efficacy of SCS in this mouse model. We have demonstrated that the CatWalk cannot be used in this model of chronic neuropathic pain to assess mechanical allodynia and the effect of SCS on mechanical allodynia.

The present SCS-mouse model opens new avenues for studies on the mechanisms underlying neuromodulation as a therapy in chronic neuropathic pain. 



\section{Part II}

Improving Spinal Cord Stimulation 



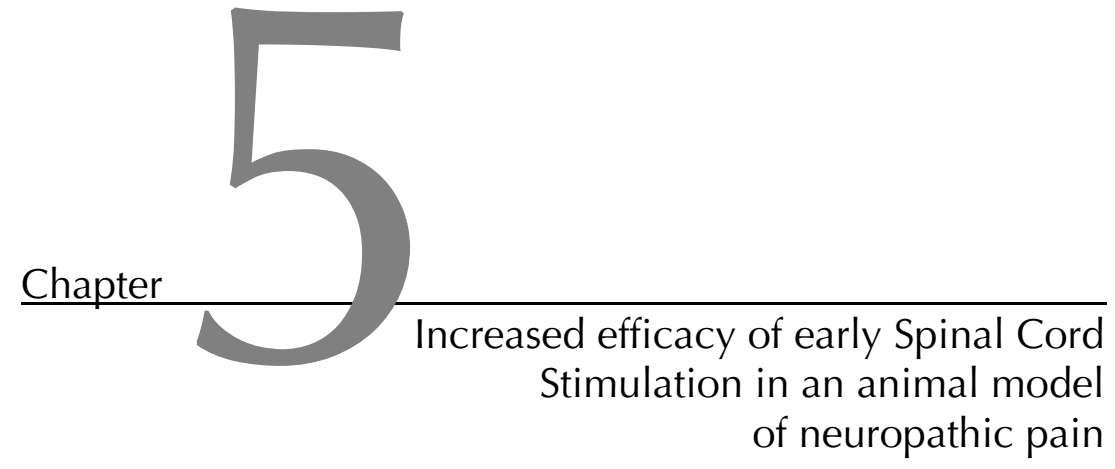

M. Truin M. van Kleef

B. Linderoth

H. Smits

S.P.M. Janssen

E.A.J. Joosten

European Journal of Pain 2010,15 (2): 111-7 


\section{Abstract}

\section{Background and Aims}

Although Spinal Cord Stimulation (SCS) is an established treatment for chronic neuropathic pain, pain relief is still not successful in a large group of patients. We suggest that the success of SCS may be related to the timing of SCS during the development of chronic neuropathic pain. We therefore compared the effect of SCS applied after $24 \mathrm{~h}$ of neuropathic pain (early SCS) and after 16 days of neuropathic pain (late SCS).

\section{Methods}

For early SCS, male Sprague-Dawley rats $(N=13)$ were implanted with an SCS device, followed by a partial ligation of the sciatic nerve. Using von Frey monofilaments, tactile allodynia was assessed 24h after ligation. Animals with tactile allodynia received $30 \mathrm{~min}$ of SCS. Withdrawal thresholds were assessed just before SCS, during SCS and until the return to pre-stimulation withdrawal threshold. Results were compared with the data from late SCS $(N=29)$.

\section{Results}

Out of the 13 allodynic animals that received early SCS, 10 (77\%) responded to SCS with significantly increased withdrawal thresholds, compared to $38 \%$ in the late SCS group. The increase of the withdrawal threshold in the early SCS group could still be noticed 90 min after termination of SCS. In more than half of these animals, pre-stimulation withdrawal thresholds were reached only the next day. Early SCS resulted in an increased number of responders to SCS and furthermore an increased duration of the effect of SCS as compared to late SCS.

\section{Conclusion}

Early SCS treatment of neuropathic rats is more effective as compared to the late SCS treatment. 


\section{Introduction}

In patients with complex regional pain syndrome type 1 (CRPS-1) the pain is reported to be debilitating, greatly influencing daily life. This pain is neuropathic in origin and is thought to be the result of both peripheral and central sensitization. Spinal cord stimulation (SCS) in a well selected group of CRPS-1 patients is considered to be an effective treatment, although approximately $50 \%$ of patients are eligible for permanent implant and satisfactory pain relief subsides over time $[87,88]$. It was therefore suggested that better patient selection and optimal timing of the procedure might improve the outcome of SCS in CRPS-1 patients [203].

Recently, it has been reported that the absence of mechanical allodynia in patients with CRPS-1 predicts a good outcome after 1 year of SCS (81\%), whereas in patients with severe allodynia, the success of SCS was much lower $(31 \%)$ [222]. This is in line with the results from an experimental animal model of chronic neuropathic pain and SCS, where animals with severe allodynia did not benefit from SCS [197]. Furthermore, it has been shown that the severity of touch evoked allodynia in patients with CRPS is positively correlated with the progression of the disease [186]. These findings suggest that the severity of allodynia might reflect the degree or severity of central sensitization and is time dependent. Consequently, severe or long lasting central sensitization predicts a poor outcome for SCS in patients with CRPS-1, where an early intervention could improve the outcome of SCS by limiting or attenuating central sensitization. Indeed, several case studies have reported an improved outcome, when SCS was used in an early stage of the disease [71,178,223].

We therefore set to determine if early SCS (1 day after induction of mononeuropathy) could improve the effect on hypersensitivity to innocuous stimuli (mechanical allodynia) compared to late SCS (16 days of neuropathic pain) in an animal model of neuropathic pain. 


\section{Methods}

\section{Animals}

Male Sprague-Dawley rats, weighing 300-350 g, were used in the experiments. The animals were individually housed under temperature controlled conditions at $21 \pm 1{ }^{\circ} \mathrm{C}$, with a normal 12:12 h light/dark cycle. The animals had free access to food pellets and water.

\section{General}

All experiments were performed in accordance with the European Directive for the Protection of Vertebrate Animals Used for Experimental and Other Scientific Purposes (86/609/EU) and were approved by the Committee for Experiments on Animals of Maastricht, The Netherlands.

Surgery was performed in anesthetized animals under aseptic conditions. Induction and maintenance of general anesthesia was performed by isoflurane inhalation. Constant body temperature was maintained by placing the animal on an automated heating pad $\left(37.5 \pm 0.5^{\circ} \mathrm{C}\right)$, (IDEE, University Maastricht). If signs of extreme weight loss (>15\% of body weight at baseline), absence of grooming or appearance of cachectic behavior were observed, the animal was euthanized.

\section{Induction of neuropathic pain}

A unilateral ligation of the left sciatic nerve was performed as described by Seltzer [187]. The left sciatic nerve was exposed by blunt dissection and carefully freed from surrounding connective tissue. Distal to the posterior biceps semitendinosus, but proximal to the little fat pad that lies a few millimeters distal to this site, the sciatic nerve was partially ligated. An $8 / 0$ non-absorbable silk suture with a reverse cutting micro needle was used to ligate approximately $1 / 3$ to $1 / 2$ of the diameter of the left sciatic nerve. After the ligation, the wound was closed with a 4/0 silk suture. In the early SCS group, the Seltzer operation was performed 2 days after the implantation of the SCS device. In the animals in the late SCS group, the Seltzer operation was performed and the development of tactile hypersensitivity (mechanical allodynia), based on paw withdrawal thresholds to innocuous mechanical stimuli was followed for 16 days as reported previously [197].

\section{Implantation of the SCS system}

In the animals receiving early SCS, the SCS system was implanted prior to the partial ligation of the sciatic nerve. The same implantation procedure and SCS system were used as reported earlier [197]. In brief, a rectangular platinum iridium cathode (3.00 x 1.00 × 0.10 mm, Medtronic Bakken Research, Maastricht) (Fig. 1) was implanted epidurally by a small laminectomy at the T13 level. The wire of the cathode was secured to the spinosus process of T12 to prevent movement of the lead. The anode (diameter of $6.0 \mathrm{~mm}$ and thickness of $0.10 \mathrm{~mm}$ ) 
(Figure 5.1) was placed subcutaneously. Both leads were tunneled subcutaneously to the neck of the animal and connected to micro plugs, which were sutured to the skin of the neck of the animal. Animals were allowed to recover for 2 days after electrodes implantation. In the late SCS group, the SCS device was implanted after 14 days of neuropathy according to the same procedure.

For SCS, a Grass S88 stimulator (Astro-med Grass, Warwick, USA) fitted with a Grass SIU-5 stimulus isolator and a Grass constant current unit was used. The stimulus paradigm consisted of the assessment of the motor threshold (frequency $2 \mathrm{~Hz}$, pulse width $0.2 \mathrm{~ms}$ ) followed by $30 \mathrm{~min}$ of SCS (2/3 of motor threshold, frequency $50 \mathrm{~Hz}$, pulse width $0.2 \mathrm{~ms}$ ) as reported earlier [33,36,180,197].

To study the effect of SCS in control animals, electrodes were implanted in nonneuropathic unligated rats. These animals were also allowed to recuperate after surgery for $48 \mathrm{~h}$, before being submitted to $30 \mathrm{~min}$ of SCS.

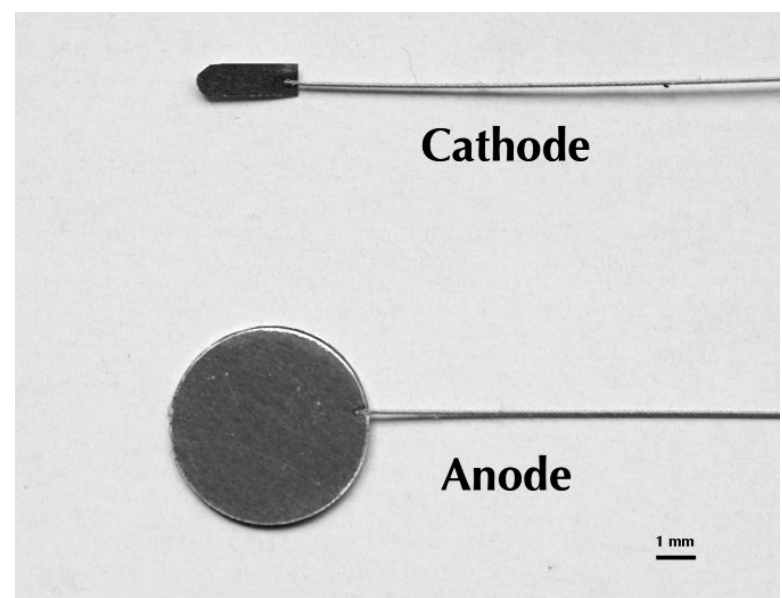

Figure 5.1. Electrodes used for SCS. The cathode $(3.00 \times 1.00 \times 0.10 \mathrm{~mm})$ was placed on the dura of the spinal cord and the anode (diameter $6.0 \mathrm{~mm}$ and thickness $0.10 \mathrm{~mm}$ ) was placed subcutaneously.

\section{Behavioral testing}

All animals were tested for their behavioral response to innocuous mechanical stimuli, using von Frey monofilaments. Testing took place in a set of Plexiglas cages with an elevated wire mesh floor. Prior to testing, the animals were allowed to adapt for $30 \mathrm{~min}$. After acclimatization, the von Frey filaments were applied, in an order of increasing stiffness, through the wire mesh floor to the mid-plantar surface of the left or right hind paw until the filaments bent slightly. A positive response was scored when the animal showed a brisk withdrawal of the hind paw to 3 of the 5 stimuli presented. The animals were tested using 14 von Frey monofilaments (Stoelting CO, IL, USA) with logarithmically incremental stiffness (0.16, 0.4, 0.6, 1.0, 1.4, 2.0, 4.0, 6.0, 8.0, 10.0, 15.0, 26.0, 60.0, and $100 \mathrm{~g})$, corresponding to the linear perceived intensity $(3.22,3.61,3.84,4.08,4.17,4.31$, 
4.56, 4.74, 4.93, 5.07, 5.18, 5.46, 5.88 and 6.10). These values are achieved according to the formula: $\log 10$ (mg x 10) (Stoelting). Animals which did not display a decrease of the withdrawal threshold of $\geq 2$ von Frey filaments after the Seltzer ligation as compared to pre-nerve injury withdrawal thresholds were excluded and defined as "non-allodynic".

Prior to early stimulation (pre-SCS) withdrawal thresholds were measured. Rats were tested 15 and 30 min following initiation of SCS. After 30 min SCS was terminated and the animals were tested the following 30, 60, 90, 120, $150 \mathrm{~min}$. Rats who did not return to pre-SCS withdrawal thresholds after $150 \mathrm{~min}$ were tested once more the next day. Animals receiving late SCS were tested at the same time points as rats receiving early SCS, except testing was stopped 60 min after termination of SCS. A responder to SCS was defined when SCS resulted in an maximum possible effect (MPE) > 50\%. MPE was calculated for each individual animal according to the following formula: MPE \% = (withdrawal threshold after $30 \mathrm{~min}$ of SCS - withdrawal threshold pre-SCS)/(pre-nerve injury withdrawal threshold - withdrawal threshold pre-SCS) x $100 \%$. The withdrawal thresholds of the unligated control animals were tested before implantation of the electrodes. Two days after the surgery, withdrawal thresholds were again assessed according to the same paradigm as the late SCS group.

\section{Experimental procedures}

Two SCS paradigms were compared: "early" SCS was compared with "late" SCS (data from late SCS were acquired previously in our lab) [197], where early SCS was defined as SCS applied $24 \mathrm{~h}$ after the appearance of tactile hypersensitivity and late SCS was defined as SCS applied after 16 days of hypersensitivity (Fig.2).

Due to a recuperation period of 2 days after the implantation of the electrodes, we implanted the SCS device in the early SCS group before nerve injury. Two days after the electrode implantation, the sciatic nerve was partially ligated as described by Seltzer [187]. In the late SCS group, the SCS device was implanted 14 days after the partial ligation of the sciatic nerve and the animals were allowed to recuperate for 2 days. Both groups received $30 \mathrm{~min}$ of SCS. After each experiment, the animals were euthanized and the position of the electrode (cathode) was verified. This was done using the spinosus of the 10th and 11th vertebrae (which are easily identified) and the $13^{\text {th }}$ rib as anatomical reference points. 


\section{Statistical analysis}

The tactile withdrawal thresholds to von Frey filaments are presented as mean \pm standard error of the mean (SEM). For statistical analysis of differences in the withdrawal thresholds over time, the non-parametric Friedman test, followed by Dunn's post hoc test, was used. For comparison between the different groups, the Mann-Whitney test was used. For analysis of the area under the curve (AUC), a unpaired $t$-test or one-way ANOVA compared differences between groups. $p<$ 0.05 was considered to be statistically significant in all tests.

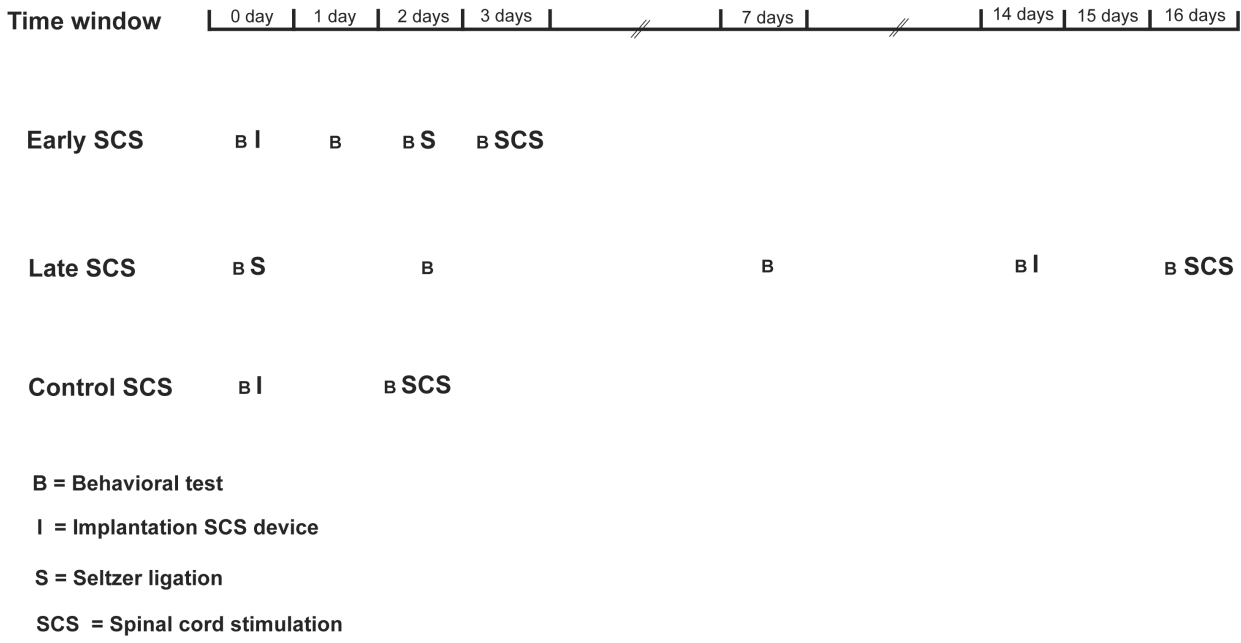

Figure 5.2. Experimental procedures: timeframe for early spinal cord stimulation, late spinal cord stimulation and stimulation of uninjured animals.

\section{Results}

\section{General observations}

In the early SCS group, out of a total of 16 animals that received a unilateral partial ligation of the sciatic nerve, three animals did not display tactile hypersensitivity after the Seltzer procedure and were excluded from the study. The remaining 13 animals qualified as hypersensitive (allodynic) and received SCS. In the late SCS group, 29 of the 32 animals showed a decrease of the withdrawal threshold of $\geq 2$ von Frey filaments after the Seltzer ligation and thus qualified as hypersensitive (allodynic). These 29 animals all underwent late SCS. 
A Development of mechanical allodynia

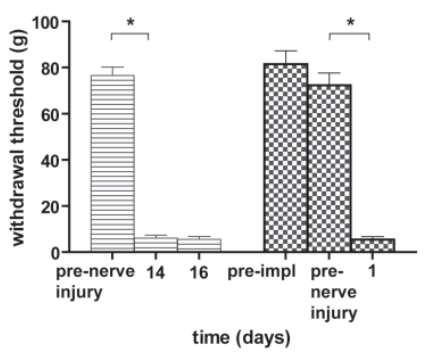

C Spinal Cord Stimulation

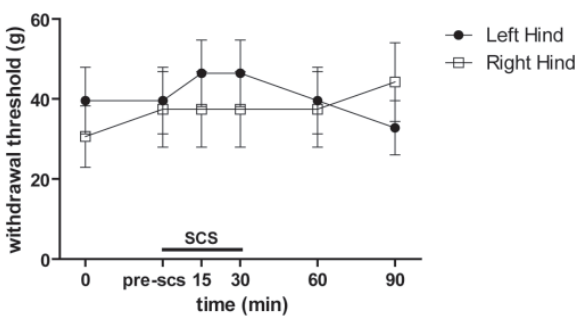

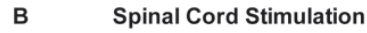

$\boxminus$ Late SCS Borly SCS

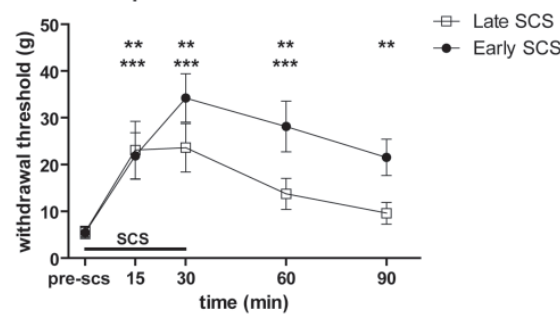

Figure 5.3. Development of tactile hypersensitivity (mechanical allodynia) after Seltzer ligation and the effect of SCS in the hypersensitive total population and uninjured animals. All data are represented as mean \pm SEM or maximal possible effect (MPE). (A) Decrease in withdrawal threshold of the ipsilateral hind paw to mechanical (von Frey) stimulation following Seltzer ligation. For the late SCS group $(N=$ 29) implantation did not affect the withdrawal threshold (14 and 16 days). The same is true for the early SCS group ( $N=13$ ) (pre-implantation and pre-nerve injury). (B) Spinal cord stimulation. In both groups, SCS resulted in an increase of the withdrawal threshold of the ipsilateral hind paw. Maximal increase of the withdrawal threshold was reached after $30 \mathrm{~min}$ of SCS. After termination of SCS, withdrawal thresholds gradually decreased in both groups over time. In the early SCS group $(N=13)$, the effect of SCS was still present at $t=90 \mathrm{~min}$, whereas in the late SCS group $(N=29)$ withdrawal thresholds were back at pre-SCS withdrawal thresholds at $t=90$ minutes. (C) Effect spinal cord stimulation in uninjured rats. Both the implantation alone and $30 \mathrm{~min}$ of SCS did not affect the withdrawal thresholds of the left and right hind paw. * Statistically significant difference with $p<0.05$ compared to pre-nerve injury. ${ }^{* *}$ Statistically significant difference with $p<0.05$ compared to pre-SCS (early group). ${ }^{* * *}$ Statistically significant difference with $p<0.05$ compared to pre-SCS (late group).

\section{Development of tactile hypersensitivity}

Pre-nerve injury withdrawal thresholds of the ipsilateral hind paws in the early $(81.5 \pm 5.8 \mathrm{~g})(N=13)$ and late SCS group $(76.6 \pm 3.7 \mathrm{~g})(N=29)$ were comparable (Figure 5.3A). One day after the partial ligation of the sciatic nerve the withdrawal threshold of the ipsilateral paw in the early SCS group decreased from $81.5 \pm 5.8 \mathrm{~g}$ to $5.5 \pm 1.3$ ( $t 1=1$ day after nerve ligation) $(p<0.05)$, and a similar decrease in the withdrawal threshold was noted in the late SCS group 14 
days after the Seltzer ligation $(76.6 \pm 3.7 \mathrm{~g}$ to $5.6 \pm 1.3 \mathrm{~g})(p<0.05)$ (Figure 5.3A). The implantation of the SCS device did not affect the withdrawal thresholds of the ipsilateral hind paws in both groups $(p>0.05)$ (Figure 5.3A). No effect of the ipsilateral ligation could be detected in the withdrawal threshold of the contralateral paw.

\section{Spinal cord stimulation in the total population}

In the total population of animals with tactile hypersensitivity (mechanical allodynia) in the late SCS group $(N=29)$ and early SCS group $(N=13) 30$ min of SCS resulted in a gradual increase of the withdrawal threshold of the ipsilateral paw (Figure 5.3B). Pre-SCS withdrawal thresholds did not differ between both groups and the maximal increase in withdrawal threshold was observed at $30 \mathrm{~min}$ of SCS (SCS30). Early SCS resulted in a significant increase from $5.5 \pm 1.3 \mathrm{~g}$ to $34.2 \pm 5.2 \mathrm{~g}$ after $30 \mathrm{~min}$ of early SCS compared to pre-SCS withdrawal thresholds $(p<0.0001)$ (Figure 5.3B). A comparable increase from $5.6 \pm 1.3 \mathrm{~g}$ to $23.6 \pm 5.2$ $\mathrm{g}(p<0.0001)$ was observed after $30 \mathrm{~min}$ of late SCS (Figure 5.3B). After termination of SCS there was a gradual return to pre-SCS withdrawal thresholds in both groups. In the early SCS group however, withdrawal thresholds did not reach pre-SCS withdrawal thresholds at $t=90 \mathrm{~min}(21.5 \pm 3.9 \mathrm{~g})$ (60 min after cessation of SCS), where animals receiving late SCS returned to their pre-SCS withdrawal thresholds at $t=90$ minutes $(9.6 \pm 2.3 \mathrm{~g})$. No effect of the $30 \mathrm{~min}$ of SCS could be detected in the withdrawal threshold of the contralateral paw in both groups.

Implantation of the electrodes in the control group $(N=5)$ did not affect the withdrawal thresholds of the left hind paw or right hind paw (Figure 5.2C). During the 30 min of SCS and after termination of SCS, withdrawal thresholds did not change as compared to pre-SCS withdrawal thresholds (Figure 5.3C).

When the effect of SCS was expressed in \% MPE for each time point, 30 min of early SCS resulted in a $66.8 \pm 5.0 \%$ MPE. This was significantly different compared to the MPE at $30 \mathrm{~min}$ of SCS in the late SCS group $(36.0 \pm 9.6 \%)(p=$ 0.04). After termination of SCS, the MPE of the early SCS group remained significantly increased as compared to the late SCS animals (Figure 5.4A). The AUC of the MPE of the early SCS and late SCS show a significant difference between the groups ( $p=0.008)$, were the AUC of the early SCS group is significantly greater than the AUC of the late SCS group (Figure 5.4B). 

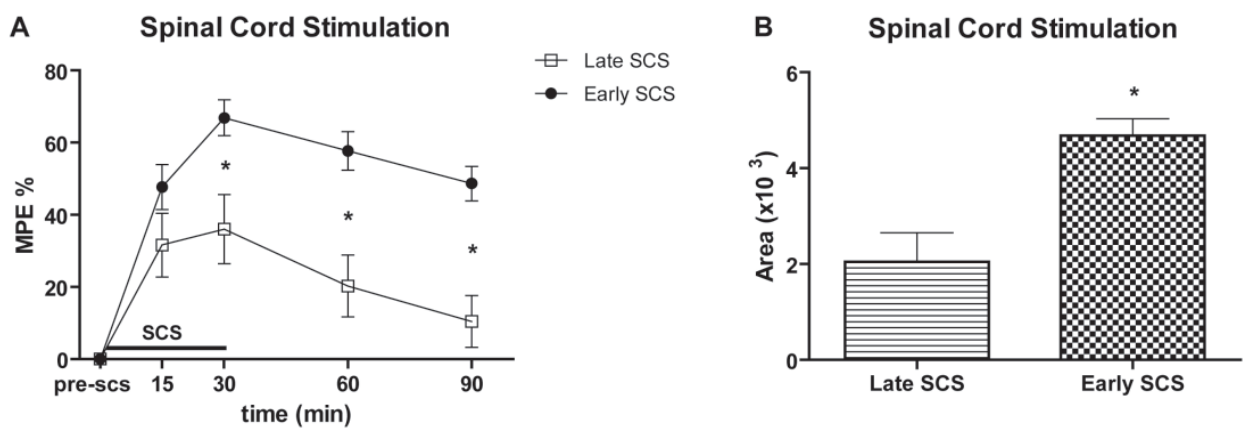

Figure 5.4. Effect of spinal cord stimulation expressed as MPE \%. (A) Early SCS resulted in a significant larger MPE after 30 min of SCS compared to late SCS. After cessation of SCS, the MPE of the early SCS group remained significantly increased over time compared to late SCS. (B) The AUC of the early SCS group was significantly larger compared to the AUC of the late SCS group. * Statistically significant difference with $p<0.05$ between late and early SCS.

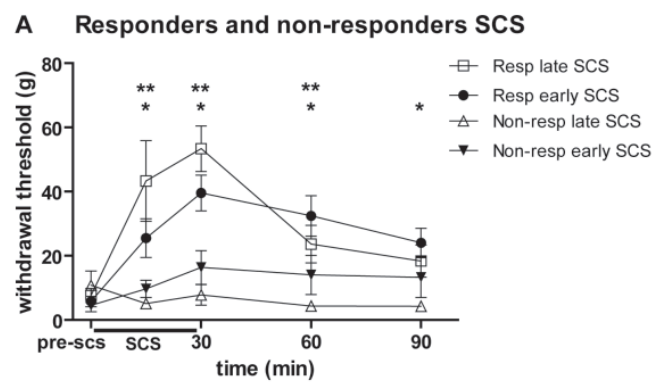

B Responders and non-responders SCS

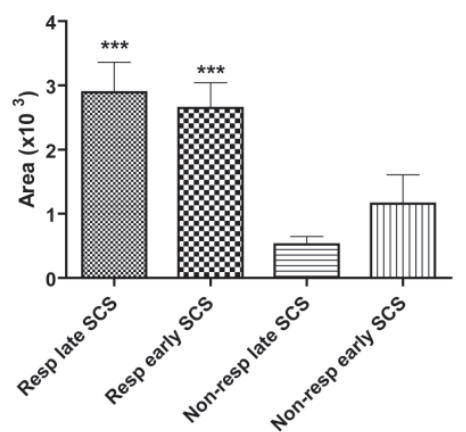

Figure 5.5. Responders and non-responders to early and late SCS based on MPE $>50 \%$ at SCS30. All data are represented as mean \pm SEM. (A) Animals responding to late SCS $(N=11)$ reached maximal withdrawal thresholds after $30 \mathrm{~min}$ of SCS. After termination of SCS, the withdrawal thresholds decreased and reached pre-SCS withdrawal thresholds at $t=90$ minutes. Early SCS $(N=10)$ resulted in a similar increase of the withdrawal thresholds, reaching maximum values at SCS30. After cessation of SCS, withdrawal thresholds were still increased at $t=90 \mathrm{~min}$ as compared to pre-SCS values. The nonresponders to early SCS $(N=3)$ and late SCS $(n=18)$ did not display an increase in the withdrawal threshold as a result of SCS. (B) The AUC of the responders to late and early SCS were not statistically different, but compared to the non-responders the AUC of both responders to late and early SCS were significantly increased compared to the non-responders to SCS. ${ }^{*}$ Statistically significant difference with $p<0.05$ compared to pre-SCS (early group). ${ }^{* *}$ Statistically significant difference with $p<0.05$ compared to pre-SCS (late group). ${ }^{* * *}$ Statistically significant difference with $p<0.05$ between responders and non-responders to SCS. 


\section{Responders and non-responders to SCS}

Next, we calculated the MPE at SCS30 for each individual animal in both groups, using our definition of a responder to SCS (MPE > $50 \%$ ). In the early SCS group, 10 out of the 13 animals ( $77 \%$ ) could be defined as responders to SCS (MPE 73.2 $\pm 7.0 \%$ ). Late SCS resulted in $38 \%(11 / 29)$ responders to SCS (MPE $=88.3 \pm 6.2$ $\%)$. Focusing on the responders to SCS in both groups, the MPE of late SCS and early SCS revealed no statistical significant difference. Both early $(N=10)$ and late $(N=11)$ SCS resulted in a gradual increase of the ipsilateral withdrawal thresholds. Furthermore, no difference could be noted between the maximal increase in withdrawal thresholds after $30 \mathrm{~min}$ of SCS (late SCS; $53.4 \pm 7.1 \mathrm{~g}$ ) and early SCS (39.6 $\pm 5.6 \mathrm{~g})(p=0.14)$ (Figure 5.5A). After termination of SCS, a gradual decrease of the withdrawal threshold of the ipsilateral paw was noted in the responders to early SCS. The increased withdrawal thresholds $(24.0 \pm 4.6 \mathrm{~g})$ in the early SCS animals was still present at $t=90 \mathrm{~min}(60 \mathrm{~min}$ after cessation of SCS) and was significantly increased compared to pre-SCS withdrawal thresholds $(5.7 \pm 1.6 \mathrm{~g})$. In animals receiving late SCS, the withdrawal thresholds at $t=90$ min had reached pre-SCS values $(18.4 \pm 5.0 \mathrm{~g}$ and $7.6 \pm 2.2 \mathrm{~g}$, respectively, $p>$ 0.05). The animals who where defined as non-responders to SCS did not show a significant increase of the withdrawal threshold over time (Figure 5.5A). When comparing the AUC of the different groups, no difference could be observed between the responders to late SCS and early SCS or the non-responders to late SCS and early SCS $(p>0.05)$. The AUC of the responders to late and early SCS were significantly larger compared to the AUC of the non-responders to late and early SCS (Figure 5.5B).

In three animals, $90 \mathrm{~min}$ after termination of early SCS $(t=120 \mathrm{~min}$ ) pre-SCS withdrawal thresholds were reached and in seven animals the withdrawal threshold was still increased at $t=180$ minutes $\left(2 \frac{1}{2} \mathrm{~h}\right.$ after cessation of early SCS). These animals returned to pre-SCS withdrawal thresholds the next day (Figure 5.6A). Although a difference in the duration of the effect of early SCS was observed, no difference in maximal increase of the withdrawal threshold at SCS30 was noted (Figure 5.6A). The AUC of the animals only returning to pre-SCS withdrawal thresholds the next day was significantly larger than the AUC of the animals returning to pre-SCS withdrawal thresholds 90 min after cessation of SCS (Figure 5.6B). 
A Early Spinal Cord Stimulation

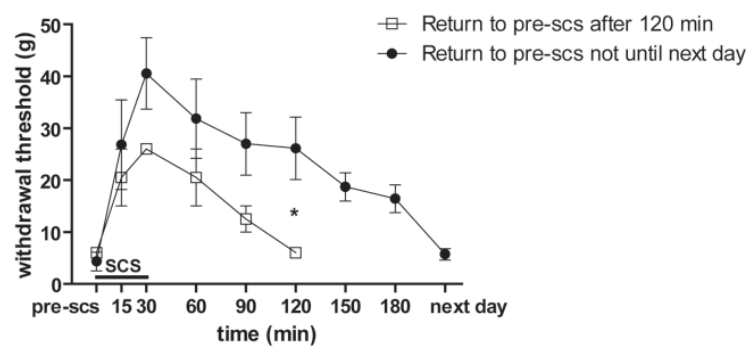

B

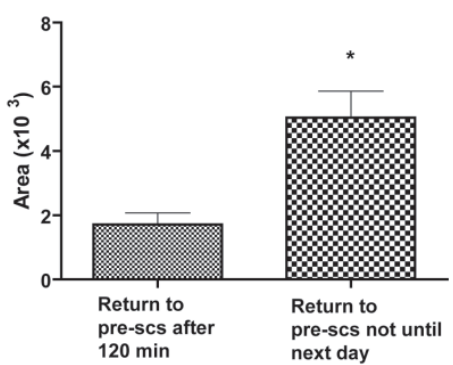

Figure 5.6. Effect of early SCS. (A) In the early SCS responders $(N=10)$, seven animals did not return to their pre-SCS withdrawal thresholds until the next day. The other three animals reached pre-SCS withdrawal thresholds at $t=120$ minutes (90 min after cessation of SCS). Only at $t=120 \mathrm{~min}$ a significant difference could be observed. (B) AUC of the animals reaching pre-SCS withdrawal thresholds the next day was significantly different compared to the AUC of the animals reaching preSCS withdrawal thresholds at 120 min. * Statistically significant difference with $p<0.05$ between preSCS $120 \mathrm{~min}$ and pre-SCS the next day.

\section{Discussion}

In the present study it was demonstrated that early SCS in animals with neuropathic pain resulted in more responders (77\%) as compared to late SCS (38 $\%)$. Furthermore, the duration of the effect of SCS was increased in the early SCS group as compared to the late SCS group. The increased duration of the effect of early SCS was not related however to the maximal increase of the withdrawal threshold.

In patients with CRPS-1, SCS is considered successful if test stimulation results in a pain reduction of more than $50 \%$ and these patients become eligible for permanent implantation [85]. After 1 year, these patients have a favorable outcome in significant pain reduction $[87,222]$. However, patients who do not respond to trial SCS neither benefit from the long term SCS. Increasing the number of responders to test stimulation is therefore much desired. We feel that in the animal model of neuropathic pain and SCS the effect of SCS reflects the effect in patients after trial stimulation, since the SCS period is relatively short. In the animal model of neuropathic pain and SCS there is no widely accepted definition for a responder to SCS and different definitions are being used $[36,121,180,197,216]$. We used the MPE, which is an established method to test the efficacy of drugs, to express the efficacy of SCS. When the definitions from the literature were used $[36,121,180,197]$, early SCS still resulted in more responders than late SCS. The MPE of $50 \%$ was chosen because this reflects a $50 \%$ reduction in mechanical allodynia, which in turn has similarities with the definition used in patients with CRPS-1 (50\% pain reduction) to define 
responders to SCS. It should be noted that the definition in patients mostly reflects a decrease in spontaneous pain and not mechanical allodynia. Interestingly however, trial stimulation in patients with CRPS-1 resulted in a marked decrease in allodynia as well as a decrease in deep burning pain [70]. To increase the number of responders to SCS by means of timing, selection or adjuvant pharmacotherapy, it is imperative to discriminate between responders and nonresponders in animal models of neuropathic pain. This to elucidate possible mechanisms responsible for the response or lack of response to SCS and thereby develop better strategies for managing pain in patients with CRPS-1.

Early SCS results in an increased number of responders to SCS and an increased duration of the anti-allodynic effect compared to late SCS, suggesting that timing of the intervention in the process of the disease is important for the success of SCS. In neuropathic pain models the central sensitization develops within hours to days and is often initiated by an increased glutamate release in the dorsal horn [81]. Another event responsible for central sensitization is the decreased inhibition of the GABA-ergic system and is suggested to be time dependent $[48,78]$. It was shown that after 3 days of neuropathic pain, GABA immunoreactivity was decreased, but still noticeable. At 2 weeks, no GABA immunoreactive profiles could be detected. Therefore, the timing of the intervention in the process of central sensitization may be crucial for the beneficial effect, not only for SCS. For instance, the implantation of cells bioengineered to synthesize GABA in the lumbar subarachnoid space in rats, with a chronic constriction injury $(\mathrm{CCl})$ [10] showed a time dependent success of increasing the withdrawal threshold of the ipsilateral paw [207]. Furthermore, pharmacological experiments on the intrathecal administration of a single dose of $\mathrm{GABA}$ in rats with $\mathrm{CCl}$ also showed a time-dependent effect [47]. Withdrawal thresholds of the ipsilateral paw significantly increased when GABA was given shortly after $\mathrm{CCI}$ (1-2 weeks), whereas late application demonstrated no effect on the withdrawal threshold.

With respect to our recent findings, this could be one explanation on how early SCS results in a higher number of responders and a longer effect as compared to late SCS. In a previous paper by Smits [196] an increase in c-Fos in the dorsal horn was reported after SCS, but it was unclear whether the c-Fos positive cells where inhibitory or excitatory cells. However, the role of inhibitory GABA-ergic cells, based on pharmalogical and biological evidence, has been documented in the literature. $[36,206,231]$. Although the behavioral signs of neuropathic pain are comparable between early and late neuropathic pain, the central sensitization and its underlying mechanisms responsible for the behavioral signs such as mechanical allodynia could be different, or in a different phase [20]. Therefore, it could be speculated that early SCS is able to increase GABA levels in almost all animals, thereby reducing the mechanical allodynia by more than $50 \%$. This effect is only temporary as cessation of SCS results in a return to the neuropathic 
state. This suggests that the effect of 30 min of early SCS is unable to stop or invert the process of central sensitization.

Our experimental data clearly demonstrate an increased efficacy of early SCS versus late SCS in a model of neuropathic pain. In this context it is interesting to note that in an experimental model of inflammatory pain, SCS worsens hyperalgesia if applied early ( $3 \mathrm{~h}$ ) after the injection of carrageenan but relieves hyperalgesia if applied later (3 days) [34]. Electrical stimulation of the rostral ventromedial medulla (RVM) was not as effective in inhibiting paw withdrawal latencies early $(3 \mathrm{~h})$ after inflammation of the hind paw as when stimulation was applied $24 \mathrm{~h}$ after inflammation [209]. Although the time points studied differ from those used in our study, these findings are remarkable. It is known that the mechanisms underlying inflammatory pain and neuropathic pain are far from identical. How SCS acts in treatment of inflammatory pain as compared to neuropathic pain is an intriguing question.

Recent work by van Eijs (paper submitted for publication) and colleagues showed no increase in response to the early test stimulations in patients with CRPS-1 (mean disease duration of 7.5 months). In $50 \%$ of the patients, test stimulation resulted in a $50 \%$ decrease of the pain score, which is comparable to the response to test stimulation in chronic CRPS-1 patients [85]. However, in another study it was shown that with a mean disease duration of 10 months, all patients with CRPS responded to the test stimulation and still had good pain reduction after 6 months of SCS [223].

An explanation on the lack of improvement of early SCS in patients (van Eijs et.al submitted) could be, that with the disease duration of 7.5 months central sensitization is robust, and therefore can no longer be affected by SCS. Earlier reports show that most of the signs and symptoms in CRPS patients are already well developed after 1 year, showing only moderate increases after 1 year [186]. Because the process of central sensitization develops within hours to days, this timeframe should be indicative for the selection of a point of intervention in order to achieve optimal results.

Our data suggest that therapies, probably including SCS, should preferentially be initiated immediately following nerve injury. This to prevent the onset or reduce severity of chronic neuropathic pain. Interestingly, it has been reported that rapid intervention by means of continuous peripheral nerve block is possible. The continuous peripheral nerve block was applied within $1 \mathrm{~h}$ after nerve injury resulting in excellent pain relieve and apparent long term efficacy [19].

In conclusion, early SCS increases the number of responders in an animal model of neuropathic pain and the anti-allodynic effect is prolonged. Although early SCS is not capable to reverse the process of central sensitization, it is indeed possible to optimize the effect of SCS early in an early stage of the disease. 


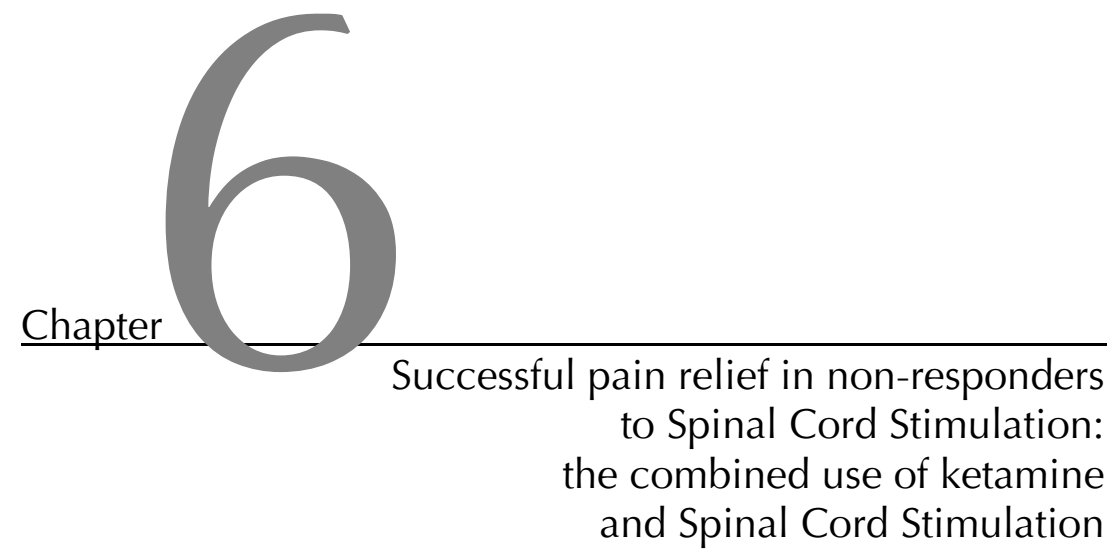

M. Truin

S.P.M Janssen

M. van Kleef

E.A.J. Joosten 


\section{Abstract}

\section{Background}

Although Spinal Cord Stimulation (SCS) is an established therapy for chronic neuropathic pain, still $30 \%$ of patients do not respond adequately to trial stimulation. These so called "non-responders" do not receive a permanent implantation for pain relief.

The induction and maintenance of central sensitization plays a pivotal role in (chronic) neuropathic pain and is thought to be the resultant of the activation of the N-Methyl-D-Aspartate (NMDA) receptor in the dorsal horn. Blocking the NMDA receptor through the use of the non-competitive blocker ketamine has shown to attenuate neuropathic pain, although the undesirable side effects limit its use.

\section{Methods}

The present study was performed to examine whether the combination of SCS with an individually determined sub-effective dose of intrathecal (i.t.) ketamine could convert non-responders into responders in rats with chronic neuropathic pain. Rats received a partial ligation of the sciatic nerve for the induction of neuropathic pain. Animals with tactile hypersensitivity to von Frey monofilaments ( $n=15$ ) received 30 minutes of SCS. Non-responders to SCS $(n=8)$ received their individually determined sub-effective i.t. dose of ketamine followed by $30 \mathrm{~min}$ of SCS. Also the responders to SCS $(n=7)$ received the individually determined subeffective i.t dose of ketamine followed by 30 min of SCS.

\section{Results}

In the 8 non-responders, the combination of ketamine and SCS resulted in increase of the withdrawal threshold after 30 minutes of SCS, thereby converting these non-responders into responders. The application of the ketamine in combination with SCS is the responders $(n=7)$ did not result in an increase of the withdrawal threshold as compared to SCS alone. The combined use of ketamine and SCS in the responders did however statistically increase the duration of the anti-allodynic effect.

\section{Conclusion}

The combined treatment of SCS and sub-effective dose of i.t. ketamine in nonresponders resulted in a significant reduction of the withdrawal threshold in all previously non-responders to SCS, thereby converting them into responders to SCS. 


\section{Introduction}

Peripheral nerve injury may result in chronic intractable neuropathic pain, which is often associated with tactile hypersensitivity (mechanical allodynia) in patients. An established treatment for these patients is spinal cord stimulation (SCS). In 20$30 \%$ of well selected patients presenting with chronic neuropathic pain, trial SCS is unsuccessful $[99,218,222]$. These patients therefore do not receive a permanent implant of the SCS device and as a consequence do not benefit the long-term pain relief of SCS. These so called "non-responders" to SCS pose a challenge for both clinicians and basic researchers.

In an animal model of neuropathic pain and SCS the percentage of nonresponders to SCS is comparable to that in patients $[197,201]$. In this animal model, SCS results in a decreased release of the excitatory amino acid (EAA) glutamate and an increased release of the inhibitory amino acid (IAA) gammaamino butyric acid (GABA) in the spinal dorsal horn. This is thought to account for the pain relieving effect in responders to SCS [36]. In non-responders however, SCS induces no significant changes in inhibition (GABA) or excitation (EAAs) $[36,206]$. The non-responders were characterized by a more impaired GABAergic system. Potentiation of the impaired GABA-ergic system by the addition of GABA analogs or a low, by itself ineffective dose of a $\mathrm{GABA}_{\mathrm{B}}$ agonist combined with SCS, resulted in potentiation of the pain suppressing effect of SCS [206].

The activation or phosphorylation of the $N$-methyl $D$-aspartate (NMDA) receptor is thought to be the resultant of increased peripheral glutamatergic input, leading to central sensitization which is associated with chronic neuropathic pain $[137,237]$. Hence, interfering with the process of central sensitization through the antagonism of the NMDA receptor may attenuate chronic neuropathic pain. The use of the non-competitive NMDA-blocker ketamine has been shown to have an analgesic effect on neuropathic pain in both experimental and clinical studies $[90,129,185]$. Despite the widely accepted use of ketamine as a third line analgesic, the evidence for the efficacy of ketamine in the treatment of chronic pain is moderate to weak [74]. Furthermore, the dosage of ketamine being used in patients with neuropathic pain to reach relevant pain relief often comes with considerable side effects. Because ketamine and SCS as mono-therapies are hampered by either unsatisfactory pain relief and/or side effects in a subset of patients, we aimed to test whether a low sub-effective dose of intrathecal (i.t.) ketamine could potentiate the pain relieving effect in SCS non-responders. 


\section{Methods}

\section{Animals}

Male Sprague-Dawley rats $(n=22)$, weighing 300-350 g, were used in the experiments. The animals were individually housed under temperature controlled conditions at $21 \pm 1{ }^{\circ} \mathrm{C}$, with a normal 12:12 h light/dark cycle. The animals had free access to food pellets and water.

\section{General}

All experiments were performed in accordance with the European Directive for the Protection of Vertebrate Animals Used for Experimental and Other Scientific Purposes (86/609/EU) and were approved by the Committee for Experiments on Animals of Maastricht, The Netherlands.

Surgery was performed in anesthetized animals under aseptic conditions. Induction and maintenance of general anesthesia were performed by isoflurane inhalation. Constant body temperature was maintained by placing the animal on an automated heating pad $\left(37.5 \pm 0.5^{\circ} \mathrm{C}\right)$, (IDEE, University Maastricht). If signs of extreme weight loss ( $>15 \%$ of body weight at baseline), absence of grooming or appearance of cachectic behavior were observed, the animals were euthanized.

\section{Partial ligation of the sciatic nerve}

A unilateral ligation of the left sciatic nerve was performed as described by Seltzer [187]. The left sciatic nerve was exposed by blunt dissection and carefully freed from surrounding connective tissue. Distal to the posterior biceps semitendinosus, but proximal to the little fat pad that lies a few millimeters distal to this site, the sciatic nerve was partially ligated. An $8 / 0$ non-absorbable silk suture with a reverse cutting micro needle was used to tightly ligate approximately $1 / 3$ to $1 / 2$ of the diameter of the left sciatic nerve. After the ligation, the wound was closed with a 4/0 silk suture.

\section{Intrathecal implantation}

The neck of the animal was shaved and the animal was placed in a stereotactic head holder with the head flexed forward. A midline incision was made at the back of the occipital bone and the neck to expose the atlanto-occipital membrane. The membrane was carefully opened after which a custom made polyethylene catheter (inter diameter $0.20 \mathrm{~mm}$, length $8.5 \mathrm{~cm}$ ) was inserted and tunneled intrathecally to the level of the L3-L5 spinal segments. The wound was closed with 4-0 silk sutures and the catheter was slowly flushed with $10 \mu \mathrm{l}$ of saline. Animals with signs of paralysis directly after the surgery were excluded. 
Correct placement of the catheter was confirmed when lidocaine $2 \%$ injection resulted in paralysis or dragging of the hind limbs.

\section{Implantation of the SCS system}

In the animals with tactile hypersensitivity (mechanical allodynia) and intrathecal catheter $(n=15)$, a SCS system was implanted 14 days after partial ligation of the sciatic nerve. A similar implantation procedure and SCS system were used as reported earlier [197]. Briefly, a rectangular platinum iridium cathode $(3.00 \mathrm{x}$ $1.00 \times 0.10 \mathrm{~mm}$, Medtronic Bakken Research, Maastricht) was implanted epidurally by a small laminectomy at the vertebral T13 level. The wire of the cathode was secured to the spinous process of T12 to prevent movement of the lead. The anode (diameter of $6.0 \mathrm{~mm}$ and thickness of $0.10 \mathrm{~mm}$ ) was placed subcutaneously. Both leads were tunneled subcutaneously to the neck of the animal and connected to micro plugs, which were sutured to the skin of the neck of the animal. Animals were allowed to recover for 2 days after the implantation of the electrodes. For SCS, a Grass S88 stimulator (Astro-med Grass, Warwick, USA) fitted with a Grass SIU-5 stimulus isolator and a Grass constant current unit was used. The stimulus paradigm consisted of the assessment of the motor threshold (frequency $2 \mathrm{~Hz}$, pulse width $0.2 \mathrm{~ms}$ ) followed by $30 \mathrm{~min}$ of SCS (2/3 of motor threshold, frequency $50 \mathrm{~Hz}$, pulse width $0.2 \mathrm{~ms}$ ) as reported earlier $[33,36,180,197]$.

\section{Nociceptive behavioral testing}

All animals were tested using von Frey monofilaments. Testing took place in a set of Plexiglas cages with an elevated wire mesh floor. Prior to testing, the animals were allowed to adapt for 30 min. After acclimatization, the von Frey filaments were applied in an order of increasing stiffness, through the wire mesh floor to the mid-plantar surface of the left and right hind paw until the filaments bent slightly. Each filament was applied 5 times and the minimal value which caused more than 3 brisk withdrawals of the hind paw was recorded as the paw withdrawal threshold (PWT). The animals were tested using 13 von Frey monofilaments (Stoelting CO, IL, USA) with logarithmically incremental stiffness $(0.16,0.4,0.6$, $1.0,1.4,2.0,4.0,6.0,8.0,10.0,15.0,26.0$ and $60.0 \mathrm{~g})$.

\section{Development of tactile hypersensitivity}

Prior to the Seltzer ligation (pre-nerve injury) PWTs were measured of the ipsilateral and contralateral hind paw. Withdrawal thresholds were assessed again at 2 days, 7 days and 14 days post operative (DPO2, DPO7 and DPO14). 


\section{Determination of the individual sub-effective dose ketamine}

In the first experiment, in 6 animals with tactile hypersensitivity, the sub-effective dose of ketamine was determined. Ketamine hydrochloride (Eurovet, Bladel, The Netherlands) was diluted with preservative free, normal saline to a concentration of 50,100 or $200 \mu \mathrm{g} / 10 \mu \mathrm{l}$. Normal saline was used as the negative control. The i.t. injection was followed by a flush of $10 \mu \mathrm{l}$ normal saline $(0.9 \%)$. Ketamine was prepared freshly on the day of testing. Prior to i.t. injection of ketamine $(t=0)$ PWTs were assessed. Each animal received 50, 100, $200 \mu \mathrm{g}$ ketamine or normal saline $(0.9 \%)$ at random on subsequent days (DPO 10, DPO11, DPO 12 and DPO 13). In order to assess whether the dosage of ketamine used could impair motor function, each rat was assessed for the righting reflex and gait [124]. After the injection, PWTs were measured every $15 \mathrm{~min}$ until $120 \mathrm{~min}$ following the injection. The sub-effective dose of ketamine was defined as the dose that did not result in an increase of $\geq 2$ von Frey filaments compared to pre-ketamine PWT during the 120 min testing period.

\section{Spinal Cord Stimulation}

Prior to stimulation (pre-SCS) PWTs were measured. Rats were given continuous SCS for $30 \mathrm{~min}$. Rats were tested 15 and $30 \mathrm{~min}$ following initiation of SCS. After $30 \mathrm{~min}, \mathrm{SCS}$ was terminated and the animals were tested the following 15, 30, 60 and $90 \mathrm{~min}(t=45,60,90$ and $120 \mathrm{~min}$ ). Rats who did not return to pre-SCS PWTs after 90 min were tested once more the next day.

A responder or non-responder to SCS was defined using the maximum possible effect in \% (MPE). An MPE of $100 \%$ represents a return to the pre-nerve injury withdrawal threshold (i.e. no neuropathic pain). A responder to SCS was defined when SCS resulted in an MPE > 50\%. MPE was calculated for each individual animal according to the following formula: MPE \% = (withdrawal threshold after $30 \mathrm{~min}$ of SCS - withdrawal threshold pre-SCS)/(pre-nerve injury withdrawal threshold - withdrawal threshold pre-SCS) x 100\% [215].

\section{Experimental procedure}

All animals with tactile hypersensitivity received $30 \mathrm{~min}$ of SCS. Non-responders to SCS were tested again on the next day. Pre-SCS PWTs were tested, followed by injection of the sub-effective dose i.t. ketamine or normal saline. This was followed by $30 \mathrm{~min}$ of SCS and the PWTs were assessed according to the paradigm described above. The following day each animal received the opposite treatment. The researcher performing the behavioral testing was blinded for the substance injected. Animals that did respond to SCS on DPO 16, were given their sub-effective dose ketamine on DPO 17, followed by 30 min of SCS. 


\section{Statistical analysis}

The withdrawal thresholds to von Frey filaments are presented as mean \pm standard error of the mean (SEM). For statistical analysis of differences in the withdrawal thresholds over time, the non-parametric Friedman test, followed by Dunn's post hoc test, was used. Differences in withdrawal thresholds between groups were compared using the Mann-Whitney test. For analysis of the area under the curve

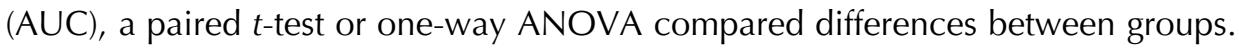
$p<0.05$ was considered to be statistically significant in all tests.

\section{Results}

\section{General observations}

Twenty-two animals displayed tactile hypersensitivity after a unilateral partial ligation of the sciatic nerve. Of the animals $(n=22)$ which received an intrathecal catheter, 1 animal displayed clear paralysis directly after implantation and was excluded. Six of the 21 animals were used to determine the sub-effective dose of ketamine. The remaining 15 animals received $30 \mathrm{~min}$ of SCS + sub-effective ketamine.

\section{Sub-effective dose of ketamine}

In the 6 animals that were used to determine the individual sub-effective dose of ketamine, partial ligation of the sciatic nerve resulted in tactile hypersensitivity After intrathecal injection of saline or $50 \mu \mathrm{g}$ of ketamine, no increase of more than 2 von Frey filaments could be observed for each individual animal over a testing period of $2 \mathrm{~h}$ (Table S6.1, available online) or a significant increase in the withdrawal threshold (Fig. S6.1A, available online).

After the intrathecal injection of $200 \mu \mathrm{g}$ of ketamine a short lasting (approximately 5 min) motor impairment was observed in 4 out of 6 animals. However, the righting reflex and gait were normal after this short period and withdrawal thresholds could be assessed reliably. In all animals $(n=6), 200 \mu \mathrm{g}$ of ketamine resulted in an increase of $\geq 2$ von Frey filaments (Table S6.1, available online) and a significant increase of the PWT of the ipsilateral paw from was noted (Fig. S6.1C, available online). Ketamine did not affect PWT of the contralateral paw (Fig. S6.1C, available online). Intrathecal application of $100 \mu \mathrm{g}$ of ketamine did not result in a statistically significant increase of the ipsilateral PWT (Fig. S6.1B, available online). However, individual data showed that in 3 out of 6 animals an increase of $\geq 2$ von Frey filaments was noted (Table S6.1, available online). From this it was concluded that $100 \mu \mathrm{g}$ of ketamine is an effective dose of ketamine for these 3 animals. For the whole group, the \% MPE of 50, 100 and $200 \mu \mathrm{g}$ was 2.0 $\pm 2.0 \%, 17.2 \pm 8.7 \%$ and $28.4 \pm 4.8 \%$ respectively (Fig. S6.2D, available online), which is comparable to other studies. It was decided that $100 \mu \mathrm{g}$ of 
ketamine should be used to start the determinion of the sub-effective/effective dose for each individual animal in the following experiment. As can be seen in Table S6.2 (available online) in 9 out of 15 animals $100 \mu \mathrm{g}$ of ketamine resulted in an increase of more than 2 von Frey filaments and a significant increase of the PWT of the ipsilateral paw (Fig. S6.2A, available online). Furthermore, the AUC of the 9 animals $(73.5 \pm 7)$ was significantly increased compared to the group where $100 \mu \mathrm{g}$ of ketamine was considered sub-effective $(28.2 \pm 0.8)$ or saline $(19.1 \pm$ 0.6) (Fig. S6.2B, available online). For the 9 animals, the individual sub-effective dose for SCS was therefore set at $50 \mu \mathrm{g}$ of ketamine. For the remaining 6 animals $100 \mu \mathrm{g}$ of ketamine was considered as the sub-effective dose (Table S6.2, available online). The pre-ketamine withdrawal thresholds of the 9 and 6 animals were not significantly different (Fig. S6.2, available online).

\section{Spinal Cord Stimulation in the total population}

The development of mechanical allodynia of the ipsilateral hind paw in the 15 animals that received SCS on DPO 16 is shown in Fig. 6.1A. Two days (DPO 2) after partial ligation of the ipsilateral sciatic nerve a significant decrease of the ipsilateral PWT $(4.9 \pm 0.8 \mathrm{~g})$ compared to pre-nerve injury $(43.4 \pm 4.8 \mathrm{~g})$ was noted $(p<0.001)$. This significantly decreased withdrawal threshold at DPO 2 remained stable up to DPO 14 . No effect of the Seltzer ligation on the PWTs of the contralateral paw could be observed (Fig. 6.1A).
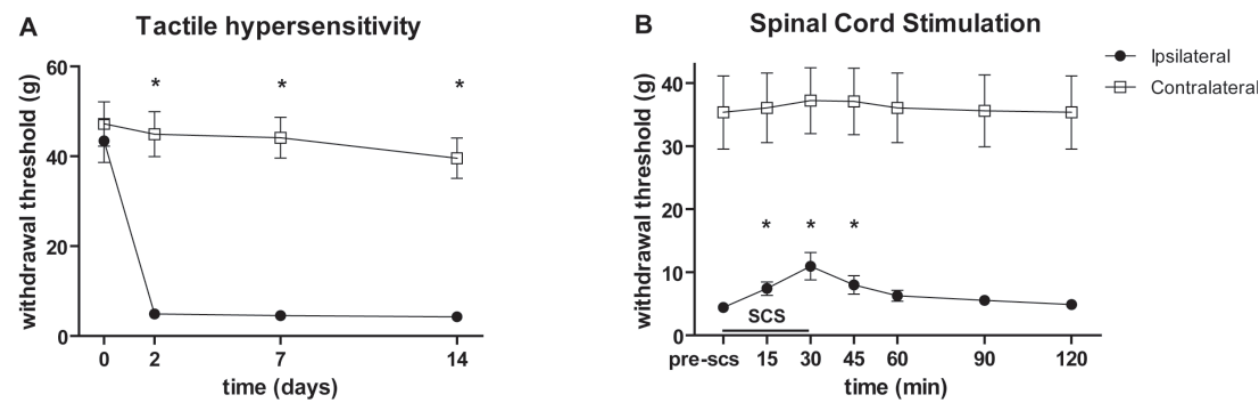

Figure 6.1. The effect of a Seltzer ligation and SCS on PWTs $(n=15)$. (A) After nerve-injury, the PWTs were significantly decreased at DPO2. The decreased PWTs remained decreased up to DPO 14. No effect of the Seltzer ligation on the PWTs of the contralateral paw could be noticed. (B) Thirty minutes of SCS resulted in a significant increase of PWTs of the ipsilateral paw. Already 15 min after the start of SCS, PWTs were increased. This increase remained present $15 \mathrm{~min}$ after termination of SCS $(\mathrm{t}=45$ min). SCS did not affect the PWTs of the contralateral paw. ${ }^{*} p<0.05$ compared to pre-nerve injury or pre-SCS. 
The implantation of the SCS device alone did not affect the PWTs of the ipsi or contralateral hind paw at DPO 16 (pre-SCS PWT of $4.4 \pm 0.8 \mathrm{~g}$ ) as compared to the pre-implantation withdrawal threshold ipsilateral of $4.3 \pm 0.8 \mathrm{~g}(p>0.05)$ at DPO14 (Fig. 6.1A and B). After the start of SCS, the PWT of the ipsilateral paw increased, reaching maximum values after $30 \mathrm{~min}$ of SCS $(t=30 \mathrm{~min})$ (Fig. 6.1B). After 30 min of SCS, the ipsilateral PWT $(11.0 \pm 2.2 \mathrm{~g})$ was significantly increased compared to the PWT at pre-SCS $(4.4 \pm 0.8 \mathrm{~g}),(p>0.05)$. After termination of SCS, PWTs started to slowly decrease, returning to pre-SCS values at $t=60 \mathrm{~min}$ (Fig. 6.1B). No effect of SCS on the PWT of the contralateral paw was noted.
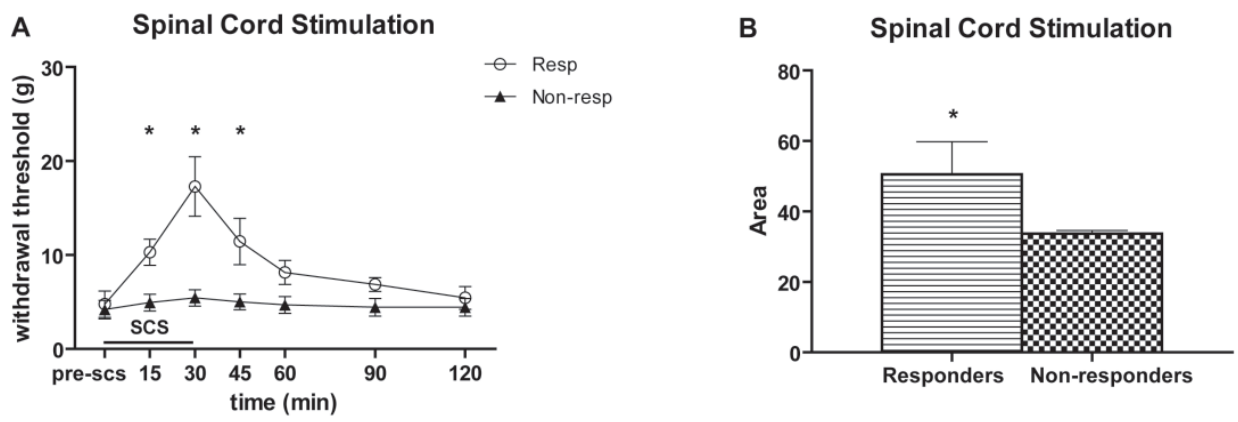

Figure 6.2. Responders $(n=7)$ and non-responders $(n=8)$ to SCS based on an MPE $>50 \%$. (A) Each individual responder to SCS showed a MPE $>50 \%$. In these animals, 30 min of SCS resulted in a significant increase of the PWTs of the ipsilateral paw, starting already 15 min after the start of SCS. After termination of SCS, PWTs started to decrease, but remained significantly increased compared to non-responders until $t=90 \mathrm{~min}$. In the non-responders (MPE $<50 \%$ ), no significant increase of the PWTs of the ipsilateral paw were detected. (B) The AUC of the responders to SCS was significantly increased compared to the AUC of the non-responders. ${ }^{*} \mathrm{p}<0.05$ compared to pre-SCS.

\section{Non-responders and responders to SCS}

Next, we calculated the MPE after $30 \mathrm{~min}$ of SCS for each individual animal, using the definition of a responder to SCS (MPE >50\%). Out of the 15 animals that received SCS, 8 animals were defined as non-responders to SCS (Table S6.3, available online). These animals did also not show a significant increase of the ipsilateral PWTs after 30 min of SCS (Fig. 2A). Of the remaining 7 animals, which were defined as responders, $30 \mathrm{~min}$ of SCS resulted in an MPE of $72 \pm 6 \%$ (Table S6.3, available online). In these responders, already $15 \mathrm{~min}$ after the start of SCS, PWTs $(10.3 \pm 1.4 \mathrm{~g})$ were significantly increased $(p=0.01)$ as compared to preSCS PWTs $(4.8 \pm 1.4 \mathrm{~g})$. The maximum increase of the PWT $(17.3 \pm 3.2 \mathrm{~g})$ was reached $30 \mathrm{~min}$ after the start of SCS. Fifteen minutes after termination of SCS $(t=$ $45 \mathrm{~min})$, PWTs remained significantly increased $(11.4 \pm 2.5 \mathrm{~g})(p=0.001)$ as 
compared to pre-SCS PWTs. The responders and non-responders to SCS did not differ with respect to the pre-SCS PWTs $(4.8 \pm 1.4 \mathrm{~g})$ and $(4.2 \pm 1.0 \mathrm{~g})$ respectively $(p>0.05)$.

The difference in response to SCS is also evident in the AUC, where the AUC of the responders to SCS $(50.6 \pm 9.14)$ was significantly increased compared to the AUC of the non-responders $33.7 \pm 0.9$ ( $p<0.05$ ) (Fig. 6.2B).

No difference in the response to intrathecal ketamine $(100 \mu \mathrm{g})$ alone could be observed between responders and non-responders to SCS.

\section{Non-responders to SCS + sub-effective dose of ketamine}

For each SCS non-responder the combination of the sub-effective dose of ketamine and SCS resulted in an MPE > 50\% thereby qualifying them as responders to SCS. When normal saline was injected, the MPE for each individual animal never reached $>50 \%$ (Table S6.3, available online). Furthermore, 30 min after the initiation of SCS + sub-effective ketamine, maximum PWTs were reached $(25.5 \pm 5.5 \mathrm{~g})$ with a significant increase compared the pre-SCS PWTs $(4.9 \pm 0.8$ g) $(p<0.001)$. This significant increase remained detectable $30 \mathrm{~min}$ after termination of SCS (Fig. 6.3A), which is increased to the responder group (Fig. 6.2A). No increase of the PWTs could be observed after saline injection (Fig. 6.3A). The AUC of the SCS + sub-effective dose of ketamine $(103 \pm 22)$ was significantly increased compared to the AUC of the saline + SCS $(36 \pm 1.1)(p<$ 0.001) (Fig. 6.3B). SCS + sub-effective ketamine did not affect the withdrawal thresholds of the contralateral paw (Fig. 6.3B).
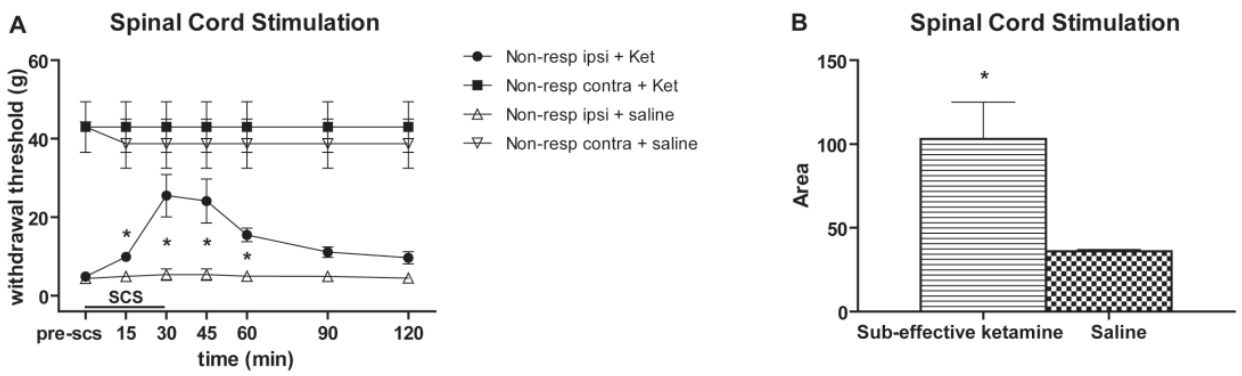

Figure 6.3. Combination of SCS and the individual determined sub-effective dose of i.t. ketamine in non-responders $(n=8)$. (A) After injection of ketamine, SCS resulted in a significant increase of the ipsilateral PWTs, already noticeable $15 \mathrm{~min}$ after the start of SCS. The increased PWTs were still significantly increased 30 min after termination of SCS $(t=60)$ When normal saline was injected in the same animals, no effect on the PWTs could be detected. Again, the combination of SCS and ketamine or saline did not affect the PWTs of the contralateral paw. (B) The AUC of the SCS + ketamine was significantly increased compared to the AUC of the SCS + saline. * $p<0.05$ compared to pre-SCS values. 


\section{Responders to SCS + sub-effective dose of ketamine}

In 2 of the 7 the responders to SCS ketamine could not be injected due to obstruction of the catheter (Table S6.3, available online). Therefore 5 responders received the individual determined sub-effective dose ketamine + SCS. In 4 out of the 5 animals, the addition of the sub-effective dose of ketamine to SCS resulted in an increase of the MPE (Table S6.3, available online), although there was no overall significant increase of the MPE compared to the MPE of SCS per se $(87 \pm 8$ $\%$ and $72 \pm 6 \%$ respectively) ( $p>0.05$ ). Furthermore, the maximal PWT reached after 30 min of SCS was comparable between SCS per se and SCS + sub-effective ketamine (Fig. 6.4A). The sub-effective dose of ketamine however resulted in an extended duration of the increased PWT of the ipsilateral paw (Fig. 6.4A). With the addition of the sub-effective dose of ketamine to SCS, the significantly increased PWTs could still be noticed 90 min after termination of SCS $(9.6 \pm 0.4$ g) $(t=120 \mathrm{~min})$ as compared to pre-SCS PWT $(2.6 \pm 0.9 \mathrm{~g})(p=0.003)$. This is also depicted in Figure 6.4B, where the AUC of SCS + sub-effective ketamine is significantly increased to the AUC of SCS per se. The effect of SCS per se dissipated already $30 \mathrm{~min}$ after termination of SCS. At SCS 60 min PWT was $8.0 \pm$ $1 \mathrm{~g}$ which was not statistically increased compared to PWT pre-SCS $(5.0 \pm 1 \mathrm{~g})(p$ $>0.05$ ) (Fig. 6.4A).
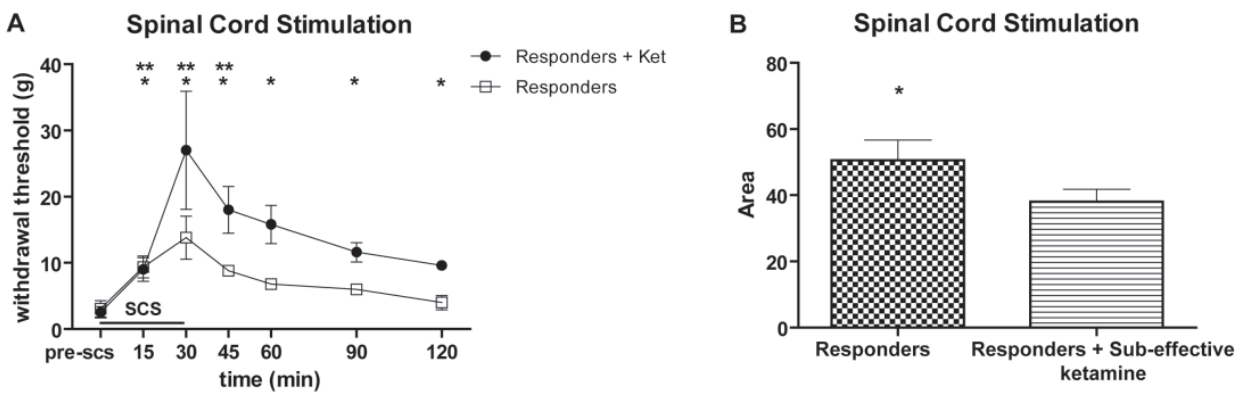

Figure 6.4. Combination of SCS and the individual determined sub-effective dose of i.t. ketamine in responders to SCS per se $(n=7)$. (A) After injection of ketamine, SCS resulted in an increase of the ipsilateral PWTs. This increase was comparable to the increase of the PWTs after SCS alone. After termination of SCS however, PWTs decreased more slowly as a result of the addition of ketamine as compared to SCS per se. Indicating prolonged analgesia as a result of SCS. (B) The AUC of the SCS + ketamine was significantly increased compared to the AUC of the SCS per se. ${ }^{*} \mathrm{p}<0.05$ compared to pre-SCS + ketamine Responders, ** compared to pre-SCS for SCS per se. 


\section{Discussion}

The present results demonstrated that administration of a single sub-effective dose of ketamine i.t. in non-responders to SCS significantly enhanced the pain relieving effect of SCS on the tactile hypersensitivity, converting all these animals into responders. Furthermore, the duration of the anti-allodynic effect of SCS was extended in the responders and non-responders to SCS after the combination with a sub-effective dose of ketamine i.t.

The activation, or phosphorylation, of the NMDA receptor is thought to play an important role in the induction and maintenance of neuropathic pain and ketamine is able to reduce neuropathic pain in both patients and animal models $[55,90,220,243]$. In the present study, the individual sub-effective dose of intrathecally applied ketamine was determined to evaluate the spinal site of action of ketamine and to minimize side effects. We reported a tailor made sub-effective dose per individual animal of 50 or $100 \mu g$ ketamine. With these concentrations no side effects could be observed, such as motor impairment or sedation. Furthermore in animals where $100 \mu$ g i.t. ketamine was effective in attenuating the neuropathic pain no anti-nociception in the contralateral paw could be observed, which is in line with previous studies $[126,129]$.

There is both neuronal, neurochemical and electrophysiological evidence suggesting that the behavioral effects of SCS are mediated at the level of the spinal cord $[113,135,196,231]$ resulting from a change in the concentration of neurotransmitters in the spinal cord [36]. Modulating the EAA concentration, for instance by SCS, will therefore indirectly influence receptor activation in the dorsal horn. Electrical neuromodulation in a chronic visceral hypersensitivity model resulted in a decreased phosphorylated sub-unit of the NMDA receptor (NR1) in the dorsal horn [211]. Here it was suggested that the pain relief observed was the resultant of decreased release of EAA resulting in a decreased phosphorylation of the NMDA NR1 subunit. Furthermore, the pre-synaptic release of glutamate and aspartate is known to be under GABA-ergic control [84] and the SCS induced decrease of EAA could be blocked by the GABA ${ }_{B}$ receptor antagonist Baclofen [36]. In animals with chronic neuropathic pain that did not respond to SCS however, both levels of EAAs and GABA were unaffected by SCS. Besides the modulation of EAAs and GABA, serotonin and acetylcholine have been shown to be involved in the SCS induced analgesia [180,201].

The use of NMDA receptor antagonists, like ketamine, is an example of direct modulation of the activity of this receptor. It should be noted that ketamine exerts its effect not exclusively through modulation of the NMDA receptor. For instance, it was shown that intrathecal ketamine impaired excitability of nociceptivespecific neurons by blocking sodium currents [184] and tetrodotoxin sensitive and resistant sodium channels in the rat dorsal root ganglion (DRG) [245]. In this respect it is important to note that the sub-effective dose of i.t. ketamine we used in the current experiment did not induce any observable behavioral effect. It can 
however not be excluded that i.t. administered ketamine reaches the DRG and through modulating the Na-channels partially inhibits DRG excitability. This partial inhibition may also be involved in the pain relieving effect as observed after combined treatment of i.t. ketamine and SCS. Furthermore, it was reported that the NMDA receptor antagonist (AP7) i.t. reduced glutamate release in an animal model of acute arthritis in the rat, suggesting that glutamate release depends on NMDA activation [194]. Interestingly, activation of pre-synaptic NMDA receptors was shown to inhibit and delay the synaptic release of glutamate in the rat spinal cord [7]. Although the underlying mechanisms of the combination of SCS and ketamine remain unclear, our data strongly suggest a critical role of the NMDA receptor in the conversion of non-responders to SCS responders. Future molecular and biological experiments need to be performed to further analyze the mode of action of the combination of SCS and ketamine. Our results justify a clinical trial on the combined treatment of SCS and sub-effective dose ketamine in patients with chronic neuropathic pain who do not respond to SCS trial stimulation.

The use of a subanaesthetic dose of ketamine as an analgesic drug is being appreciated in a wide range of pain settings [224]. When applied parenterally (intravenous, subcutaneous, intramuscular, epidural), the evidence in successful pain relief is moderate to weak [74] and side effects such as sedation, dizziness and hallucinations are often reported. The efficacy of oral administration of ketamine in chronic pain also lacks substantial evidence, probably due to the insufficient amount of studies available [15]. Promising results were reported by Finch, reporting decreased allodynia and no side effects after topical application of ketamine [56].

The present experimental study describes a combination of intrathecal ketamine and SCS. In the literature, there is still controversy as to the safety of intrathecal ketamine $[17,50,123]$. In neonatal rats (age 3 days) it was shown that 30 or 100 $\mu \mathrm{g}$ of i.t. ketamine resulted in spinal neuronal apoptosis and glial activation whereas in older rat pups (age 21 days), no apoptosis or glial activation could be seen with a dose of $900 \mu \mathrm{g}$ of i.t. ketamine [229]. It appears that spinal apoptosis through i.t. ketamine is age and dose dependant. With respect to our experiment, the given dose of ketamine $(50-100 \mu \mathrm{g})$ was considerably lower and our rats were clearly older and thus apoptosis or glial activation is unlikely. Nevertheless, from a clinical point of view it should be stressed that i.t. administration of ketamine is not desirable and therefore different routes of administration have to be considered. However, the present results are a proof of principle that modulation of the NMDA receptor through the use of a single low dose of ketamine can increase the efficacy of SCS in animals with neuropathic pain. Interestingly, it was reported that 4 days of intravenous infusion of ketamine (average infusion $\pm 20 \mathrm{mg} / \mathrm{h}$ ) was able to relieve neuropathic pain for up to 10 weeks in CRPS-1 patients [192]. Although effective in pain relief, side effects such 
as nausea, vomiting, psychomimetic effects and headaches were frequently reported [192].

Our data further suggest that a sub-effective dose of ketamine prolonged SCS induced pain relief. Two syndromes with chronic neuropathic pain, CRPS and failed back surgery syndrome (FBSS), have been shown to benefit substantially from SCS [85,155]. The effect of SCS in patients with CRPS-I was reported to diminish over time [87] and after 3 years of SCS, the pain relieving effect of SCS was not significantly different compared to the control group [87]. North and others reported a satisfactory pain relief in patients with FBSS receiving SCS for 23 years [100,156], although recent work could not find any difference between SCS and alternative treatments after 6 months in patients with FBSS [218].

It might therefore be of clinical interest to use a low dose of intravenous ketamine in the group of patients which do not respond to the trial SCS stimulation. The dose ketamine i.v. should preferably be under $20 \mathrm{mg} / \mathrm{h}$ due to reported side effects (Sigtermans et., 2009). Furthermore, in the group of patients that do respond to SCS, but where the effect is slowly diminishing over time (Kemler te al., 2006), additional ketamine treatment might be considered in order to prolong the pain relieving effect of SCS.

In summary, in an animal model of chronic neuropathic pain, the combination of i.t. sub-effective dose of ketamine and SCS potentiates the pain relieving effect in SCS non-responders, thereby converting them into SCS responders. Furthermore this combined therapy prolonged the pain relieving effect in SCS responders. 


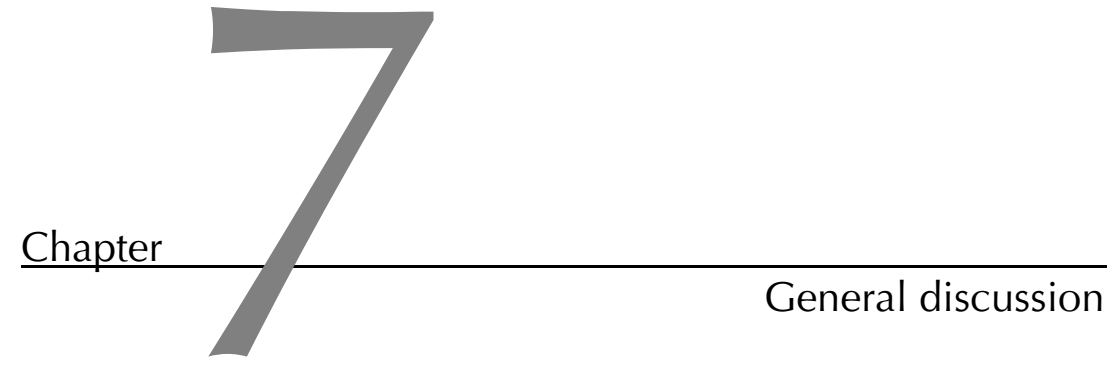



The overall aim of this thesis was to develop and use an experimental mouse model of chronic neuropathic pain and spinal cord stimulation and to explore the possibility to improve the pain relieving effect of spinal cord stimulation.

The use of experimental animal models is of great value for the better understanding of the underlying mechanisms of disease. This better understanding provides us with a tool to improve or create therapies. However, translating data from animal models to the clinic has proven to be of limited success [141]. Therefore, there is still the need for new or improved animal models. In particular when genetic aspects are or may become involved in the cause of the disease, like neuropathic pain, or on the mechanisms underlying the therapy, like SCS, mouse models are favourable as compared to the rat. At present many knock-in, knock-out and overexpressor mice, also related to genes involved in neuropathic pain, are available $[150,154,202,233]$. For chronic neuropathic pain, the need for the development of new or improved therapies is imperative, as $30-40 \%$ of patients with neuropathic pain do not achieve adequate pain relief [57]. This thesis focussed on two aspects, the first being the ability to realize a mouse model of CRPS-1 with behavioral signs of neuropathic pain (chapter 2) and spinal cord stimulation (chapter 3 and 4) and secondly, to use early spinal cord stimulation (chapter 5) and ketamine-enhanced spinal cord stimulation (chapter 6) to improve SCS in a rat model of neuropathic pain.

The first research question ( $1 \mathrm{a}$, chapter 2 ) focussed on the possibility to develop a mouse model of CRPS-1 using a L5 ventral root transection. This model, initially developed in the rat [107], shares similarities with CRPS-1 patients because there are clear signs of neuropathic pain, although there is no evident damage to sensory neurons. In contrast to the findings reported in the Sprague-Dawley (SD) rat, where significant tactile hypersensitivity and thermal hyperalgesia were observed after a L5 VRT [107], our findings did not reveal any sign of sensitivity to tactile stimuli in the C57BL/6 mouse. Although the CatWalk, which is thought to detect small pain induced gait changes as the result of damage to peripheral nerves, could detect gait changes after the L5 VRT in the C57BL/6 mouse, these changes could not be attributed to neuropathic pain. Because it has been reported that the C57BL/6 mouse is moderately sensitive and the 129J mouse is highly sensitive for the development of neuropathic pain after peripheral nerve damage [144], the 129] mouse was included in the L5 VRT study. Although more sensitive, the 129J mouse did not displayed signs of neuropathic pain as measured with the von Frey monofilaments or the CatWalk after a L5 VRT. A recent report on the L5 VRT in C57BL/6 mouse describes a marked increase in Nav 1.3 and Nav 1.8 in the DRG [72]. Unfortunately, the upregulation of these sodium channels were not substantiated by behavioral data on neuropathic pain. Indeed, the upregulation of the Nav 1.3 and Nav 1.8 sodium channels in the DRG are associated with neuropathic pain [11,172], although the upregulation of Nav 1.3 and Nav 1.8 in the DRG do not seem to be a prerequisite for the development of neuropathic pain. Taken together, changes in neuropathic pain related ion- 
channels do not always result in behavioral signs of neuropathic pain, suggesting an important, but not crucial role, for the development of neuropathic pain.

Furthermore, it should be noticed that a difference in anatomy between mice and rat exists. In the rat as in the mouse the L3, L4 and L5 DRG contribute to the sciatic nerve, but the contribution of the L5 in rat is more prominent [171]. Hence, transection of the L5 ventral root in mice might result in indirect inflammation of the L5 DRG, but because of its minimal contribution to the sciatic nerve, behavioural signs are difficult to detect. This may, to some extent, explain the discrepancy between behavioral signs of neuropathic pain in mice and rats after an L5 VRT. The question remains which mouse model is an appropriate model for CRPS-1. Besides the L5 VRT model, two other models of CRPS-1 have recently been described in rats. One model is based on the observation of the high incidence of CRPS-1 in patients after a distal tibial fracture and the second model is based on the evidence that CRPS-1 may depend in part to tissue ischemia. The distal tibial fracture rat model exhibits hindpaw warmth, edema and signs of neuropathic pain [64]. These signs were attributed to substance $P$ induced increased vascular permeability (increased protein extravasation). Interestingly, the same role for substance $P$ signalling in the vascular and nocicieptive changes was observed in a model of CRPS-2 [93]. The second model, the Chronic Post Ischemia Pain (CPIP) rat model, is based on a 3 hours ischemia and reperfusion of the hind paw [28]. The CPIP model also exhibits hindpaw warmth, edema and signs of neuropathic pain and increased protein extravasation. Behavioral signs of neuropathic pain could be attenuated by the free radical scavengers NAC and Tempol. Furthermore, the CPIP model showed no microscopic evidence of nerve damage 48 hours after the ischemia-reperfusion could be observed. The CPIP model has recently successfully been transferred to the mouse [138].

Because the L5 VRT in mice proved unsuccessful for the development of neuropathic pain (chapter 2), we used another neuropathic pain model, namely the Seltzer model, or the partial ligation of the sciatic nerve. This model has been used in $\mathrm{C} 57 \mathrm{BL} / 6$ mice for the induction and maintenance of neuropathic pain [125] and therefore may be used to test the effect of spinal cord stimulation on mechanical sensitivity in mice. Furthermore, the Seltzer model in the rat has successfully been used in combination with SCS in Maastricht [197].

Within the first objective, the second research question (1b, chapter 3 and 4) addressed the possibility of implementing a spinal cord stimulation device in C57BL/6 mice after a partial ligation of the sciatic nerve (Seltzer ligation) [187]. Chapters 3 and 4 report that, with technical adaptations to the SCS device, it was possible to use the mouse model both for inducing neuropathic pain and to test the effect of SCS. The development and maintenance of neuropathic pain (tactile hypersensitivity) was comparable to that previously seen in Sprague-Dawley (SD) rats [197]. The analgesic effect of SCS seen in C57BL/6 mice however differed to 
that observed in SD rats. First, the percentage of responders to SCS in C57BL/6 mice was increased $(100 \%)$ as compared to the responders to SCS in SD rats (approximately $50 \%$ ). Second, SCS in C57BL/6 mice with neuropathic pain resulted in a return to pre-nerve injury withdrawal thresholds whereas in SD rats, SCS results in an increase of about $30 \%$ of pre-nerve injury withdrawal thresholds [197].

The results as presented in chapter 2, 3 and 4 have led to the following conclusions. First, neuropathic pain models in rat, as for instance the L5 VRT model, cannot automatically be transferred to the mouse. On the other hand, the partial ligation of the sciatic nerve (Seltzer) did result in similar changes in withdrawal thresholds as measured with the von Frey in mice and in rats (chapter 3 and 4). Second, the effect of SCS in mice with neuropathic pain clearly differed from that observed in rats. The differences noted between rat and mouse models in itself may result in new insights on the pain relieving effect of SCS. Furthermore, the implementation of SCS in the mouse with neuropathic pain does allow for future molecular-genetic studies on the mechanisms of SCS.

With respect to the measurement of "pain" or analgesia in animal models of neuropathic pain induced by partial ligation of the sciatic nerve (Seltzer), both the von Frey monofilaments and the CatWalk were used (chapter 2 and 4). From the results in chapter 4 it became evident that changes in behaviour after the Seltzer ligation or after SCS were more prominent and long lasting with the use of the von Frey monofilaments. The CatWalk parameters Intensity, Stance and Swing duration were significantly decreased after the Seltzer ligation, however this decrease dissipated over time and was not as robust as the decrease of the von Frey withdrawal thresholds. Furthermore, no changes of the CatWalk parameters were noted after SCS, whereas the von Frey withdrawal thresholds dramatically increased after SCS. As suggested that "the CatWalk method might detect small changes in mechanical sensitivity that are not detectable by von Frey probing" [226], the CatWalk could be useful in chapter 2, where no changes in tactile withdrawal thresholds using the von Frey method could be observed after the L5 VRT in two strains of mice. However, the CatWalk gait analysis did not allow to detect any changes induced by indirect inflammation of the peripheral sensory system. It should be taken into account that the CatWalk detects and records gait and gait is both motor and sensory dependent. Changes in gait as the result from damage to the peripheral nerve may be difficult to interpret. Indeed, the Seltzer ligation induces damage to both sensory and motor nerve fibers and therefore gait changes recorded by the CatWalk cannot automatically be attributed to "pain" and may as well be the result of motor nerve damage. Moreover, the results from chapter 4 and the results that gabapentin in mice with neuropathic pain do not affect CatWalk parameters [143] strongly suggests that CatWalk monitored gait changes may not be related to pain.

It is generally accepted that the von Frey method also has its limitations, in particular in view of the clinical assessment of neuropathic pain, where the von 
Frey only measures hypersensitivity to innocuous stimuli. Obviously in the clinic, patients and the effect of SCS are defined based on spontaneous pain responses. Indeed, the fact that results from basic pain research do not always result in clinical improvements has led to the question whether the current methods for measuring experimental pain related behavior can be improved [141]. It was proposed that spontaneous pain behavior as present in neuropathic pain, such as hypolocomotion, flinching, licking, and guarding of the affected limb, might be a better approach in experimental pain research. Mogil and collogues assessed the above mentioned spontaneous pain behaviors in 22 genetically different strains of mice after a spared nerve injury (SNI) and compared this with the conventional von Frey paw withdrawal response [143]. It was concluded that the behaviors associated with spontaneous pain are not appropriate measures of neuropathic pain in mice. Furthermore, the same study showed that changes in gait as measured with the CatWalk displayed a different time course as that of the changes in von Frey measurements. Taken together, spontaneous pain behaviours in animals might best reflect "pain" in patients, however, with the lack of good tools for the measurement of spontaneous pain in the experimental setting, the von Frey is the "next best tool".

In the second part of this thesis, we aimed at investigating approaches to improve the efficacy of the pain reducing effect of SCS in the rat model of chronic neuropathic pain and SCS. Although SCS is successful in patients with chronic neuropathic pain, still a considerable amount of patients do not receive a permanent implant and furthermore, the effect of SCS is retreating over time. From previous work, it became apparent that the effect of SCS in rats with neuropathic pain shows similarities with those observed in patients [36,197] where the increased success of drug-enhanced SCS in rats was also reported to increase the success of SCS in patients [109,179].

In line with this translational research we showed that SCS after $24 \mathrm{~h}$ of neuropathic pain (early SCS) results in a increased efficacy as compared to SCS applied after 16 days (late SCS) of neuropathic pain in rats (chapter 5). It is important to realize that "increased efficacy" can have different interpretations. First, the increased efficacy may implicate an increased number of animals with pain relief. Second, an increased efficacy may also be concluded when increased withdrawal thresholds, or improved pain relief per individual animal is being achieved. The experiment in chapter 5 demonstrated that the increase of the withdrawal threshold as a result of early SCS was comparable to that of late SCS and hence early SCS does not result in improved pain relief. On the other hand, early SCS was shown to be more effective as compared to late SCS because early SCS resulted in pain relief in $77 \%$ of the animals as compared to $38 \%$ in the animals receiving late SCS (chapter 5). These results suggests that the timing of the intervention is important for the success of pain relief in neuropathic pain. It was reported that decreased GABAergic inhibition in neuropathic pain is time 
dependent $[48,78]$ and early intrathecal GABA results in better pain relief than late intrathecal GABA application in CCI rats [47]. As SCS exerts its pain relieving effect in part through the GABAergic system, early SCS might be more effective by modulating the GABAergic system in an early phase in the development of neuropathic pain. On the other hand, NMDA receptor activation and subsequent central sensitization might not be as robust in an early phase of neuropathic pain as compared to 16 days after nerve injury or late phase.

It is common use for human therapeutical interventions to define "clinical relevant pain relief". For SCS in patients with neuropathic pain, this definition is normally set at a $50 \%$ decrease of the VAS as compared to the VAS prior to SCS. To this extent, we defined relevant pain relief after SCS in rats with neuropathic pain when a maximum possible effect (MPE) of $>50 \%$ was reached, which shows similarities with the definition used in patients. In this context, it is important to note that the MPE is based on a decrease of the tactile hypersensitivity, whereas the VAS score quantification is based on a decrease in spontaneous pain. With the use of the MPE in the evaluation of our results (chapter 5 and 6), early SCS results also in an increased number of animals showing relevant pain relief (responders to SCS) as compared to late SCS. Although we do not know the mechanisms responsible for this increased efficacy, our data suggest that an early intervention (by means of SCS) in the process of central sensitization can improve the success of SCS in neuropathic pain. Indeed, several case studies reported an improved outcome when SCS was applied at an early stage of the disease $[71,178,223]$. Our data suggest that early clinical intervention in the process of neuropathic pain will increase the number of responders to SCS and thus from a clinical point of view, early SCS will increase the number of responders to trial SCS, thereby increasing the number of patients receiving adequate pain relief.

Another approach to improve the efficacy of SCS in the rat, which was studied in chapter 6, was the combination of a low dose of intrathecal ketamine, that by itself does not result in pain relief (sub-effective dose) and ineffective SCS (nonresponders). This combination resulted in successful, relevant pain relief, using the MPE > $50 \%$ as the definition for successful/relevant pain relief (Chapter 6 ). From the literature $[33,36,112,113]$ behavioral signs of SCS induced pain relief are correlated with changes in extracellular neurotransmitters in the spinal cord dorsal horn in animals with neuropathic pain. The changes in extracellular neurotransmitters are characterized by a decrease of excitation related neurotransmitters (glutamate) and an increase of the inhibition related neurotransmitters (GABA) in responders to SCS (Figure 7.1A). If SCS did not resulted in changes in behaviour (non-responders to SCS), no changes in the extracellular excitation or inhibition related neurotransmitters could be observed $[36,201,206]$. From this, it was concluded that the GABAergic system in the nonresponders is probably more impaired and thereby unable to respond (with increased GABA release) to SCS. Assisting the impaired GABAergic system in non- 
responders by means of intrathecal application of GABA analogues (gabapentin and pregabalin) or a GABA receptor agonist (baclofen) in combination with SCS proved to decrease the numbers of non-responders to SCS. Interestingly, the drugs mentioned above are by itself ineffective (sub-effective dose) in alleviating the neuropathic pain, as is SCS (non-responders). Combining the two ineffective therapies now showed effectiveness in treating neuropathic pain $[35,231]$.

The primary effect of SCS has been attributed to the antidromic activation of ascending dorsal column fibers inducing segmental neurochemical changes in the dorsal horn as discussed above. However, there is evidence that an orthodromic activation occurs involving supraspinal mechanisms via a loop in which the descending dorsolateral funiculus (DLF) is involved in the attenuation of neuropathic behavioural signs by SCS $[53,169,174]$, therefore both spinal and supraspinal mechanisms appear to be involved in the pain relieving effect of SCS.

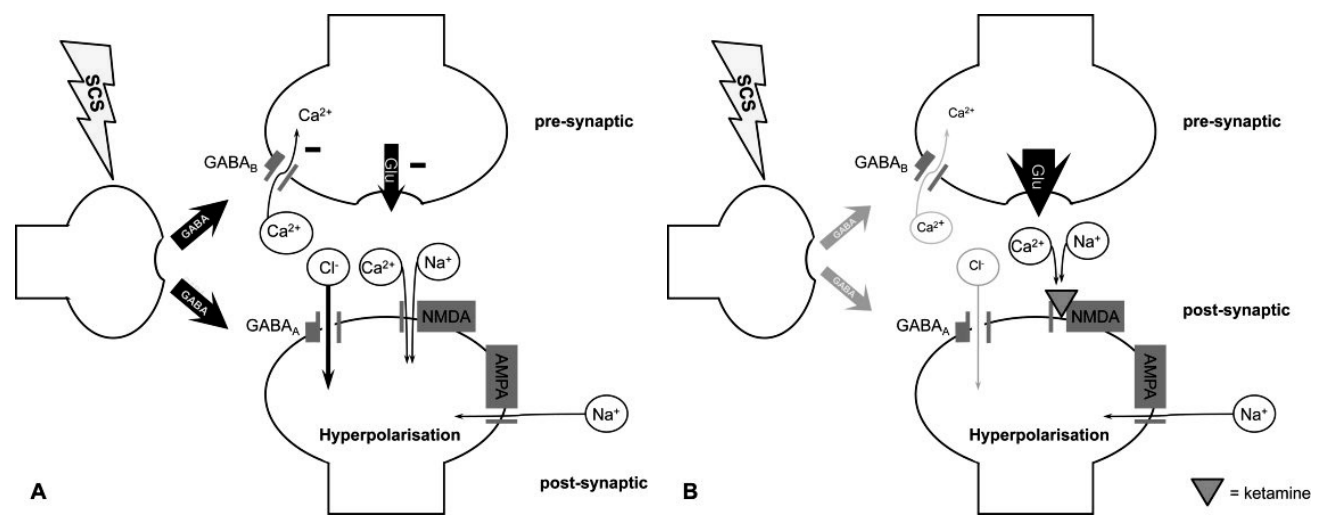

Figure 7.1 (A) SCS in responders. SCS results in an increased extracellular GABA concentration. GABA exerts its effects pre-synaptically via $G_{A B A_{B}}$ receptors through the increase in pre-synaptic $\mathrm{Ca}^{2+}$ concentration, thereby decreasing the glutamate release. Post-synaptically, GABA induces postsynaptically, via the $\mathrm{GABA}_{\mathrm{A}}$ receptor, an increased $\mathrm{Cl}^{-}$concentration. The latter results in a hyperpolarisation of the post-synaptic membrane and subsequently in less neuropathic pain . (B) SCS in non-responders. SCS does not result in a significant increase of extracellular GABA concentration and thus both the modulation of the glutamate release pre-synaptically and the hyperpolarisation postsynaptically is not effective and consequently no reduction in neuropathic pain is noted. Pharmacotherapeutic intervention through the application of ketamine results in a block of the NMDA receptor and thereby a reduction in post-synaptic $\mathrm{Ca}^{2+}$ influx. This results in decreased phosphorylation of both NMDA and AMPA receptors. At the end the effect of increased extracellular glutamate levels as present in SCS non-responders is minimized through the application of ketamine, finally resulting in less neuropathic pain (SCS responder).

With respect to the underlying mechanisms of action of the combined treatment of non-responders by means of intrathecal ketamine and SCS, we propose that, 
although no significant changes in extracellular amounts of glutamate or GABA could be observed [36], still a small decrease in these EAAs and increase in IAAs might occur. However this decrease is too small to result in a decrease in pain related behavioral. Through the blocking of the NMDA receptor in the dorsal horn with the use of a low sub-effective dose of ketamine and the small decrease/increase of EAAs/IAAs by SCS, this might be just enough to result in decreased pain related behaviour (Figure 7.1B). Although the main target for ketamine is the NMDA receptor, it is worth mentioning that ketamine might indirectly exert its analgesic effect through the release of analgesia related neurotransmitters (ie acetylcholine, serotonin) $[2,79,110]$.

The increase of extracellular IAAs in the dorsal horn have been related to the pain relieving effect of SCS. However, there is a discrepancy between the increase of extracellular GABA in the dorsal horn and the anti-allodynic effect of SCS $[36,206]$. During SCS itself, only a slight increase of the extracellular GABA levels was reported, where a clear effect in behavior could already be observed [36]. This is thought to be attributed to binding of released GABA to unoccupied receptors or the rapid reuptake of GABA [36]. Furthermore, a delay caused by the time required for diffusion of GABA from terminals located at a distance from the collecting probe might explain the discrepancy between extracellular GABA and behaviour [206].

On the other hand, other mechanisms underlying the direct and/or prolonged effect of SCS might, in part, contribute to the pain relieving effect. We therefore, hypothesize that as in our experimental settings the prolonged effect of SCS on neuropathic pain might be explained by a form of long term depression (LTD) or depotentiation of dorsal horn neurons. It is known that in the CA1 region of the hippocampus, the strength of excitatory transmission can be both increased and decreased by patterns that activate postsynaptic NMDA receptors (review Malenka and Nicoll 1993). Through high (100 Hz) frequency electrical stimulation of the hippocampus, synaptic transmission undergoes a persistent enhancement known as long term potentiation (LTP) [14]. LTP dependents on a process known to be similar to central sensitization as noted in the dorsal horn of the spinal cord in neuropathic pain. It is believed that both LTP and LTD at synapses in various brain regions require elevation of free cytosolic $\mathrm{Ca}^{2+}$ concentrations via activation of NMDA receptors $[14,111]$. The absolute level of intracellular $\mathrm{Ca}^{2+}$ determines whether synaptic efficacy increases (large elevation of $\mathrm{Ca}^{2+}$ ) or declines (moderate to small increase in $\mathrm{Ca}^{2+}$ ) $[115,153]$. The absolute intracellular $\mathrm{Ca}^{2+}$ concentrations may differentially activate phosphoprotein kinases (high $\mathrm{Ca}^{2+}$ ) and protein phosphatises (lower $\mathrm{Ca}^{2+}$ concentrations). Typically, LTP results from high frequency stimulation $(100 \mathrm{~Hz})$, however, several reports have now confirmed that also low frequency stimulation $(1-5 \mathrm{~Hz})$ in the 
amygdala and hippocampus formation results in LTP $[104,210]$. The same phenomena has been reported for high or low frequency induced LTD [21].

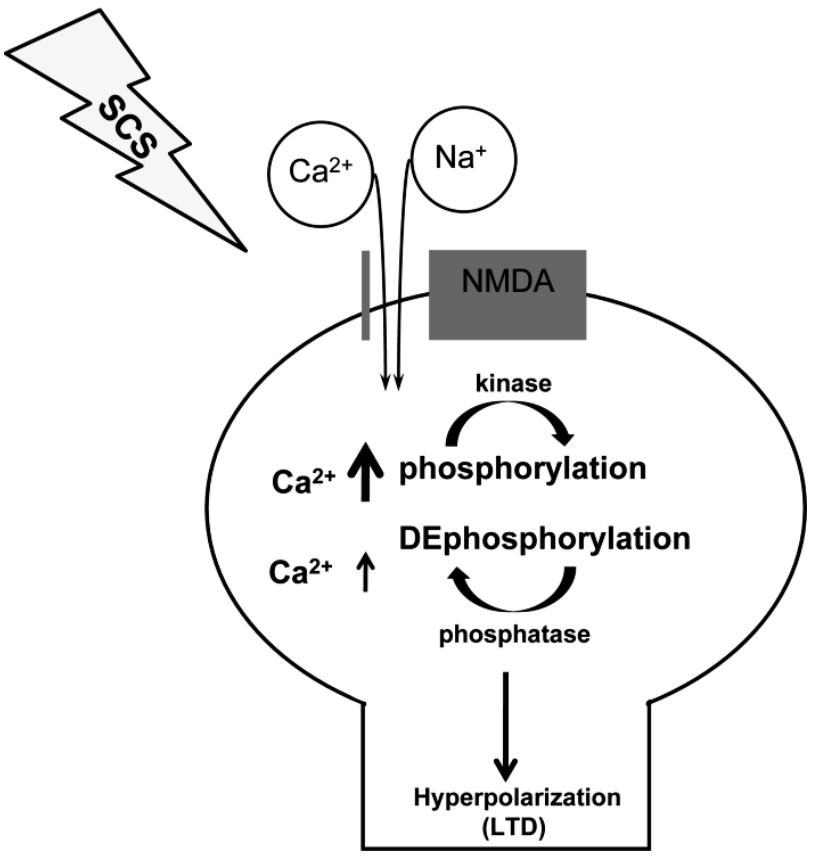

Figure 7.2. Calcium-dependent activity of the kinase/phosphatase network. SCS induces a decreased concentrations of $\mathrm{Ca}^{2+}$. This low concentration of $\mathrm{Ca}^{2+}$ activates protein phosphatases which dephosphorylates the NMDA receptor, resulting in Long Term Depotentiotion (LTD).

In the spinal cord of naive animals, LTP or central sensitization is typically induced by high frequency stimulation of C-fibers. Direct electrical stimulation of A $\delta$-fibers at low frequencies $(1 \mathrm{~Hz})$ have been reported to induce spinal LTD in non-neuropathic rats [176] [118]. Central sensitization as a result from damage to a peripheral nerve is thought as the spinal equivalent of LTP $[81,175]$, resulting from increased intracellular $\mathrm{Ca}^{2+}$ concentrations through the NMDA receptor. The high concentration of $\mathrm{Ca}^{2+}$ activates phosphokinases which in their turn activate NMDA and AMPA receptors owing to the central sensitization. In rats with neuropathic pain, low frequency $(2 \mathrm{~Hz}$ ) electroacupuncture (EA) at C-fiber strength induced LTD [239]. However high frequency $(100 \mathrm{~Hz})$ EA in the neuropathic animals induced LTP, where in naive animals EA at $100 \mathrm{~Hz}$ resulted in LTD. From this, it can be concluded that LTP and LTD depend on the frequency, type of fibers that are being stimulated and whether or not central 
sensitization is present. With respect to SCS, low frequency (4 and $60 \mathrm{~Hz}$ ) multipolar SCS in the SNI neuropathic pain model in rats resulted in better pain relief than high frequency (100 and $250 \mathrm{~Hz}$ ) SCS [121], possibly inducing LTD of the spinal cord neurons. There is however a discrepancy between EA and SCS. EA activates $C$-fibers, where SCS is thought to activate $A \beta$-fibers in the dorsal column $[75,135]$. It can however not be excluded that $\mathrm{C}$ - or $\mathrm{A} \delta$-fibers are being indirectly activated by SCS and therefore LTD may indirectly occur as the result of SCS. SCS and the frequency of $50 \mathrm{~Hz}$ used in all experiments described in this thesis allows for both induction of LTP as well as LTD or depotentiating processes (Figure 7.2).

\section{The future of Spinal Cord Stimulation in neuropathic pain}

Based on the findings in this thesis and the literature on spinal cord stimulation in neuropathic pain we demonstrate that experimental animal research is crucial in the search for improved strategies on the pain relieving effect of SCS. Although results from animal experiments do not always guaranty a successful outcome if transferred to the clinical situation, they still provide valuable information. These experimental models can be safely used to test different drugs and SCS paradigms for instance. The question remains what the "best" strategy is to reach maximal success of spinal cord stimulation. To our opinion, there is no "best" strategy, but combining findings from experimental work could proof useful. For instance, early intervention probably will improve the efficacy of SCS, especially in combination with (early) diagnostic tools [222]. When combined with a readily available drug, like ketamine, the effect of SCS can even be improved or prolonged. Promising results were recently reported on the intravenous use of ketamine in patients with neuropathic pain [192] and combined with SCS, the results might even improve. The use of pharmacotherapy in combination with SCS appears to be promising, although the best drug of choice and the route of administration is debatable. Because neuropathic pain entails many structural and biochemical changes and many molecules are involved, it is unlikely that one drug will proof to be the "miracle" drug. Ketamine, baclofen, pregabalin, clonidine and serotonin all proved to enhance the effect of SCS, and from this it is suggested that these drugs can be used in combination or in sequence, when the effect of one drug is fading.

Furthermore, inducing LTD by finding the optimal SCS frequency in patients might eventually lead to the improved and prolonged pain reducing effect of SCS on neuropathic pain. 

Bibliography 

[1] Alexander GM, van Rijn MA, van Hilten JJ, Perreault MJ, Schwartzman RJ. Changes in cerebrospinal fluid levels of pro-inflammatory cytokines in CRPS. Pain 2005;116(3):213-219.

[2] Amargos-Bosch M, Lopez-Gil X, Artigas F, Adell A. Clozapine and olanzapine, but not haloperidol, suppress serotonin efflux in the medial prefrontal cortex elicited by phencyclidine and ketamine. Int J Neuropsychopharmacol 2006;9(5):565-573.

[3] Angeby-Moller K, Berge OG, Hamers FP. Using the CatWalk method to assess weight-bearing and pain behaviour in walking rats with ankle joint monoarthritis induced by carrageenan: effects of morphine and rofecoxib. J Neurosci Methods 2008;174(1):1-9.

[4] Backonja MM. Anticonvulsants (antineuropathics) for neuropathic pain syndromes. Clin J Pain 2000;16(2 Suppl):S67-72.

[5] Backonja MM, Serra J. Pharmacologic management part 2: lesser-studied neuropathic pain diseases. Pain Med 2004;5 Suppl 1:S48-59.

[6] Backonja MM, Stacey B. Neuropathic pain symptoms relative to overall pain rating. J Pain 2004;5(9):491-497.

[7] Bardoni R, Torsney C, Tong CK, Prandini M, MacDermott AB. Presynaptic NMDA receptors modulate glutamate release from primary sensory neurons in rat spinal cord dorsal horn. J Neurosci 2004;24(11):2774-2781.

[8] Basbaum Al, Gautron M, Jazat F, Mayes M, Guilbaud G. The spectrum of fiber loss in a model of neuropathic pain in the rat: an electron microscopic study. Pain 1991;47(3):359-367.

[9] Bennett GJ. An animal model of neuropathic pain: a review. Muscle \& nerve 1993;16(10):1040-1048.

[10] Bennett GJ, Xie YK. A peripheral mononeuropathy in rat that produces disorders of pain sensation like those seen in man. Pain 1988;33(1):87-107.

[11] Berta T, Poirot O, Pertin M, Ji RR, Kellenberger S, Decosterd I. Transcriptional and functional profiles of voltage-gated $\mathrm{Na}(+)$ channels in injured and non-injured DRG neurons in the SNI model of neuropathic pain. Mol Cell Neurosci 2008;37(2):196208. 
[12] Bessou P, Perl ER. Response of cutaneous sensory units with unmyelinated fibers to noxious stimuli. J Neurophysiol 1969;32(6):1025-1043.

[13] Black JA, Cummins TR, Plumpton C, Chen YH, Hormuzdiar W, Clare JJ, Waxman SG. Upregulation of a silent sodium channel after peripheral, but not central, nerve injury in DRG neurons. J Neurophysiol 1999;82(5):2776-2785.

[14] Bliss TV, Collingridge GL. A synaptic model of memory: long-term potentiation in the hippocampus. Nature 1993;361(6407):31-39.

[15] Blonk MI, Koder BG, van den Bemt PM, Huygen FJ. Use of oral ketamine in chronic pain management: a review. Eur J Pain 2010;14(5):466-472.

[16] Brickley SG, Cull-Candy SG, Farrant M. Single-channel properties of synaptic and extrasynaptic GABAA receptors suggest differential targeting of receptor subtypes. J Neurosci 1999;19(8):2960-2973.

[17] Brock-Utne JG, Mankowitz E, Kallichurum S, Downing JW. Effects of intrathecal saline and ketamine with and without preservative on the spinal nerve roots of monkeys. S Afr Med J 1982;61(10):360-361.

[18] Bryson HM, Wilde MI. Amitriptyline. A review of its pharmacological properties and therapeutic use in chronic pain states. Drugs Aging 1996;8(6):459-476.

[19] Buckenmaier CC, McKnight GM, Winkley JV, Bleckner LL, Shannon C, Klein SM, Lyons RC, Chiles JH. Continuous peripheral nerve block for battlefield anesthesia and evacuation. Reg Anesth Pain Med 2005;30(2):202-205.

[20] Burgess SE, Gardell LR, Ossipov MH, Malan TP, Jr., Vanderah TW, Lai J, Porreca F. Time-dependent descending facilitation from the rostral ventromedial medulla maintains, but does not initiate, neuropathic pain. J Neurosci 2002;22(12):51295136.

[21] Castellani GC, Quinlan EM, Bersani F, Cooper LN, Shouval HZ. A model of bidirectional synaptic plasticity: from signaling network to channel conductance. Learn Mem 2005;12(4):423-432.

[22] Castro-Lopes JM, Coimbra A, Grant G, Arvidsson J. Ultrastructural changes of the central scalloped (C1) primary afferent endings of synaptic glomeruli in the substantia gelatinosa Rolandi of the rat after peripheral neurotomy. J Neurocytol 1990;19(3):329-337. 
[23] Cepeda MS, Lau J, Carr DB. Defining the therapeutic role of local anesthetic sympathetic blockade in complex regional pain syndrome: a narrative and systematic review. Clin J Pain 2002;18(4):216-233.

[24] Chaplan SR, Bach FW, Pogrel JW, Chung JM, Yaksh TL. Quantitative assessment of tactile allodynia in the rat paw. J Neurosci Methods 1994;53(1):55-63.

[25] Chen PE, Wyllie DJ. Pharmacological insights obtained from structure-function studies of ionotropic glutamate receptors. Br J Pharmacol 2006;147(8):839-853.

[26] Clatworthy AL, Illich PA, Castro GA, Walters ET. Role of peri-axonal inflammation in the development of thermal hyperalgesia and guarding behavior in a rat model of neuropathic pain. Neurosci Lett 1995;184(1):5-8.

[27] Coderre TJ, Kumar N, Lefebvre CD, Yu JS. Evidence that gabapentin reduces neuropathic pain by inhibiting the spinal release of glutamate. J Neurochem 2005;94(4):1131-1139.

[28] Coderre TJ, Xanthos DN, Francis L, Bennett GJ. Chronic post-ischemia pain (CPIP): a novel animal model of complex regional pain syndrome-type I (CRPS-I; reflex sympathetic dystrophy) produced by prolonged hindpaw ischemia and reperfusion in the rat. Pain 2004;112(1-2):94-105.

[29] Coggeshall RE, Dougherty PM, Pover CM, Carlton SM. Is large myelinated fiber loss associated with hyperalgesia in a model of experimental peripheral neuropathy in the rat? Pain 1993;52(2):233-242.

[30] Correll GE, Maleki J, Gracely EJ, Muir JJ, Harbut RE. Subanesthetic ketamine infusion therapy: a retrospective analysis of a novel therapeutic approach to complex regional pain syndrome. Pain Med 2004;5(3):263-275.

[31] Crisp T, Perrotti JM, Smith DL, Stafinsky JL, Smith DJ. The local monoaminergic dependency of spinal ketamine. Eur J Pharmacol 1991;194(2-3):167-172.

[32] Cui JG, Holmin S, Mathiesen T, Meyerson BA, Linderoth B. Possible role of inflammatory mediators in tactile hypersensitivity in rat models of mononeuropathy. Pain 2000;88(3):239-248.

[33] Cui JG, Linderoth B, Meyerson BA. Effects of spinal cord stimulation on touchevoked allodynia involve GABAergic mechanisms. An experimental study in the mononeuropathic rat. Pain 1996;66(2-3):287-295. 
[34] Cui JG, Meyerson BA, Linderoth B. Opposite effects of spinal cord stimulation in different phases of carrageenan-induced hyperalgesia. Eur J Pain 1999;3(4):365-374.

[35] Cui JG, Meyerson BA, Sollevi A, Linderoth B. Effect of spinal cord stimulation on tactile hypersensitivity in mononeuropathic rats is potentiated by simultaneous GABA(B) and adenosine receptor activation. Neurosci Lett 1998;247(2-3):183-186.

[36] Cui JG, O'Connor WT, Ungerstedt U, Linderoth B, Meyerson BA. Spinal cord stimulation attenuates augmented dorsal horn release of excitatory amino acids in mononeuropathy via a GABAergic mechanism. Pain 1997;73(1):87-95.

[37] Cutler G, Marshall LA, Chin N, Baribault H, Kassner PD. Significant gene content variation characterizes the genomes of inbred mouse strains. Genome Res 2007; 17(12):1743-1754.

[38] Cvrcek P. Side effects of ketamine in the long-term treatment of neuropathic pain. Pain Med 2008;9(2):253-257.

[39] de Mos M, de Bruijn AG, Huygen FJ, Dieleman JP, Stricker BH, Sturkenboom MC. The incidence of complex regional pain syndrome: a population-based study. Pain 2007;129(1-2):12-20.

[40] de Rooij AM, Florencia Gosso M, Haasnoot GW, Marinus J, Verduijn W, Claas FH, van den Maagdenberg AM, van Hilten JJ. HLA-B62 and HLA-DQ8 are associated with Complex Regional Pain Syndrome with fixed dystonia. Pain 2009;145(1-2):8285.

[41] Decosterd I, Woolf CJ. Spared nerve injury: an animal model of persistent peripheral neuropathic pain. Pain 2000;87(2):149-158.

[42] DeLeo JA, Rutkowski MD. Gender differences in rat neuropathic pain sensitivity is dependent on strain. Neurosci Lett 2000;282(3):197-199.

[43] Deumens R, Jaken RJ, Marcus MA, Joosten EA. The CatWalk gait analysis in assessment of both dynamic and static gait changes after adult rat sciatic nerve resection. J Neurosci Methods 2007;164(1):120-130.

[44] Dib-Hajj SD, Binshtok AM, Cummins TR, Jarvis MF, Samad T, Zimmermann K. Voltage-gated sodium channels in pain states: role in pathophysiology and targets for treatment. Brain Res Rev 2009;60(1):65-83. 
[45] Dickenson AH, Chapman V, Green GM. The pharmacology of excitatory and inhibitory amino acid-mediated events in the transmission and modulation of pain in the spinal cord. Gen Pharmacol 1997;28(5):633-638.

[46] Dominguez CA, Lidman O, Hao JX, Diez M, Tuncel J, Olsson T, Wiesenfeld-Hallin Z, Piehl F, Xu XJ. Genetic analysis of neuropathic pain-like behavior following peripheral nerve injury suggests a role of the major histocompatibility complex in development of allodynia. Pain 2008;136(3):313-319.

[47] Eaton MJ, Martinez MA, Karmally S. A single intrathecal injection of GABA permanently reverses neuropathic pain after nerve injury. Brain Res 1999;835(2):334-339.

[48] Eaton MJ, Plunkett JA, Karmally S, Martinez MA, Montanez K. Changes in GADand GABA- immunoreactivity in the spinal dorsal horn after peripheral nerve injury and promotion of recovery by lumbar transplant of immortalized serotonergic precursors. J Chem Neuroanat 1998;16(1):57-72.

[49] Eide PK, Stubhaug A, Breivik H, Oye I. Reply to S.T. Meller: Ketamine: relief from chronic pain through actions at the NMDA receptor. Pain 1997;72(1-2):289-291.

[50] Eisenach JC, Yaksh TL. Epidural ketamine in healthy children--what's the point? Anesth Analg 2003;96(2):626; author reply 626-627.

[51] Eisenberg E, Erlich T, Zinder O, Lichinsky S, Diamond E, Pud D, Davar G. Plasma endothelin-1 levels in patients with complex regional pain syndrome. Eur J Pain 2004;8(6):533-538.

[52] Ekberg J, Jayamanne A, Vaughan CW, Aslan S, Thomas L, Mould J, Drinkwater R, Baker MD, Abrahamsen B, Wood JN, Adams DJ, Christie MJ, Lewis RJ. muOconotoxin MrVIB selectively blocks Nav1.8 sensory neuron specific sodium channels and chronic pain behavior without motor deficits. Proc Natl Acad Sci U S A 2006;103(45):17030-17035.

[53] El-Khoury C, Hawwa N, Baliki M, Atweh SF, Jabbur SJ, Saade NE. Attenuation of neuropathic pain by segmental and supraspinal activation of the dorsal column system in awake rats. Neuroscience 2002;112(3):541-553.

[54] Eliav E, Herzberg U, Ruda MA, Bennett GJ. Neuropathic pain from an experimental neuritis of the rat sciatic nerve. Pain 1999;83(2):169-182. 
[55] Felsby S, Nielsen J, Arendt-Nielsen L, Jensen TS. NMDA receptor blockade in chronic neuropathic pain: a comparison of ketamine and magnesium chloride. Pain $1996 ; 64(2): 283-291$

[56] Finch PM, Knudsen L, Drummond PD. Reduction of allodynia in patients with complex regional pain syndrome: A double-blind placebo-controlled trial of topical ketamine. Pain 2009;146(1-2):18-25.

[57] Finnerup NB, Otto M, Jensen TS, Sindrup SH. An evidence-based algorithm for the treatment of neuropathic pain. MedGenMed 2007;9(2):36.

[58] Gabriel AF, Marcus MA, Honig WM, Walenkamp GH, Joosten EA. The CatWalk method: a detailed analysis of behavioral changes after acute inflammatory pain in the rat. J Neurosci Methods 2007;163(1):9-16.

[59] Gabriel AF, Marcus MA, Walenkamp GH, Joosten EA. The CatWalk method: assessment of mechanical allodynia in experimental chronic pain. Behav Brain Res 2009;198(2):477-480.

[60] Gao X, Kim HK, Chung JM, Chung K. Enhancement of NMDA receptor phosphorylation of the spinal dorsal horn and nucleus gracilis neurons in neuropathic rats. Pain 2005;116(1-2):62-72.

[61] Gensel JC, Tovar CA, Hamers FP, Deibert RJ, Beattie MS, Bresnahan JC. Behavioral and histological characterization of unilateral cervical spinal cord contusion injury in rats. J Neurotrauma 2006;23(1):36-54.

[62] Gosso MF, de Rooij AM, Alsina-Sanchis E, Kamphorst JT, Marinus J, van Hilten JJ, van den Maagdenberg AM. Systematic mutation analysis of seven dystonia genes in complex regional pain syndrome with fixed dystonia. J Neurol 2010;257(5):820824.

[63] Groeneweg JG, Huygen FJ, Heijmans-Antonissen C, Niehof S, Zijlstra FJ. Increased endothelin-1 and diminished nitric oxide levels in blister fluids of patients with intermediate cold type complex regional pain syndrome type 1. BMC Musculoskelet Disord 2006;7:91.

[64] Guo TZ, Offley SC, Boyd EA, Jacobs CR, Kingery WS. Substance P signaling contributes to the vascular and nociceptive abnormalities observed in a tibial 
fracture rat model of complex regional pain syndrome type I. Pain 2004;108(12):95-107.

[65] Guo W, Zou S, Guan Y, Ikeda T, Tal M, Dubner R, Ren K. Tyrosine phosphorylation of the NR2B subunit of the NMDA receptor in the spinal cord during the development and maintenance of inflammatory hyperalgesia. J Neurosci 2002;22(14):6208-6217.

[66] Hamers FP, Lankhorst AJ, van Laar TJ, Veldhuis WB, Gispen WH. Automated quantitative gait analysis during overground locomotion in the rat: its application to spinal cord contusion and transection injuries. J Neurotrauma 2001;18(2):187-201.

[67] Handwerker HO, Kobal G. Psychophysiology of experimentally induced pain. Physiol Rev 1993;73(3):639-671.

[68] Harden RN, Bruehl S, Perez RS, Birklein F, Marinus J, Maihofner C, Lubenow T, Buvanendran A, Mackey S, Graciosa J, Mogilevski M, Ramsden C, Chont M, Vatine JJ. Validation of proposed diagnostic criteria (the "Budapest Criteria") for Complex Regional Pain Syndrome. Pain 2010;150(2):268-274.

[69] Harden RN, Bruehl S, Stanton-Hicks M, Wilson PR. Proposed new diagnostic criteria for complex regional pain syndrome. Pain Med 2007;8(4):326-331.

[70] Harke H, Gretenkort P, Ladleif HU, Rahman S. Spinal cord stimulation in sympathetically maintained complex regional pain syndrome type I with severe disability. A prospective clinical study. Eur J Pain 2005;9(4):363-373.

[71] Harney D, Magner JJ, O'Keeffe D. Early intervention with spinal cord stimulation in the management of a chronic regional pain syndrome. Ir Med J 2005;98(3):89-90.

[72] He XH, Zang Y, Chen X, Pang RP, Xu JT, Zhou X, Wei XH, Li YY, Xin WJ, Qin ZH, Liu XG. TNF-alpha contributes to up-regulation of Nav1.3 and Nav1.8 in DRG neurons following motor fiber injury. Pain 2010.

[73] Herman RM, D'Luzansky SC, Ippolito R. Intrathecal baclofen suppresses central pain in patients with spinal lesions. A pilot study. Clin J Pain 1992;8(4):338-345.

[74] Hocking G, Cousins MJ. Ketamine in chronic pain management: an evidence-based review. Anesth Analg 2003;97(6):1730-1739.

[75] Holsheimer J. Which neuronal elements are activated directly by spinal cord stimulation. Neuromodulation 2002;5(1):25-31. 
[76] Holsheimer J. Principles of neurostimulation, Vol. 15. Cardiff: Elsevier, 2003.

[77] Huygen FJ, De Bruijn AG, De Bruin MT, Groeneweg JG, Klein J, Zijlstra FJ. Evidence for local inflammation in complex regional pain syndrome type 1. Mediators Inflamm 2002;11(1):47-51.

[78] Ibuki T, Hama AT, Wang XT, Pappas GD, Sagen J. Loss of GABA-immunoreactivity in the spinal dorsal horn of rats with peripheral nerve injury and promotion of recovery by adrenal medullary grafts. Neuroscience 1997;76(3):845-858.

[79] Iwamoto ET, Marion L. Adrenergic, serotonergic and cholinergic components of nicotinic antinociception in rats. J Pharmacol Exp Ther 1993;265(2):777-789.

[80] Iwata H, Takasusuki T, Yamaguchi S, Hori Y. NMDA receptor 2B subunit-mediated synaptic transmission in the superficial dorsal horn of peripheral nerve-injured neuropathic mice. Brain Res 2007;1135(1):92-101.

[81] Ji RR, Kohno T, Moore KA, Woolf CJ. Central sensitization and LTP: do pain and memory share similar mechanisms? Trends Neurosci 2003;26(12):696-705.

[82] Ji RR, Woolf CJ. Neuronal plasticity and signal transduction in nociceptive neurons: implications for the initiation and maintenance of pathological pain. Neurobiol Dis $2001 ; 8(1): 1-10$.

[83] Jin SX, Zhuang ZY, Woolf CJ, Ji RR. p38 mitogen-activated protein kinase is activated after a spinal nerve ligation in spinal cord microglia and dorsal root ganglion neurons and contributes to the generation of neuropathic pain. J Neurosci 2003;23(10):4017-4022.

[84] Kangrga I, Jiang MC, Randic M. Actions of (-)-baclofen on rat dorsal horn neurons. Brain Res 1991;562(2):265-275.

[85] Kemler MA, Barendse GA, van Kleef M, de Vet HC, Rijks CP, Furnee CA, van den Wildenberg FA. Spinal cord stimulation in patients with chronic reflex sympathetic dystrophy. N Engl J Med 2000;343(9):618-624.

[86] Kemler MA, De Vet HC, Barendse GA, Van Den Wildenberg FA, Van Kleef M. The effect of spinal cord stimulation in patients with chronic reflex sympathetic dystrophy: two years' follow-up of the randomized controlled trial. Ann Neurol 2004;55(1):13-18. 
[87] Kemler MA, de Vet HC, Barendse GA, van den Wildenberg FA, van Kleef M. Spinal cord stimulation for chronic reflex sympathetic dystrophy--five-year follow-up. $\mathrm{N}$ Engl J Med 2006;354(22):2394-2396.

[88] Kemler MA, de Vet HC, Barendse GA, van den Wildenberg FA, van Kleef M. Effect of spinal cord stimulation for chronic complex regional pain syndrome Type I: fiveyear final follow-up of patients in a randomized controlled trial. J Neurosurg 2008;108(2):292-298.

[89] Kemler MA, van de Vusse AC, van den Berg-Loonen EM, Barendse GA, van Kleef M, Weber WE. HLA-DQ1 associated with reflex sympathetic dystrophy. Neurology 1999;53(6):1350-1351.

[90] Kiefer RT, Rohr P, Ploppa A, Dieterich HJ, Grothusen J, Koffler S, Altemeyer KH, Unertl K, Schwartzman RJ. Efficacy of ketamine in anesthetic dosage for the treatment of refractory complex regional pain syndrome: an open-label phase II study. Pain Med 2008;9(8):1173-1201.

[91] Kim CH, Oh Y, Chung JM, Chung K. The changes in expression of three subtypes of TTX sensitive sodium channels in sensory neurons after spinal nerve ligation. Brain Res Mol Brain Res 2001;95(1-2):153-161.

[92] Kim SH, Chung JM. An experimental model for peripheral neuropathy produced by segmental spinal nerve ligation in the rat. Pain 1992;50(3):355-363.

[93] Kingery WS, Davies MF, Clark JD. A substance P receptor (NK1) antagonist can reverse vascular and nociceptive abnormalities in a rat model of complex regional pain syndrome type II. Pain 2003;104(1-2):75-84.

[94] Kleinschnitz C, Hofstetter HH, Meuth SG, Braeuninger S, Sommer C, Stoll G. T cell infiltration after chronic constriction injury of mouse sciatic nerve is associated with interleukin-17 expression. Exp Neurol 2006;200(2):480-485.

[95] Klusakova I, Dubovy P. Experimental models of peripheral neuropathic pain based on traumatic nerve injuries - an anatomical perspective. Ann Anat 2009;191(3):248259.

[96] Koopmans GC, Deumens R, Honig WM, Hamers FP, Steinbusch HW, Joosten EA. The assessment of locomotor function in spinal cord injured rats: the importance of objective analysis of coordination. J Neurotrauma 2005;22(2):214-225. 
[97] Krames E. Spinal Cord Stimulation: Indications, Mechanism of Action, and Efficacy. Curr Rev Pain 1999;3(6):419-426.

[98] Kumar K, Hunter G, Demeria D. Spinal cord stimulation in treatment of chronic benign pain: challenges in treatment planning and present status, a 22-year experience. Neurosurgery 2006;58(3):481-496; discussion 481-496.

[99] Kumar K, Taylor RS, Jacques L, Eldabe S, Meglio M, Molet J, Thomson S, O'Callaghan J, Eisenberg E, Milbouw G, Buchser E, Fortini G, Richardson J, North RB. Spinal cord stimulation versus conventional medical management for neuropathic pain: a multicentre randomised controlled trial in patients with failed back surgery syndrome. Pain 2007;132(1-2):179-188.

[100] Kumar K, Taylor RS, Jacques L, Eldabe S, Meglio M, Molet J, Thomson S, O'Callaghan J, Eisenberg E, Milbouw G, Buchser E, Fortini G, Richardson J, North RB. The effects of spinal cord stimulation in neuropathic pain are sustained: a 24month follow-up of the prospective randomized controlled multicenter trial of the effectiveness of spinal cord stimulation. Neurosurgery 2008;63(4):762-770; discussion 770 .

[101] Kumar K, Toth C, Nath RK, Laing P. Epidural spinal cord stimulation for treatment of chronic pain--some predictors of success. A 15-year experience. Surg Neurol 1998;50(2):110-120; discussion 120-111.

[102] LaCroix-Fralish ML, Rutkowski MD, Weinstein JN, Mogil JS, Deleo JA. The magnitude of mechanical allodynia in a rodent model of lumbar radiculopathy is dependent on strain and sex. Spine (Phila Pa 1976) 2005;30(16):1821-1827.

[103] Lai J, Gold MS, Kim CS, Bian D, Ossipov MH, Hunter JC, Porreca F. Inhibition of neuropathic pain by decreased expression of the tetrodotoxin-resistant sodium channel, NaV1.8. Pain 2002;95(1-2):143-152.

[104] Lante F, Cavalier M, Cohen-Solal C, Guiramand J, Vignes M. Developmental switch from LTD to LTP in low frequency-induced plasticity. Hippocampus 2006;16(11):981-989.

[105] Latremoliere A, Woolf CJ. Central sensitization: a generator of pain hypersensitivity by central neural plasticity. J Pain 2009;10(9):895-926. 
[106] Li D, Yang H, Meyerson BA, Linderoth B. Response to spinal cord stimulation in variants of the spared nerve injury pain model. Neurosci Lett 2006;400(1-2):115120.

[107] Li L, Xian CJ, Zhong JH, Zhou XF. Effect of lumbar 5 ventral root transection on pain behaviors: a novel rat model for neuropathic pain without axotomy of primary sensory neurons. Exp Neurol 2002;175(1):23-34.

[108] Li Y, Dorsi MJ, Meyer RA, Belzberg AJ. Mechanical hyperalgesia after an L5 spinal nerve lesion in the rat is not dependent on input from injured nerve fibers. Pain 2000;85(3):493-502.

[109] Lind G, Meyerson BA, Winter J, Linderoth B. Intrathecal baclofen as adjuvant therapy to enhance the effect of spinal cord stimulation in neuropathic pain: a pilot study. Eur J Pain 2004;8(4):377-383.

[110] Lindefors N, Barati S, O'Connor WT. Differential effects of single and repeated ketamine administration on dopamine, serotonin and GABA transmission in rat medial prefrontal cortex. Brain Res 1997;759(2):205-212.

[111] Linden DJ. Long-term synaptic depression in the mammalian brain. Neuron 1994;12(3):457-472.

[112] Linderoth B, Stiller CO, Gunasekera L, O'Connor WT, Franck J, Gazelius B, Brodin E. Release of neurotransmitters in the CNS by spinal cord stimulation: survey of present state of knowledge and recent experimental studies. Stereotact Funct Neurosurg 1993;61(4):157-170.

[113] Linderoth B, Stiller CO, Gunasekera L, O'Connor WT, Ungerstedt U, Brodin E. Gamma-aminobutyric acid is released in the dorsal horn by electrical spinal cord stimulation: an in vivo microdialysis study in the rat. Neurosurgery 1994;34(3):484488; discussion 488-489.

[114] Lindia JA, Kohler MG, Martin WJ, Abbadie C. Relationship between sodium channel NaV1.3 expression and neuropathic pain behavior in rats. Pain 2005;117(1-2):145-153.

[115] Lisman J. A mechanism for the Hebb and the anti-Hebb processes underlying learning and memory. Proc Natl Acad Sci U S A 1989;86(23):9574-9578. 
[116] Liu H, Wang H, Sheng M, Jan LY, Jan YN, Basbaum Al. Evidence for presynaptic Nmethyl-D-aspartate autoreceptors in the spinal cord dorsal horn. Proc Natl Acad Sci U S A 1994;91(18):8383-8387.

[117] Liu T, van Rooijen N, Tracey DJ. Depletion of macrophages reduces axonal degeneration and hyperalgesia following nerve injury. Pain 2000;86(1-2):25-32.

[118] Liu XG, Morton CR, Azkue JJ, Zimmermann M, Sandkuhler J. Long-term depression of C-fibre-evoked spinal field potentials by stimulation of primary afferent A deltafibres in the adult rat. Eur J Neurosci 1998;10(10):3069-3075.

[119] Lunn ER, Perry VH, Brown MC, Rosen H, Gordon S. Absence of Wallerian Degeneration does not Hinder Regeneration in Peripheral Nerve. Eur J Neurosci 1989;1(1):27-33.

[120] Lyon MF, Ogunkolade BW, Brown MC, Atherton DJ, Perry VH. A gene affecting Wallerian nerve degeneration maps distally on mouse chromosome 4. Proc Natl Acad Sci U S A 1993;90(20):9717-9720.

[121] Maeda Y, Wacnik PW, Sluka KA. Low frequencies, but not high frequencies of bipolar spinal cord stimulation reduce cutaneous and muscle hyperalgesia induced by nerve injury. Pain 2008;138(1):143-152.

[122] Mailis A, Wade J. Profile of Caucasian women with possible genetic predisposition to reflex sympathetic dystrophy: a pilot study. Clin J Pain 1994;10(3):210-217.

[123] Malinovsky JM, Lepage JY, Cozian A, Mussini JM, Pinaudt M, Souron R. Is ketamine or its preservative responsible for neurotoxicity in the rabbit? Anesthesiology 1993;78(1):109-115.

[124] Malkmus SA, Yaksh TL. Intrathecal catheterization and drug delivery in the rat. Methods Mol Med 2004;99:109-121.

[125] Malmberg AB, Basbaum Al. Partial sciatic nerve injury in the mouse as a model of neuropathic pain: behavioral and neuroanatomical correlates. Pain 1998;76(12):215-222.

[126] Mao J, Price DD, Hayes RL, Lu J, Mayer DJ, Frenk H. Intrathecal treatment with dextrorphan or ketamine potently reduces pain-related behaviors in a rat model of peripheral mononeuropathy. Brain Res 1993;605(1):164-168. 
[127] Marchand F, Bischop T, Anand R, Grist J, Clark AK, McMahon SB. The CatWalk method: Behavioral changes and effect on analgesic drugs in inflammatory and neuropathic pain models. Soc Neurosci Abstr 2007;181(19).

[128] Mayer ML, Westbrook GL, Guthrie PB. Voltage-dependent block by Mg2+ of NMDA responses in spinal cord neurones. Nature 1984;309(5965):261-263.

[129] Mei X, Wang W, Li Y, Zhang H, Wu S, Xu L. Inhibiting astrocytic activation: a novel analgesic mechanism of ketamine at the spinal level? J Neurochem 2009;109(6):1691-1700.

[130] Meller ST. Ketamine: relief from chronic pain through actions at the NMDA receptor? Pain 1996;68(2-3):435-436.

[131] Melzack R, Wall PD. Pain mechanisms: a new theory. Science 1965;150(699):971979.

[132] Merskey H, Bogduk N. 1994. Task Force on Taxonomyof the International Association for the Study of Pain: Classification of Chronic Pain. Description of Pain Syndromes and Definitions of Pain Terms. IASP Press, Seattle, WA.

[133] Merskey H, Bogduk N. Classification of chronic pain: descriptions of chronic pain syndromes and defenitions of pain terms. Seattle: IASP Press:. 1994:41-42.

[134] Meyerson BA, Herregodts P, Linderoth B, Ren B. An experimental animal model of spinal cord stimulation for pain. Stereotactic and functional neurosurgery 1994;62(1-4):256-262.

[135] Meyerson BA, Ren B, Herregodts P, Linderoth B. Spinal cord stimulation in animal models of mononeuropathy: effects on the withdrawal response and the flexor reflex. Pain 1995;61(2):229-243.

[136] Michaelis M, Liu X, Janig W. Axotomized and intact muscle afferents but no skin afferents develop ongoing discharges of dorsal root ganglion origin after peripheral nerve lesion. J Neurosci 2000;20(7):2742-2748.

[137] Millan MJ. The induction of pain: an integrative review. Prog Neurobiol 1999;57(1):1-164.

[138] Millecamps M, Laferriere A, Ragavendran JV, Stone LS, Coderre TJ. Role of peripheral endothelin receptors in an animal model of complex regional pain syndrome type 1 (CRPS-I). Pain 2010;151(1):174-183. 
[139] Mintz IM, Bean BP. GABAB receptor inhibition of P-type Ca2+ channels in central neurons. Neuron 1993;10(5):889-898.

[140] Mitchell JJ, Anderson KJ. Quantitative autoradiographic analysis of excitatory amino acid receptors in the cat spinal cord. Neurosci Lett 1991;124(2):269-272.

[141] Mogil JS. Animal models of pain: progress and challenges. Nat Rev Neurosci 2009;10(4):283-294

[142] Mogil JS, Crager SE. What should we be measuring in behavioral studies of chronic pain in animals? Pain 2004;112(1-2):12-15

[143] Mogil JS, Graham AC, Ritchie J, Hughes SF, Austin JS, Schorscher-Petcu A, Langford DJ, Bennett GJ. Hypolocomotion, asymmetrically directed behaviors (licking, lifting, flinching, and shaking) and dynamic weight bearing (gait) changes are not measures of neuropathic pain in mice. Mol Pain 2010;6:34.

[144] Mogil JS, Wilson SG, Bon K, Lee SE, Chung K, Raber P, Pieper JO, Hain HS, Belknap JK, Hubert L, Elmer GI, Chung JM, Devor M. Heritability of nociception I: responses of 11 inbred mouse strains on 12 measures of nociception. Pain 1999;80(1-2):67-82.

[145] Mogil JS, Wilson SG, Bon K, Lee SE, Chung K, Raber P, Pieper JO, Hain HS, Belknap JK, Hubert L, Elmer GI, Chung JM, Devor M. Heritability of nociception II. 'Types' of nociception revealed by genetic correlation analysis. Pain 1999;80(12):83-93.

[146] Molander C, Xu Q, Grant G. The cytoarchitectonic organization of the spinal cord in the rat. I. The lower thoracic and lumbosacral cord. J Comp Neurol 1984;230(1):133-141.

[147] Moore KA, Kohno T, Karchewski LA, Scholz J, Baba H, Woolf CJ. Partial peripheral nerve injury promotes a selective loss of GABAergic inhibition in the superficial dorsal horn of the spinal cord. J Neurosci 2002;22(15):6724-6731.

[148] Morin N, Owolabi SA, Harty MW, Papa EF, Tracy TF, Jr., Shaw SK, Kim M, Saab CY. Neutrophils invade lumbar dorsal root ganglia after chronic constriction injury of the sciatic nerve. J Neuroimmunol 2007;184(1-2):164-171. 
[149] Myers RR, Heckman HM, Rodriguez M. Reduced hyperalgesia in nerve-injured WLD mice: relationship to nerve fiber phagocytosis, axonal degeneration, and regeneration in normal mice. Exp Neurol 1996;141(1):94-101.

[150] Nassar MA, Baker MD, Levato A, Ingram R, Mallucci G, McMahon SB, Wood JN. Nerve injury induces robust allodynia and ectopic discharges in Nav1.3 null mutant mice. Mol Pain 2006;2:33.

[151] Nassar MA, Levato A, Stirling LC, Wood JN. Neuropathic pain develops normally in mice lacking both $\mathrm{Na}(\mathrm{v}) 1.7$ and $\mathrm{Na}(\mathrm{v}) 1.8$. Mol Pain 2005;1:24.

[152] Neugebauer V, Lucke T, Grubb B, Schaible HG. The involvement of N-methyl-Daspartate (NMDA) and non-NMDA receptors in the responsiveness of rat spinal neurons with input from the chronically inflamed ankle. Neurosci Lett 1994;170(2):237-240.

[153] Neveu D, Zucker RS. Postsynaptic levels of [Ca2+]i needed to trigger LTD and LTP. Neuron 1996;16(3):619-629.

[154] Nguyen D, Deng P, Matthews EA, Kim DS, Feng G, Dickenson AH, Xu ZC, Luo ZD. Enhanced pre-synaptic glutamate release in deep-dorsal horn contributes to calcium channel alpha-2-delta-1 protein-mediated spinal sensitization and behavioral hypersensitivity. Mol Pain 2009;5:6.

[155] North RB, Ewend MG, Lawton MT, Kidd DH, Piantadosi S. Failed back surgery syndrome: 5-year follow-up after spinal cord stimulator implantation. Neurosurgery 1991;28(5):692-699.

[156] North RB, Kidd DH, Farrokhi F, Piantadosi SA. Spinal cord stimulation versus repeated lumbosacral spine surgery for chronic pain: a randomized, controlled trial. Neurosurgery 2005;56(1):98-106; discussion 106-107.

[157] Okamoto K, Martin DP, Schmelzer JD, Mitsui Y, Low PA. Pro- and antiinflammatory cytokine gene expression in rat sciatic nerve chronic constriction injury model of neuropathic pain. Exp Neurol 2001;169(2):386-391.

[158] Oliver PL, Bitoun E, Davies KE. Comparative genetic analysis: the utility of mouse genetic systems for studying human monogenic disease. Mamm Genome 2007;18(6-7):412-424. 
[159] Palecek J, Dougherty PM, Kim SH, Paleckova V, Lekan H, Chung JM, Carlton SM, Willis WD. Responses of spinothalamic tract neurons to mechanical and thermal stimuli in an experimental model of peripheral neuropathy in primates. J Neurophysiol 1992;68(6):1951-1966.

[160] Perez RS, Zollinger PE, Dijkstra PU, Thomassen-Hilgersom IL, Zuurmond WW, Rosenbrand KC, Geertzen JH. Evidence based guidelines for complex regional pain syndrome type 1. BMC Neurol 2010;10:20.

[161] Persson AK, Gebauer M, Jordan S, Metz-Weidmann C, Schulte AM, Schneider HC, Ding-Pfennigdorff D, Thun J, Xu XJ, Wiesenfeld-Hallin Z, Darvasi A, Fried K, Devor M. Correlational analysis for identifying genes whose regulation contributes to chronic neuropathic pain. Mol Pain 2009;5:7.

[162] Persson AK, Thun J, Xu XJ, Wiesenfeld-Hallin Z, Strom M, Devor M, Lidman O, Fried K. Autotomy behavior correlates with the DRG and spinal expression of sodium channels in inbred mouse strains. Brain Res 2009;1285:1-13.

[163] Pertovaara A, Kontinen VK, Kalso EA. Chronic spinal nerve ligation induces changes in response characteristics of nociceptive spinal dorsal horn neurons and in their descending regulation originating in the periaqueductal gray in the rat. Exp Neurol 1997;147(2):428-436.

[164] Piesla MJ, Leventhal L, Strassle BW, Harrison JE, Cummons TA, Lu P, Whiteside GT. Abnormal gait, due to inflammation but not nerve injury, reflects enhanced nociception in preclinical pain models. Brain Res 2009;1295:89-98.

[165] Qian J, Brown SD, Carlton SM. Systemic ketamine attenuates nociceptive behaviors in a rat model of peripheral neuropathy. Brain Res 1996;715(1-2):51-62.

[166] Raja SN, Campbell JN, Meyer RA. Evidence for different mechanisms of primary and secondary hyperalgesia following heat injury to the glabrous skin. Brain 1984;107 ( Pt 4):1179-1188.

[167] Ramer MS, French GD, Bisby MA. Wallerian degeneration is required for both neuropathic pain and sympathetic sprouting into the DRG. Pain 1997;72(1-2):7178. 
[168] Rauck RL, Eisenach JC, Jackson K, Young LD, Southern J. Epidural clonidine treatment for refractory reflex sympathetic dystrophy. Anesthesiology 1993;79(6):1163-1169; discussion 1127A.

[169] Rees H, Roberts MH. Antinociceptive effects of dorsal column stimulation in the rat: involvement of the anterior pretectal nucleus. J Physiol 1989;417:375-388.

[170] Reynolds IJ, Harris KM, Miller RJ. NMDA receptor antagonists that bind to the strychnine-insensitive glycine site and inhibit NMDA-induced Ca2+ fluxes and [3H]GABA release. Eur J Pharmacol 1989;172(1):9-17.

[171] Rigaud M, Gemes G, Barabas ME, Chernoff DI, Abram SE, Stucky CL, Hogan QH. Species and strain differences in rodent sciatic nerve anatomy: implications for studies of neuropathic pain. Pain 2008;136(1-2):188-201.

[172] Rush AM, Cummins TR, Waxman SG. Multiple sodium channels and their roles in electrogenesis within dorsal root ganglion neurons. J Physiol 2007;579(Pt 1):1-14.

[173] Saade NE, Al Amin H, Chalouhi S, Baki SA, Jabbur SJ, Atweh SF. Spinal pathways involved in supraspinal modulation of neuropathic manifestations in rats. Pain 2006;126(1-3):280-293.

[174] Saade NE, Jabbur SJ. Nociceptive behavior in animal models for peripheral neuropathy: spinal and supraspinal mechanisms. Prog Neurobiol 2008;86(1):22-47.

[175] Sandkuhler J. Learning and memory in pain pathways. Pain 2000;88(2):113-118.

[176] Sandkuhler J, Chen JG, Cheng G, Randic M. Low-frequency stimulation of afferent Adelta-fibers induces long-term depression at primary afferent synapses with substantia gelatinosa neurons in the rat. J Neurosci 1997;17(16):6483-6491.

[177] Sandroni P, Benrud-Larson LM, McClelland RL, Low PA. Complex regional pain syndrome type I: incidence and prevalence in Olmsted county, a population-based study. Pain 2003;103(1-2):199-207.

[178] Saranita J, Childs D, Saranita AD. Spinal cord stimulation in the treatment of complex regional pain syndrome (CRPS) of the lower extremity: a case report. J Foot Ankle Surg 2009;48(1):52-55.

[179] Schechtmann G, Lind G, Winter J, Meyerson BA, Linderoth B. Intrathecal clonidine and baclofen enhance the pain-relieving effect of spinal cord stimulation: a 
comparative placebo-controlled, randomized trial. Neurosurgery 2010;67(1):173181.

[180] Schechtmann G, Song Z, Ultenius C, Meyerson BA, Linderoth B. Cholinergic mechanisms involved in the pain relieving effect of spinal cord stimulation in a model of neuropathy. Pain 2008;139(1):136-145.

[181] Schechtmann G, Wallin J, Meyerson BA, Linderoth B. Intrathecal clonidine potentiates suppression of tactile hypersensitivity by spinal cord stimulation in a model of neuropathy. Anesth Analg 2004;99(1):135-139.

[182] Schinkel C, Gaertner A, Zaspel J, Zedler S, Faist E, Schuermann M. Inflammatory mediators are altered in the acute phase of posttraumatic complex regional pain syndrome. Clin J Pain 2006;22(3):235-239.

[183] Schmidtko A, Luo C, Gao W, Geisslinger G, Kuner R, Tegeder I. Genetic deletion of synapsin II reduces neuropathic pain due to reduced glutamate but increased GABA in the spinal cord dorsal horn. Pain 2008.

[184] Schnoebel R, Wolff M, Peters SC, Brau ME, Scholz A, Hempelmann G, Olschewski $\mathrm{H}$, Olschewski A. Ketamine impairs excitability in superficial dorsal horn neurones by blocking sodium and voltage-gated potassium currents. $\mathrm{Br} J$ Pharmacol 2005;146(6):826-833.

[185] Schwartzman RJ, Alexander GM, Grothusen JR, Paylor T, Reichenberger E, Perreault M. Outpatient intravenous ketamine for the treatment of complex regional pain syndrome: a double-blind placebo controlled study. Pain 2009;147(1-3):107-115.

[186] Schwartzman RJ, Erwin KL, Alexander GM. The natural history of complex regional pain syndrome. Clin J Pain 2009;25(4):273-280.

[187] Seltzer Z, Dubner R, Shir Y. A novel behavioral model of neuropathic pain disorders produced in rats by partial sciatic nerve injury. Pain 1990;43(2):205-218.

[188] Shealy CN, Mortimer JT, Reswick JB. Electrical inhibition of pain by stimulation of the dorsal columns: preliminary clinical report. Anesth Analg 1967;46(4):489-491.

[189] Sheen K, Chung JM. Signs of neuropathic pain depend on signals from injured nerve fibers in a rat model. Brain Res 1993;610(1):62-68. 
[190] Shehab SA. Acute and chronic sectioning of fifth lumbar spinal nerve has equivalent effects on the primary afferents of sciatic nerve in rat spinal cord. J Comp Neurol 2009;517(4):481-492.

[191] Shields SD, Eckert WA, 3rd, Basbaum AI. Spared nerve injury model of neuropathic pain in the mouse: a behavioral and anatomic analysis. J Pain 2003;4(8):465-470.

[192] Sigtermans MJ, van Hilten JJ, Bauer MC, Arbous MS, Marinus J, Sarton EY, Dahan A. Ketamine produces effective and long-term pain relief in patients with Complex Regional Pain Syndrome Type 1. Pain 2009;145(3):304-311.

[193] Sindou MP, Mertens P, Bendavid U, Garcia-Larrea L, Mauguiere F. Predictive value of somatosensory evoked potentials for long-lasting pain relief after spinal cord stimulation: practical use for patient selection. Neurosurgery 2003;52(6):13741383; discussion 1383-1374.

[194] Sluka KA, Jordan HH, Willis WD, Westlund KN. Differential effects of N-methyl-Daspartate (NMDA) and non-NMDA receptor antagonists on spinal release of amino acids after development of acute arthritis in rats. Brain Res 1994;664(1-2):77-84.

[195] Smith SB, Crager SE, Mogil JS. Paclitaxel-induced neuropathic hypersensitivity in mice: responses in 10 inbred mouse strains. Life Sci 2004;74(21):2593-2604.

[196] Smits H, Kleef MV, Honig W, Gerver J, Gobrecht P, Joosten EA. Spinal cord stimulation induces c-Fos expression in the dorsal horn in rats with neuropathic pain after partial sciatic nerve injury. Neurosci Lett 2009;450(1):70-73.

[197] Smits H, Ultenius C, Deumens R, Koopmans GC, Honig WM, van Kleef $M$, Linderoth B, Joosten EA. Effect of spinal cord stimulation in an animal model of neuropathic pain relates to degree of tactile "allodynia". Neuroscience 2006;143(2):541-546.

[198] Sommer C, Kress M. Recent findings on how proinflammatory cytokines cause pain: peripheral mechanisms in inflammatory and neuropathic hyperalgesia. Neurosci Lett 2004;361(1-3):184-187.

[199] Sommer C, Schafers M. Painful mononeuropathy in C57BL/Wld mice with delayed wallerian degeneration: differential effects of cytokine production and nerve regeneration on thermal and mechanical hypersensitivity. Brain Res 1998;784(12):154-162. 
[200] Song XJ, Hu SJ, Greenquist KW, Zhang JM, LaMotte RH. Mechanical and thermal hyperalgesia and ectopic neuronal discharge after chronic compression of dorsal root ganglia. J Neurophysiol 1999;82(6):3347-3358.

[201] Song Z, Ultenius C, Meyerson BA, Linderoth B. Pain relief by spinal cord stimulation involves serotonergic mechanisms: an experimental study in a rat model of mononeuropathy. Pain 2009;147(1-3):241-248.

[202] Sprengel R, Single FN. Mice with genetically modified NMDA and AMPA receptors. Ann N Y Acad Sci 1999;868:494-501.

[203] Stanton-Hicks M. Complex regional pain syndrome: manifestations and the role of neurostimulation in its management. J Pain Symptom Manage 2006;31(4 Suppl):S20-24.

[204] Stanton-Hicks M, Baron R, Boas R, Gordh T, Harden N, Hendler N, Koltzenburg M, Raj P, Wilder R. Complex Regional Pain Syndromes: guidelines for therapy. Clin J Pain 1998;14(2):155-166.

[205] Stanton-Hicks MD, Burton AW, Bruehl SP, Carr DB, Harden RN, Hassenbusch SJ, Lubenow TR, Oakley JC, Racz GB, Raj PP, Rauck RL, Rezai AR. An updated interdisciplinary clinical pathway for CRPS: report of an expert panel. Pain Pract $2002 ; 2(1): 1-16$.

[206] Stiller CO, Cui JG, O'Connor WT, Brodin E, Meyerson BA, Linderoth B. Release of gamma-aminobutyric acid in the dorsal horn and suppression of tactile allodynia by spinal cord stimulation in mononeuropathic rats. Neurosurgery 1996;39(2):367374; discussion 374-365.

[207] Stubley LA, Martinez MA, Karmally S, Lopez T, Cejas P, Eaton MJ. Only early intervention with gamma-aminobutyric acid cell therapy is able to reverse neuropathic pain after partial nerve injury. J Neurotrauma 2001;18(4):471-477.

[208] Swett JE, Torigoe Y, Elie VR, Bourassa CM, Miller PG. Sensory neurons of the rat sciatic nerve. Exp Neurol 1991;114(1):82-103.

[209] Terayama R, Dubner R, Ren K. The roles of NMDA receptor activation and nucleus reticularis gigantocellularis in the time-dependent changes in descending inhibition after inflammation. Pain 2002;97(1-2):171-181. 
[210] Thomas MJ, Moody TD, Makhinson M, O'Dell TJ. Activity-dependent betaadrenergic modulation of low frequency stimulation induced LTP in the hippocampal CA1 region. Neuron 1996;17(3):475-482.

[211] Tian SL, Wang XY, Ding GH. Repeated electro-acupuncture attenuates chronic visceral hypersensitivity and spinal cord NMDA receptor phosphorylation in a rat irritable bowel syndrome model. Life Sci 2008;83(9-10):356-363.

[212] Toda K, Muneshige H, Asou T, Harada T, Okazaki M, Tachiki N, Horie N, Cheng W, Nakamura S. Basal blood flow in complex regional pain syndrome does not necessarily indicate vasoconstrictor nerve activity. Clin J Pain 2006;22(1):109-110.

[213] Todd AJ. Anatomy of primary afferents and projection neurones in the rat spinal dorsal horn with particular emphasis on substance $\mathrm{P}$ and the neurokinin 1 receptor. Exp Physiol 2002;87(2):245-249.

[214] Todd AJ, Sullivan AC. Light microscope study of the coexistence of GABA-like and glycine-like immunoreactivities in the spinal cord of the rat. J Comp Neurol 1990;296(3):496-505.

[215] Truin M, van Kleef M, Linderoth B, Smits H, Janssen SP, Joosten EA. Increased efficacy of early spinal cord stimulation in an animal model of neuropathic pain. Eur J Pain 2010.

[216] Truin M, van Kleef M, Verboeket Y, Deumens R, Honig W, Joosten EA. The effect of Spinal Cord Stimulation in mice with chronic neuropathic pain after partial ligation of the sciatic nerve. Pain 2009.

[217] Truin M, van Venrooij P, Duysens V, Deumens R, van Kleef M, Joosten EAJ. Spinal cord stimulation in a mouse chronic neuropathic pain model. Neuromodulation 2007;10(4):358-362.

[218] Turner JA, Hollingworth W, Comstock BA, Deyo RA. Spinal cord stimulation for failed back surgery syndrome: outcomes in a workers' compensation setting. Pain 2010;148(1):14-25.

[219] Uceyler N, Eberle T, Rolke R, Birklein F, Sommer C. Differential expression patterns of cytokines in complex regional pain syndrome. Pain 2007;132(1-2):195-205. 
[220] Ultenius C, Linderoth B, Meyerson BA, Wallin J. Spinal NMDA receptor phosphorylation correlates with the presence of neuropathic signs following peripheral nerve injury in the rat. Neurosci Lett 2006;399(1-2):85-90.

[221] van de Vusse AC, Stomp-van den Berg SG, Kessels AH, Weber WE. Randomised controlled trial of gabapentin in Complex Regional Pain Syndrome type 1 [ISRCTN84121379]. BMC Neurol 2004;4:13.

[222] van Eijs F, Smits H, Geurts JW, Kessels AG, Kemler MA, van Kleef M, Joosten EA, Faber CG. Brush-evoked allodynia predicts outcome of spinal cord stimulation in Complex Regional Pain Syndrome type 1. Eur J Pain 2009.

[223] Verdolin MH, Stedje-Larsen ET, Hickey AH. Ten consecutive cases of complex regional pain syndrome of less than 12 months duration in active duty United States military personnel treated with spinal cord stimulation. Anesth Analg 2007;104(6):1557-1560, table of contents.

[224] Visser E, Schug SA. The role of ketamine in pain management. Biomed Pharmacother 2006;60(7):341-348.

[225] Vogelaar CF, Vrinten DH, Hoekman MF, Brakkee JH, Burbach JP, Hamers FP. Sciatic nerve regeneration in mice and rats: recovery of sensory innervation is followed by a slowly retreating neuropathic pain-like syndrome. Brain Res 2004;1027(1-2):67-72.

[226] Vrinten DH, Hamers FF. 'CatWalk' automated quantitative gait analysis as a novel method to assess mechanical allodynia in the rat; a comparison with von Frey testing. Pain 2003;102(1-2):203-209.

[227] Wagner R, Janjigian M, Myers RR. Anti-inflammatory interleukin-10 therapy in CCI neuropathy decreases thermal hyperalgesia, macrophage recruitment, and endoneurial TNF-alpha expression. Pain 1998;74(1):35-42.

[228] Walker JL, Evans JM, Meade P, Resig P, Sisken BF. Gait-stance duration as a measure of injury and recovery in the rat sciatic nerve model. J Neurosci Methods 1994;52(1):47-52.

[229] Walker SM, Westin BD, Deumens R, Grafe M, Yaksh TL. Effects of intrathecal ketamine in the neonatal rat: evaluation of apoptosis and long-term functional outcome. Anesthesiology 2010;113(1):147-159. 
[230] Wall PD, Devor M, Inbal R, Scadding JW, Schonfeld D, Seltzer Z, Tomkiewicz MM. Autotomy following peripheral nerve lesions: experimental anaesthesia dolorosa. Pain 1979;7(2):103-111.

[231] Wallin J, Cui JG, Yakhnitsa V, Schechtmann G, Meyerson BA, Linderoth B. Gabapentin and pregabalin suppress tactile allodynia and potentiate spinal cord stimulation in a model of neuropathy. Eur J Pain 2002;6(4):261-272.

[232] Wallin J, Fiska A, Tjolsen A, Linderoth B, Hole K. Spinal cord stimulation inhibits long-term potentiation of spinal wide dynamic range neurons. Brain Res 2003;973(1):39-43.

[233] Wolf G, Gabay E, Tal M, Yirmiya R, Shavit Y. Genetic impairment of interleukin-1 signaling attenuates neuropathic pain, autotomy, and spontaneous ectopic neuronal activity, following nerve injury in mice. Pain 2006;120(3):315-324.

[234] Woolf CJ. Evidence for a central component of post-injury pain hypersensitivity. Nature 1983;306(5944):686-688.

[235] Woolf CJ, Mannion RJ. Neuropathic pain: aetiology, symptoms, mechanisms, and management. Lancet 1999;353(9168):1959-1964.

[236] Woolf CJ, Salter MW. Neuronal plasticity: increasing the gain in pain. Science 2000;288(5472):1765-1769.

[237] Woolf CJ, Thompson SW. The induction and maintenance of central sensitization is dependent on $\mathrm{N}$-methyl-D-aspartic acid receptor activation; implications for the treatment of post-injury pain hypersensitivity states. Pain 1991;44(3):293-299.

[238] Wu G, Ringkamp M, Murinson BB, Pogatzki EM, Hartke TV, Weerahandi HM, Campbell JN, Griffin JW, Meyer RA. Degeneration of myelinated efferent fibers induces spontaneous activity in uninjured C-fiber afferents. J Neurosci 2002;22(17):7746-7753.

[239] Xing GG, Liu FY, Qu XX, Han JS, Wan Y. Long-term synaptic plasticity in the spinal dorsal horn and its modulation by electroacupuncture in rats with neuropathic pain. Exp Neurol 2007;208(2):323-332.

[240] Xu JT, Xin WJ, Wei XH, Wu CY, Ge YX, Liu YL, Zang Y, Zhang T, Li YY, Liu XG. p38 activation in uninjured primary afferent neurons and in spinal microglia 
contributes to the development of neuropathic pain induced by selective motor fiber injury. Exp Neurol 2007;204(1):355-365.

[241] Xu JT, Xin WJ, Zang Y, Wu CY, Liu XG. The role of tumor necrosis factor-alpha in the neuropathic pain induced by Lumbar 5 ventral root transection in rat. Pain 2006;123(3):306-321.

[242] Yakhnitsa V, Linderoth B, Meyerson BA. Spinal cord stimulation attenuates dorsal horn neuronal hyperexcitability in a rat model of mononeuropathy. Pain 1999;79(23):223-233.

[243] Yamamoto T, Yaksh TL. Spinal pharmacology of thermal hyperesthesia induced by constriction injury of sciatic nerve. Excitatory amino acid antagonists. Pain 1992;49(1):121-128.

[244] Yoon YW, Na HS, Chung JM. Contributions of injured and intact afferents to neuropathic pain in an experimental rat model. Pain 1996;64(1):27-36.

[245] Zhou ZS, Zhao ZQ. Ketamine blockage of both tetrodotoxin (TTX)-sensitive and TTX-resistant sodium channels of rat dorsal root ganglion neurons. Brain Res Bull 2000;52(5):427-433.

[246] Zou X, Lin Q, Willis WD. Role of protein kinase A in phosphorylation of NMDA receptor 1 subunits in dorsal horn and spinothalamic tract neurons after intradermal injection of capsaicin in rats. Neuroscience 2002;115(3):775-786.

[247] Zou X, Lin Q, Willis WD. Effect of protein kinase C blockade on phosphorylation of NR1 in dorsal horn and spinothalamic tract cells caused by intradermal capsaicin injection in rats. Brain Res 2004;1020(1-2):95-105. 
The objectives of this thesis were to develop of a mouse model of neuropathic pain and spinal cord stimulation (SCS) and to increase the efficacy of spinal cord stimulation in an experimental animal model of neuropathic pain.

The first chapter gives an overview of the mechanisms underlying the development of neuropathic pain and spinal cord stimulation. It describes the efficacy of spinal cord stimulation on neuropathic pain in patients with complex regional pain syndrome and the need to increase this efficacy. Finally, the CatWalk is introduced as an alternative tool for the assessment of pain in experimental animal models.

The second chapter describes the behavioral effect of indirect inflammation of primary sensory neurons by a L5 ventral root transection in the C57BL/6 and 129] mouse. Using the CatWalk and von Frey monofilaments, our data showed no behavioral signs of neuropathic pain after the L 5 root transection in the different mouse strains.

The third chapter reports the development of a mouse (C57BL/6) model of neuropathic pain and SCS. The data showed that SCS in mice with neuropathic pain resulted in a robust attenuation of the neuropathic pain as measured with the von Frey monofilaments. It was also reported that all animals with neuropathic pain returned to baseline withdrawal thresholds after SCS.

The fourth chapter describes the inclusion of the CatWalk to measure behavioral changes in the mouse model described in chapter 3. Our result from chapter 3 could be reproduced using the von Frey monofilaments. The CatWalk parameters however did not show any changes after SCS.

The fifth chapter describes the behavioral effect of early SCS compared to late SCS in a rat model of neuropathic pain. The data showed that early SCS resulted in twice as much responders and an increased duration of the pain relieving effect as compared to late SCS.

The sixth chapter describes the combined use of ketamine and SCS in a rat model of neuropathic pain. The data reported that intrathecal ketamine is able to convert SCS non-responders into SCS responders. Furthermore, ketamine increases the duration of the pain relieving effect of SCS.

In chapter eight, the main findings of this thesis are summarized and discussed. In the first part, it is emphasized that experimental animal models of neuropathic pain (and SCS) cannot be automatically transferred from one specie to another (i.e. from rat to mouse), probably due to differences in anatomy and physiology. Furthermore, one has to be cautious in interpreting and correlating behavioral 
changes in animal models of neuropathic pain directly to "pain". In the second part, the different SCS strategies and the proposed underlying mechanisms are being discussed. 
Samenvatting 
De doelstellingen van dit proefschrift waren het ontwikkelen van een muismodel met neuropathische pijn en ruggenmergstimulatie en het verbeteren van de effectiviteit van ruggenmergstimulatie in een diermodel met neuropathische pijn.

Het eerste hoofdstuk geeft een overzicht van de mechanismen die verantwoordelijk zijn voor het ontstaan van neuropathische pijn en het effect van ruggenmergstimulatie. Het beschrijft de effectiviteit van ruggenmergstimulatie in het verminderen van neuropathische pijn bij patiënten met het complex regionaal pijn syndroom (CRPS) en dat deze effectiviteit nog verbeterd kan worden. Ook wordt de CatWalk geïntrodceerd als een alternatieve manier om pijn te meten in dieren met neuropathische pijn.

Het tweede hoofdstuk beschrijft de gedragsverandering ten gevolge van een indirecte ontsteking van primaire sensorische neuronen, na het doorsnijden van de voorste wortel van L5 in twee verschillende muizen stammen $(\mathrm{C} 57 / \mathrm{BL} / 6$ en 129J). Na het doorsnijden van de voorste wortel van L5 in deze twee muizenstammen werd er geen pijn gerelateerd gedrag gemeten met de CatWalk en de von Frey methode.

Het derde hoofdstuk doet verslag van de ontwikkeling van een muismodel (C57/BL/6) met neuropathische pijn en ruggenmergstimulatie. In dit model zorgt ruggenmergstimulatie voor een sterke vermindering van de neuropathische pijn, gemeten met de von Frey methode. Deze vermindering was zodanig dat alle muizen geen tekenen van pijn meer vertoonden.

Het vierde hoofdstuk beschrijft het gebruik van de CatWalk in het muismodel van hoofdstuk drie om pijn gerelateerd gedrag te meten. Gebruikmakend van de von Frey haren konden de resultaten van hoofdstuk 3 worden gereproduceerd. De CatWalk daarentegen kon geen verandering detecteren in gedrag na ruggenmerg stimulatie.

Het vijfde hoofdstuk beschrijft het effect van vroege ruggenmergstimulatie op pijn gerelateerd gedrag in de rat met neuropathische pijn. Het blijkt dat door vroege ruggenmergstimulatie twee keer zoveel ratten reageren (met pijnvermindering) op ruggenmergstimulatie in vergelijking met ratten die veel later ruggenmerg stimulatie krijgen. Daarnaast zorgt vroege ruggenmergstimulatie voor een langer pijnverminderend effect in vergelijking met late ruggenmergstimulatie.

Het zesde hoofdstuk gaat in op het effect van de combinatie van ketamine en ruggenmergstimulatie in de rat met neuropathische pijn. De ratten die eerst niet reageerden op ruggenmergstimulatie allen (non-responders) vertoonde na de combinatie van ruggenmergstimulatie en ketamine wel een vermindering van de 
pijn (het werden zo responders). Bovendien zorgde de toevoeging van ketamine voor een verlenging van het pijnverminderend effect van ruggenmergstimulatie.

In hoofdstuk acht worden alle bevindingen van dit proefschrift samengevat en bediscussieerd. Het eerste gedeelte benadrukt het feit dat bevindingen bij een dierexperimenteel model van neuropathische pijn en ruggenmergstimulatie, bijvoorbeeld in de rat, niet automatisch hetzelfde zullen zijn in bijvoorbeeld de muis. Dit komt vermoedelijk door verschillen in anatomie en fysiologie. Verder moet men voorzichtig zijn om gedragsveranderingen in een diermodel van neuropathische pijn meteen als "pijn" te interpreteren. In het tweede gedeelte komen de mogelijke mechanismen aan bod die het effect van ruggenmerg stimulatie kunnen verklaren. 
Co-authors \& affiliations 

Ronald Deumens, PhD

Dept. of Anesthesiology

University Hospital Maastricht, the Netherlands

Victor Duysens, BSc

Research and Technology

Medtronic Bakken Research Center, Maastricht

Wiel Honig

Dept. of Anesthesiology

Maastricht University, the Netherlands

Sofie P.M. Janssen, PhD

Dept. of Anesthesiology

University Hospital Maastricht, the Netherlands

Elbert A.J. Joosten, PhD

Dept. of Anesthesiology

University Hospital Maastricht, the Netherlands

Maastricht University, the Netherlands

Maarten van Kleef, $\mathrm{MD}, \mathrm{PhD}$

Dept. of Anesthesiology

University Hospital Maastricht, the Netherlands

Guido C. Koopmans, PhD

Spinal Cord Therapeutics GmbH

Erkrath, Germany

Bengt Linderoth, $\mathrm{MD}, \mathrm{PhD}$

Dept. of Clinical Neuroscience \& Section of Neurosurgery

Karolinska Institutet and University Hospital, Stockholm, Sweden

Eline Pracht, MD

Dept. of Anesthesiology

University Hospital Maastricht, the Netherlands

Helwin Smits, MD

Dept. of Anesthesiology

University Hospital Maastricht, the Netherlands

Paul van Venrooij,

Research and Technology

Medtronic Bakken Research Center, Maastricht

Yana Verboeket, MSc

University Hospital Maastricht, the Netherlands 

Dankwoord 

Het is dan zover... mijn proefschrift is klaar! Bij deze maak ik graag van de gelegenheid gebruik om de mensen te bedanken die dit allemaal mogelijk hebben gemaakt.

Om te beginnen wil ik mijn promotor, prof. dr. van Kleef bedanken. Om te beginnen natuurlijk voor de mogelijkheid om dit promotietraject te beginnen, maar ook voor het enthousiasme voor het onderzoek.

Dan natuurlijk ook mijn mentor dr. Joosten. Bert, wat heb je moeten afzien met mijn eigenwijsheid en mijn "mag ik even een korte vraag stellen". Maar je bleef altijd rustig. Bedankt voor een prachtige tijd in het lab, zowel op wetenschappelijk als persoonlijk vlak. Enorm bedankt dat je me de vrijheid gaf om met eigen ideeën te komen en dingen zelf uit te zoeken, maar altijd een oogje in het zeil hield om me wel "to the point" te houden.

De leden van de beoordelingscommissie, te weten voorzitter prof. dr. van Overbeeke, prof. dr. de Baets, prof. dr. van Hilten, dr. van Dongen en dr. Faber, wil ik van harte bedanken voor het beoordelen van dit proefschrift.

Prof. dr. Steinbusch wil ik bedanken voor het mede mogelijk maken van mijn promotietraject.

Ook dr. Deumens (Ron, Ronnie, double D), super bedankt. Jij was niet direct betrokken bij mijn onderzoek, maar je was nooit te beroerd (en dan bedoel ik ook echt nooit) om me bij te staan. Zonder jou was mijn laatste experiment in het water gevallen. Ook het enthousiasme en de humor die je in de groep bracht waren van onschatbare waarde.

Dan volgen nu de mensen met wie ik de meeste tijd heb doorgebracht, mijn kamergenoten. Robbie (of was het Robby), Rob......huuuuuuu. Wat hebben we veel plezier gehad! Stuiterbal, hotbox, Petronella zijn maar enkele steekwoorden die, nu ik dit schrijf, alweer een heel grote glimlach op mijn gezicht toveren. Ook op wetenschappelijk vlak heb je me vaak uit de brand geholpen $(1 \mathrm{ml}=\ldots \mu l$ ?). Heel veel succes met je nieuwe baan en natuurlijk heel veel geluk met Phien.

Anneke (boterham yourself), thank you for teaching me the right way to interpret the CatWalk data. Without you, it would still be just a bunch of numbers to me. Furthermore, I apologize for all the noise I made. Good luck in the future.

Helwin (Smitsie, klakklak), bedankt voor het aanleren van de Seltzer en de implantaties. Daarnaast heb jij het soort humor dat mijn dag helemaal goed kon maken. De woorden: populatiemanagement en diarree aan data zijn legendarisch geworden op de kamer. Succes met jouw promotie.

Liesbeth (heb helemaal geen bijnaam voor jou), ook met jou heb ik wat afgelachen. Maar ook heb je veel geduld getoond met mij bij het Western Blotten (Nr2B bestaat niet)! Als ik een vraag had met betrekking tot mechanismen kon je me altijd helpen. Tnx! Ook jij succes met het afronden van je promotietraject. Dan natuurlijk Wouter (daar is ie weer: werkelijk, kaWouter Plop). Wat hebben 
we veel over statistiek en de interpretatie van resultaten gediscussieerd! Bedankt voor alle inzichten. Succes in de kliniek en met het afronden van je boekje.

Sabien (appelsab), bedankt voor je vrolijkheid en de lol die we hebben gehad in Athene. Ook delen we dezelfde droom: hangmat op het werk. Je hebt nog een beengitaar van me tegoed! Veel plezier met je geitjes!

Sofie (Sofieke), bedankt voor alle hulp en humor het laatste jaar. Het aanleren van coupes snijden, kleuren en beoordelen was voor mij nieuw. Ook jouw tips bij mijn laatste manuscript waren erg waardevol. Veel plezier in jullie nieuwe huis.

Bart (wie...van Gorrup), ook jij bedankt voor de lol die we hebben gehad, maar ook dat je me hebt geleerd hoe de dieren te handelen (bartelen) wat enorm goed van pas is gekomen.

Joris, we hebben wat afgerookt (ondanks dat je gestopt was). Jouw ongecompliceerde blik op onderzoek heeft me vaak geholpen om een moeilijk probleem op te lossen. Bedankt hiervoor. Heel veel succes met het afronden van jouw proefschrift.

Geen kamergenoot, maar wel nauw betrokken bij mijn promotietraject: Joh, Wiel (Wieléke). Bedankt voor jouw hulp bij de meer praktische zaken die komen kijken bij het doen van onderzoek. Ook jouw hulp bij mijn eerste experiment was onmisbaar. Ik ga het kletsen over computers en nieuwsgroepen missen.

Marjan en Denise, bedankt voor jullie hulp bij het Western Blotten.

Ook wil ik mijn studenten Yana (nee, de CatWalk maak je niet schoon met een schuursponsje) en Lieve bedanken voor hun inbreng en inzet. Het was leuk om jullie iets te leren.

Paul en Victor, enorm bedankt voor jullie vakkundige en snelle werk. Ik hoefde maar te bellen en binnen 2 weken werden de electroden persoonlijk langs gebracht (wat een service)!

Dan zijn er ook nog de mensen die me hebben gesteund, buiten het werk om.

Tim en Aggie, als ik mezelf ter adoptie zou opgeven, zou ik willen dat jullie mij adopteren. In zekere zin hebben jullie dat ook gedaan. Al vanaf het begin ben ik "kind aan huis" bij jullie. Door al het plezier, de etentjes (beenham rules), de wijntjes (Pineaud rules meer), maar ook de discussies over onderzoek hebben het mij allemaal een stuk makkelijker gemaakt. Aggie, door jouw vrolijke drukte en ondernemende geest was er altijd wat te beleven met jou. Over jouw achternaam worden het we het nog wel eens. Lubje, bedankt voor alle jaren van steun, lol en liefde.

Woutje, bedankt dat je altijd bleef bellen. Jij was er vanaf het eerste uur bij. Ondanks dat je in Eindhoven zit heb je me altijd weten op te vrolijken met jouw geniale humor. Tnx voor alles!

Juur en Mar (en tegenwoordig ook Philomijn), jullie hebben niks met onderzoek, maar wilden altijd precies weten waar ik mee bezig was. Daarnaast wisten jullie mij altijd weer energie te geven na een weekendje Amsterdam. Ik hou van jullie. 
Bousie, bedankt voor het leren banden plakken. Ik heb er veel profijt van gehad.

Rik, Thieu en Maarten. Tnx voor alle mooie momenten in Amsterdam en Alanya.

Yvonne, bedankt dat je me de ruimte gaf om aan mijn boekje te werken.

Ook wil ik Marc, Jurren, Morten, Jet, Marieke, Jerome en Roel bedanken. Zij hebben mij het enthousiasme voor het onderzoek bijgebracht.

Piet, Gerry, Michiel, Jeroen en Linda, bedankt voor alles. Ook al weet ik niks van voetbal, toch mag ik bij jullie over de vloer komen. Nu ik wat meer tijd heb, hoop ik dat de bezoekjes aan Rijkevoort, Deventer en Den Haag in frequentie zullen toenemen.

Lieve papsie en mamsie, bedankt voor alles wat jullie voor mij hebben gedaan. Het is heerlijk om te weten dat ik altijd kan rekenen op jullie liefde, plezier en advies. Super bedankt voor jullie steun in de keuzes die ik heb gemaakt. Ik hou van jullie!

Janneke, superbabe, bedankt voor al je geduld, hulp en steun. Jij kan mij altijd laten lachen. Zonder jou was het allemaal veel minder makkelijk geweest. Tnx lief! 

Curriculum Vitae 

Michiel Truin was born on February 5, 1977, in Nijmegen, the Netherlands. After graduation he studied Health Sciences at Maastricht University for two years. In 1999, he started to study Medicine at Maastricht University, which he finished in 2005. In 2006, he started his AGIKO project at the Academic Hospital Maastricht (AZM) for the department of anaesthesiology. After 1 year of research he worked in the hospital for 1.5 years as an intern anaesthesiology. After this, he returned to finish his PhD project at Maastricht University.

His PhD project was aimed at developing animal models of neuropathic pain and spinal cord stimulation. Using these models, the possibility of increasing the efficacy of spinal cord stimulation was explored.

$\mathrm{He}$ is currently following his General Practitioner training at Maastricht University. 

Publications 



\section{Papers}

Truin M, Koopmans G, Pragt E, van Kleef M, Joosten E.A. Sensitzed primary afferent related behavioral changes in mice with $L 5$ ventral root transection. (submitted)

Truin M, Janssen S.P.M., van Kleef M, Joosten E.A. Successful pain relief in nonresponders to spinal cord stimulation: the combined use of ketamine and spinal cord stimulation. European Journal of Pain 2011, Epub ahead of print.

Janssen S.P.M., Truin M, M. van Kleef, E.A. Joosten. Differential gabaergic disinhibition during the development of painful peripheral neuropathy. Neuroscience 2011, Epub ahead of print.

Truin $M$, van Kleef $M$, Linderoth B, Smits $H$, Janssen $S$, Joosten $E$. Increased efficacy of early spinal cord stimulation in an animal model of neuropathic pain. European Journal of Pain 2010, 15 (2): 111-7.

Smits H, Truin M, van Kleef M, Joosten E.A, Janssen S. Spinal cord stimulation in the treatment of neuropathic pain: from experimental findings towards clinical understanding. Nederlands Tijdschrift voor anesthesiologie 2009; 21(2): 10-3.

Truin M, van Kleef M, Verboeket $Y$, Deumens R, Honig W, Joosten E.A. The effect of spinal cord stimulation in mice with chronic neuropathic pain after partial ligation of the sciatic nerve. Pain 2009; 145(3):312-8.

Truin M, van Venrooij P, Duysens V, Deumens R, van Kleef M, Joosten E.A. Spinal cord stimulation in a mouse chronic neuropathic pain model. Neuromodulation 2007;10(4):358-362.

Truin M, Thomsen M.B, van Opstal J.M, Beekman J.D.M, Volders P.G.A, Stengl $M$, Vos M.A. Sudden cardiac death in dogs with remodeled hearts is associated with larger beat-to-beat variability of repolarization. Basic Research in Cardiology 2005;100(3):279-287. 


\section{Presentations}

Truin M, Janssen S.P.M, van Kleef M, S.P.M., Joosten. Effect of Spinal Cord Stimulation on tactile hypersensitivity is potentiated by a sub-effective dose of Ketamine in an animal model of chronic neuropathic pain. $7^{\mathrm{e}}$ Wetenschapsdag Anesthesiologie, September 24, 2010, Ede, The Netherlands. Oral presentation

Truin M, van Kleef, Jaken R, Janssen S.P.M., Joosten E.A. Early spinal cord stimulation results in a better outcome compared to late treatment in an animal model of neuropathic pain. Third International Congress on neuropathic pain, May, 27-30, 2010, Athens, Greece. Poster presentation.

Truin M. Neuromodulatie (spinal cord stimulation) in de muis met chronische neuropathische pijn. Wetenschappelijke dag Vereniging voor Neuromodulatie Nederland, Novermber 12, 2009, Amersfoort, The Netherlands. Oral presentation.

Truin M, Verboeket $Y$, Honig W, Deumens R, van Kleef M, Joosten E.A. The dregree of neuropathic pain is mice with chronic neuropatic pain does not relate to the response to spinal cord stimulation. World Pain Congress, May 13-16, 2008, New York, USA. Poster presentation.

Truin M, Verboeket Y, Honig W, Deumens R, van Kleef M, Joosten E.A. Pain indiced gait adaptations after chronic neuropathic pain and spinal cord stimulation. $5^{\text {e }}$ Wetenschapsdag Anesthesiologie, September 25, 2008, Utrecht, The Netherlands. Oral presentation.

Truin M, Verboeket Y, Honig W, Deumens R, van Kleef M, Joosten E.A. Effect of spinal cord stimulation is not related to the degree of mechanical allodynia in a mouse model of neuopathic pain. Society for Neuroscience, November 15-19, 2008, Washington, DC, USA. Poster presentation.

Truin M, Verboeket Y, Honig W, Deumens R, van Kleef M, Joosten E.A. Spinal cord stimulation in a mouse chronic neuropathic pain model. Second International Congress on neuropathic pain, June 7-10, 2007, Berlin, Germany. Poster presentation.

Truin M, Verboeket Y, Honig W, Deumens R, Joosten E.A, van Kleef M. Spinal cord stimulatie als behandeling van chronische neuropathische pijn in de muis. Anesthesiologendagen, May 10-1, 2007, Maastricht, The Netherlands. Oral presentation. 



